\title{
Iridium-Catalyzed Enantioconvergent Allylation of a Boron-Stabilized Organozinc Reagent
}

Panchi Guo, and Miao Zhan*

Institute of Medical Research, Northwestern Polytechnical University, Xi'an Shaanxi, 710072, P.R.China.

Email: mzhan@nwpu.edu.cn

\section{Table of Contents}

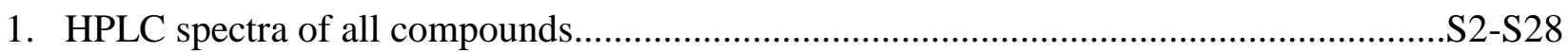

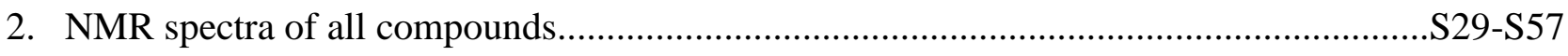



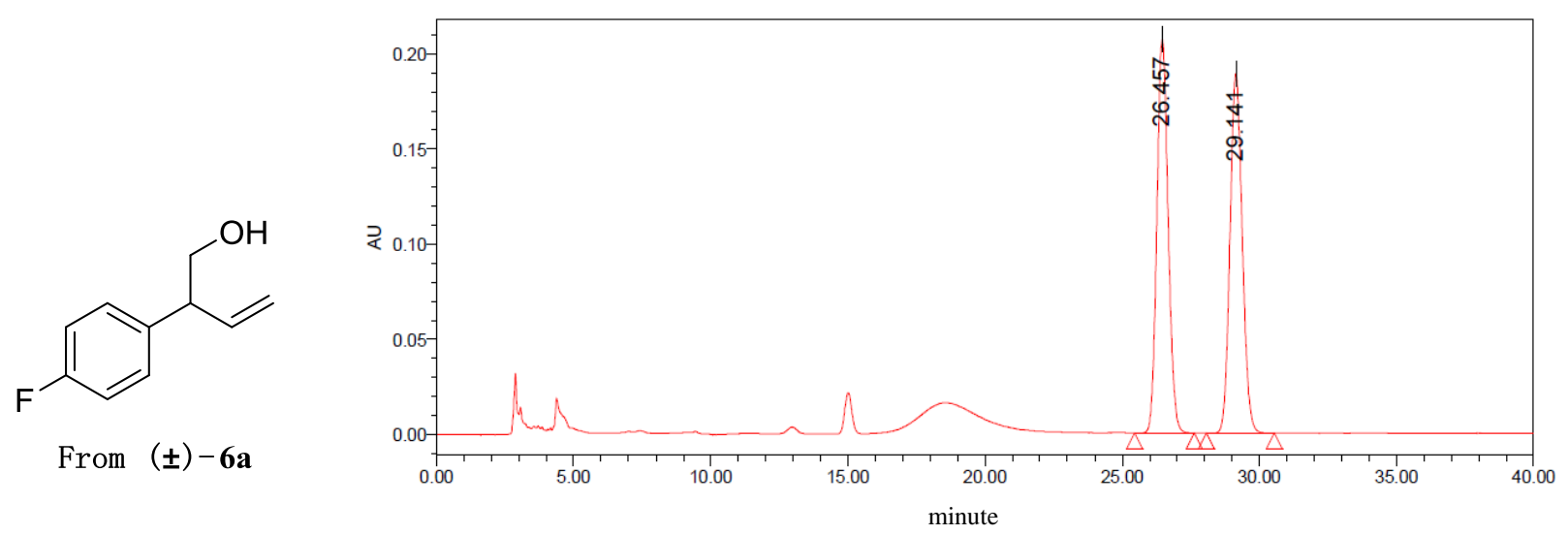

\begin{tabular}{cccc}
\hline Peak & Retention time & Area & Area\% \\
\hline 1 & 26.457 & 5884911 & 49.97 \\
2 & 29.141 & 5891788 & 50.03 \\
\hline
\end{tabular}

$210 \mathrm{~nm}$ Detection

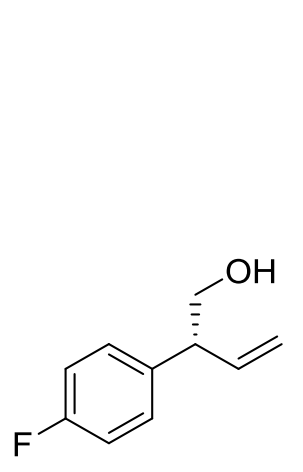

From 6a

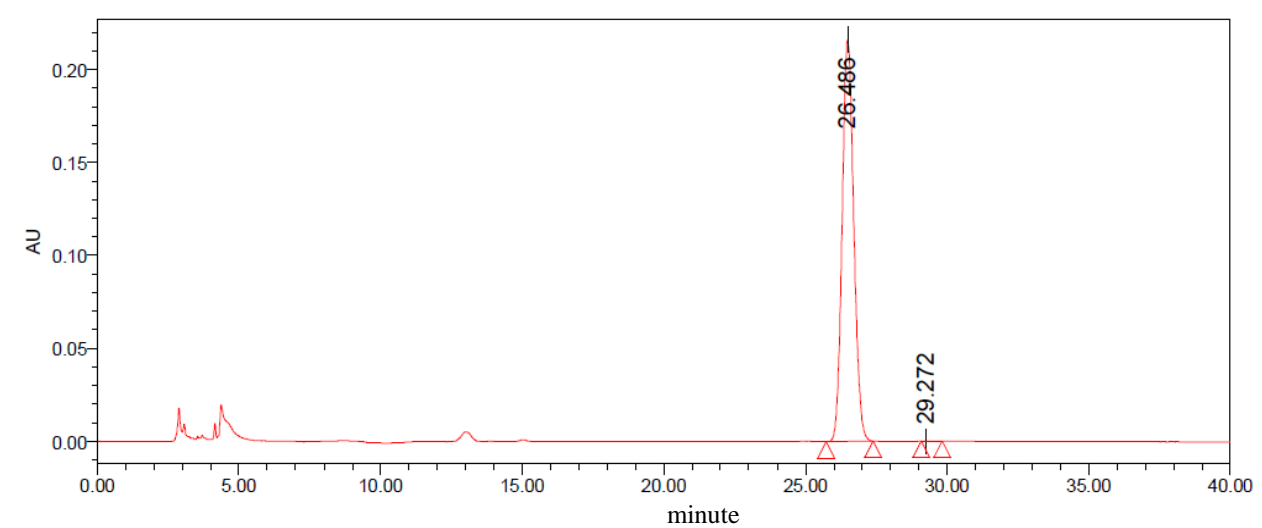

\begin{tabular}{cccc}
\hline Peak & Retention time & Area & Area\% \\
\hline 1 & 26.486 & 6168134 & 99.98 \\
2 & 29.272 & 1082 & 0.02 \\
\hline
\end{tabular}

$210 \mathrm{~nm}$ Detection 


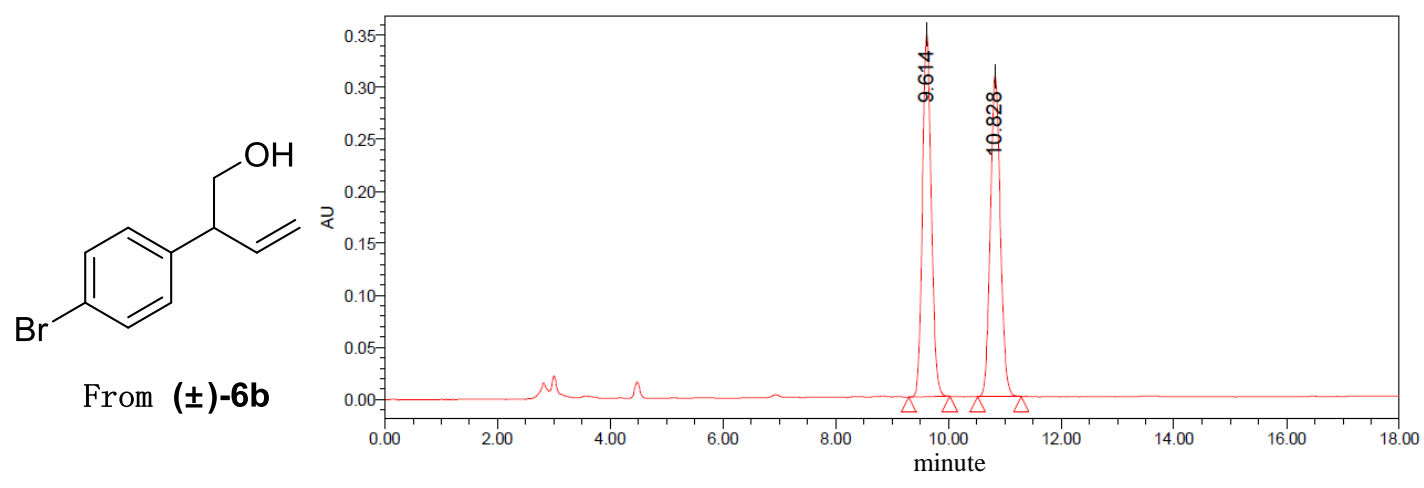

\begin{tabular}{cccc}
\hline Peak & Retention time & Area & Area\% \\
\hline 1 & 9.614 & 3812994 & 50.01 \\
2 & 10.828 & 3810797 & 49.99 \\
\hline
\end{tabular}

$210 \mathrm{~nm}$ Detection

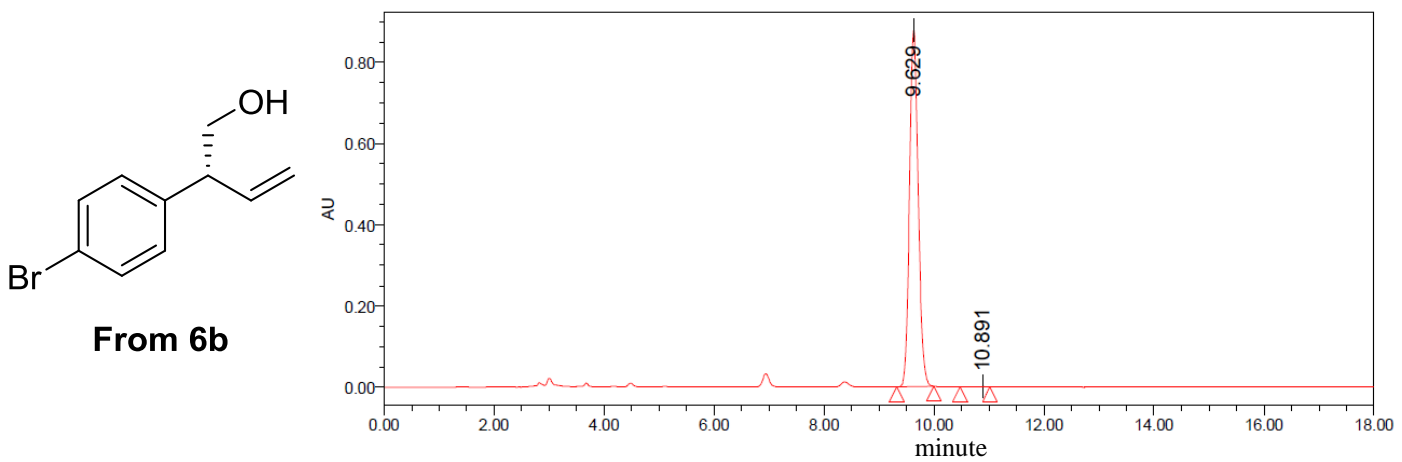

\begin{tabular}{cccc}
\hline Peak & Retention time & Area & Area\% \\
\hline 1 & 9.629 & 9675031 & 99.94 \\
2 & 10.891 & 5846 & 0.06 \\
\hline
\end{tabular}

$210 \mathrm{~nm}$ Detection 


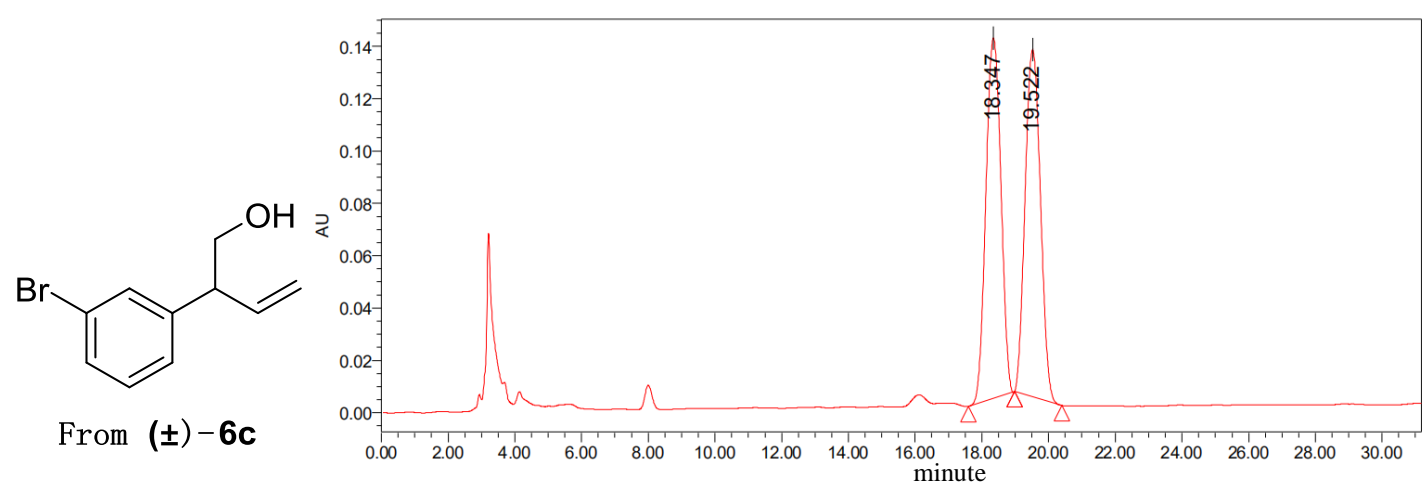

\begin{tabular}{cccc}
\hline Peak & Retention time & Area & Area\% \\
\hline 1 & 18.347 & 4299630 & 50.35 \\
2 & 19.522 & 4239939 & 49.65 \\
\hline
\end{tabular}

$210 \mathrm{~nm}$ Detection

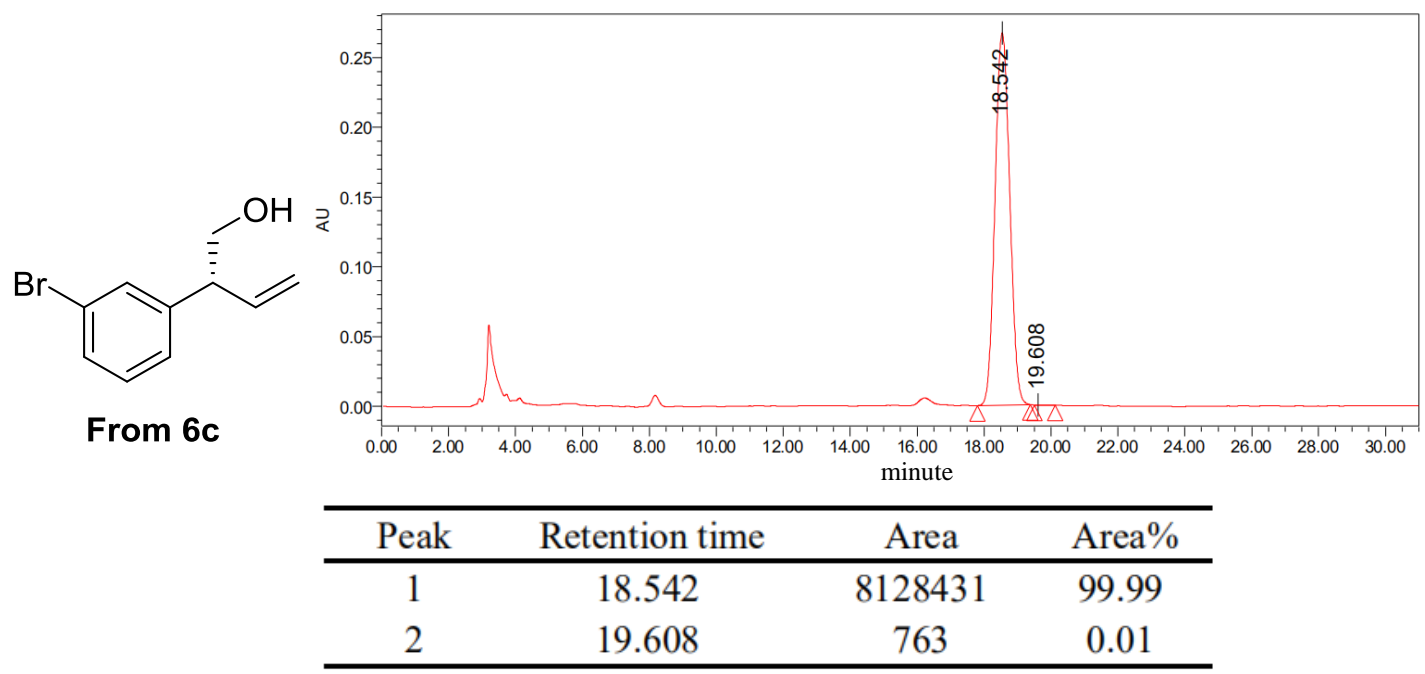

$210 \mathrm{~nm}$ Detection 
<smiles>C=CC(CO)c1ccc(Cl)cc1</smiles>

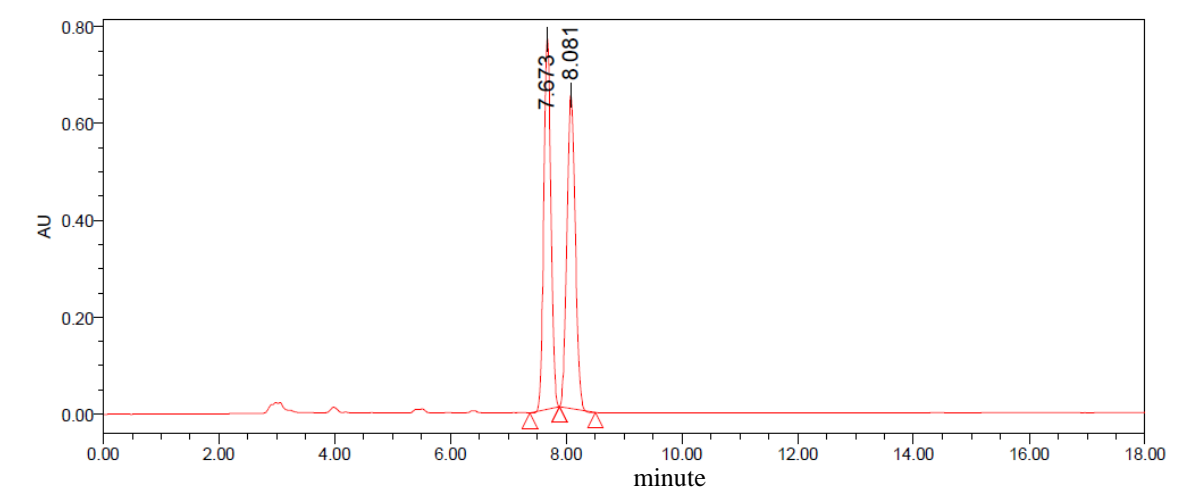

\begin{tabular}{cccc}
\hline Peak & Retention time & Area & Area\% \\
\hline 1 & 7.673 & 6544764 & 50.09 \\
2 & 8.081 & 6521056 & 49.91 \\
\hline
\end{tabular}

\section{$210 \mathrm{~nm}$ Detection}<smiles>C=C[C@H](CO)c1ccc(Cl)cc1</smiles>

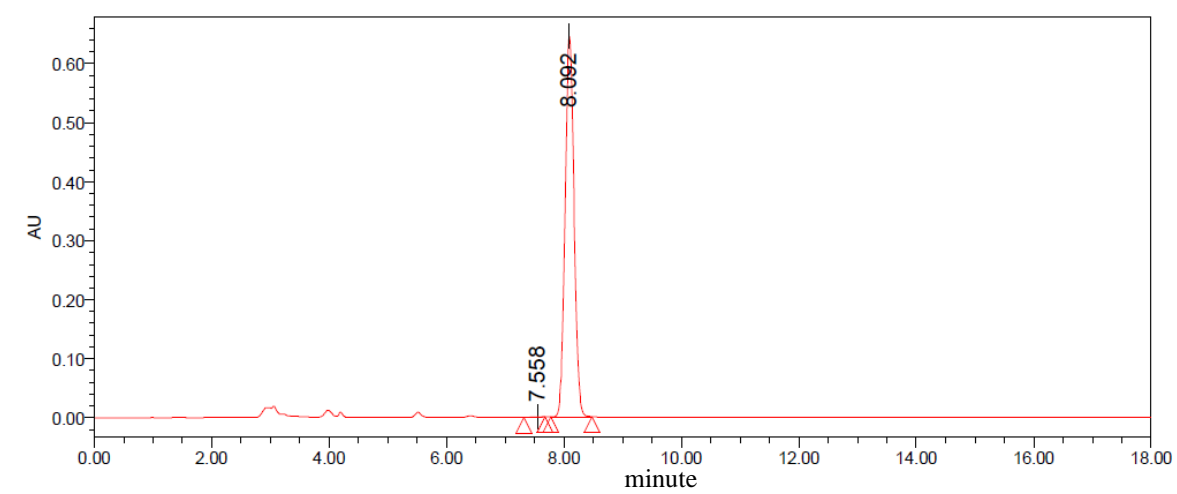

\begin{tabular}{cccc}
\hline Peak & Retention time & Area & Area\% \\
\hline 1 & 7.558 & 5194 & 0.08 \\
2 & 8.092 & 6847873 & 99.92 \\
\hline
\end{tabular}

$210 \mathrm{~nm}$ Detection 


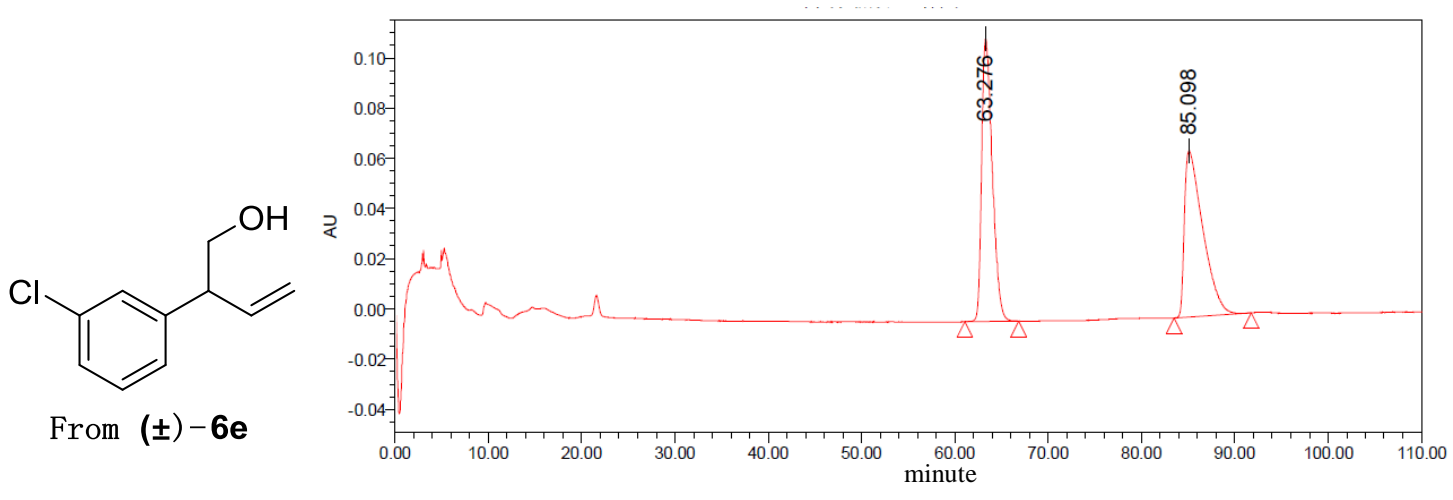

\begin{tabular}{cccc}
\hline Peak & Retention time & Area & Area\% \\
\hline 1 & 63.276 & 9010310 & 50.06 \\
2 & 85.098 & 8990507 & 49.94 \\
\hline
\end{tabular}

$210 \mathrm{~nm}$ Detection

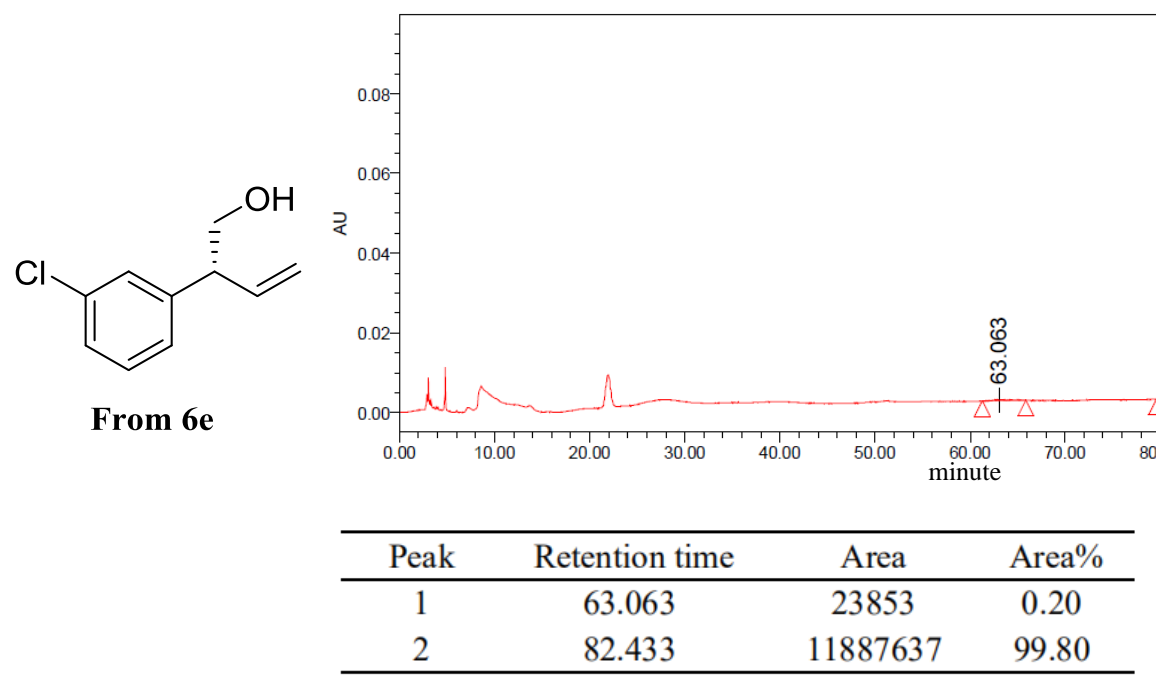

$210 \mathrm{~nm}$ Detection 


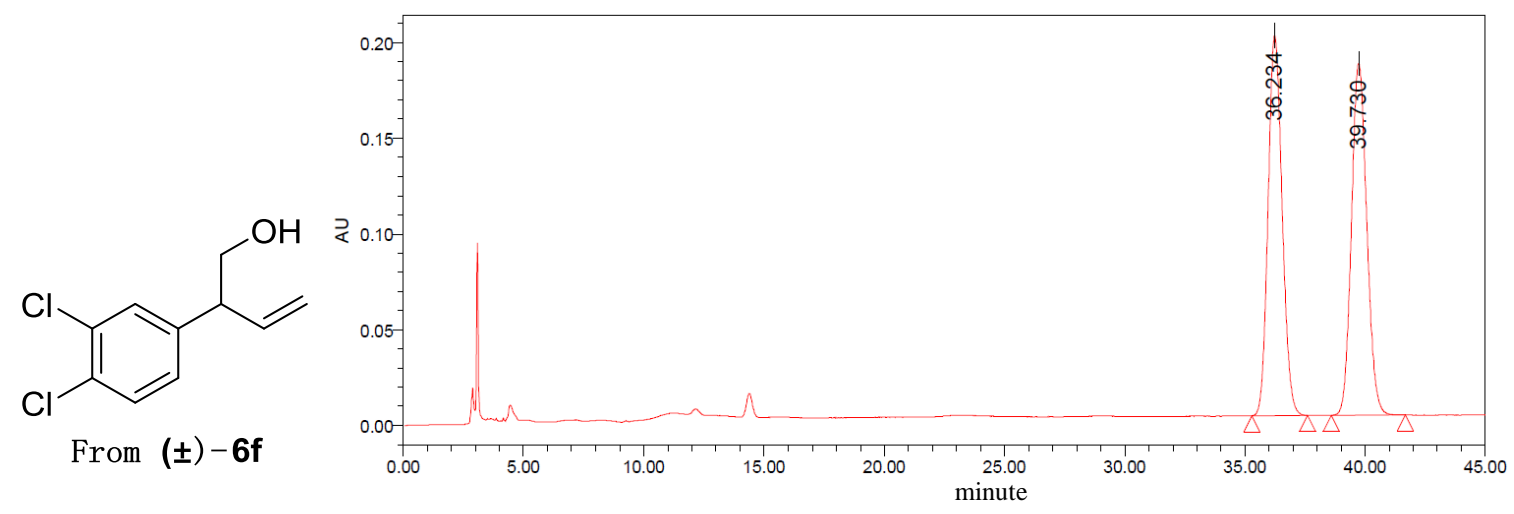

\begin{tabular}{cccc}
\hline Peak & Retention time & Area & Area\% \\
\hline 1 & 36.234 & 8083595 & 49.88 \\
2 & 39.730 & 8122478 & 50.12 \\
\hline
\end{tabular}

$210 \mathrm{~nm}$ Detection

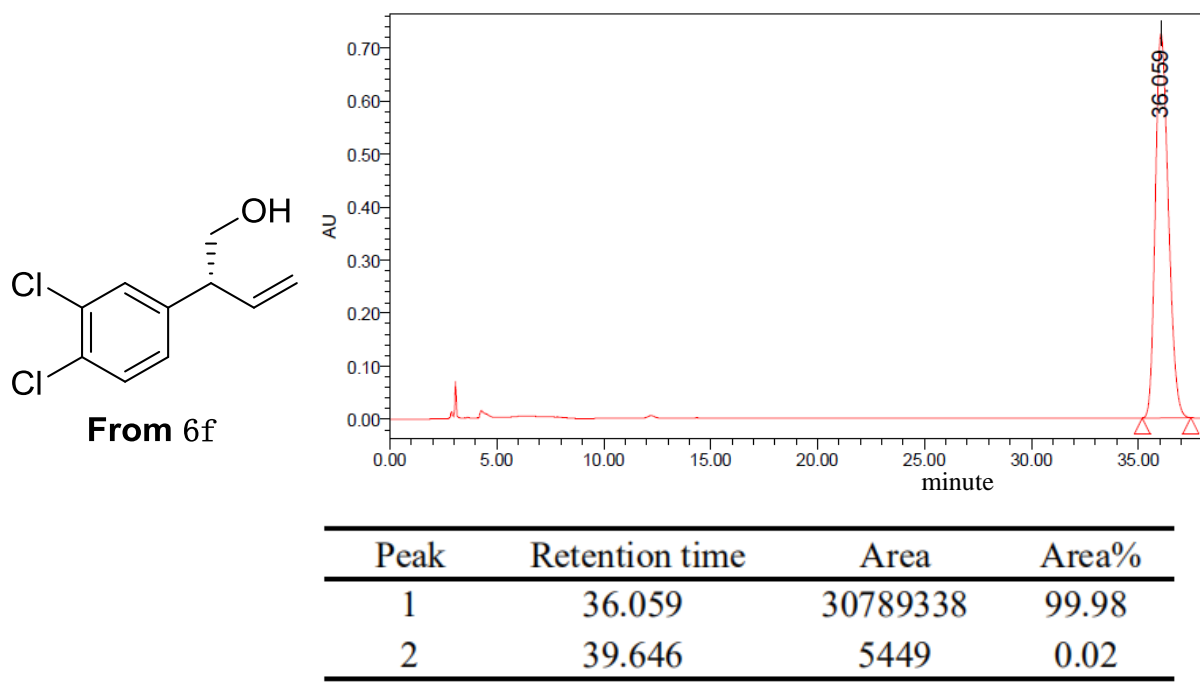

$210 \mathrm{~nm}$ Detection 


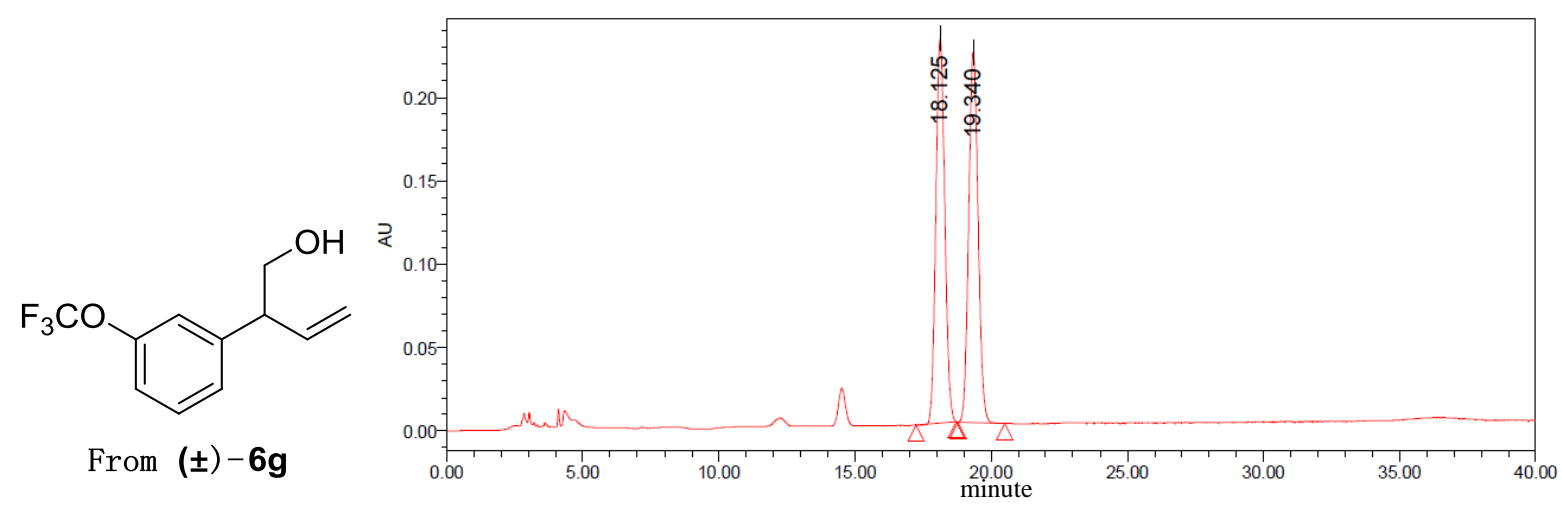

\begin{tabular}{cccc}
\hline Peak & Retention time & Area & Area $\%$ \\
\hline 1 & 18.125 & 5263908 & 49.70 \\
2 & 19.340 & 5328406 & 50.30 \\
\hline
\end{tabular}

$210 \mathrm{~nm}$ Detection

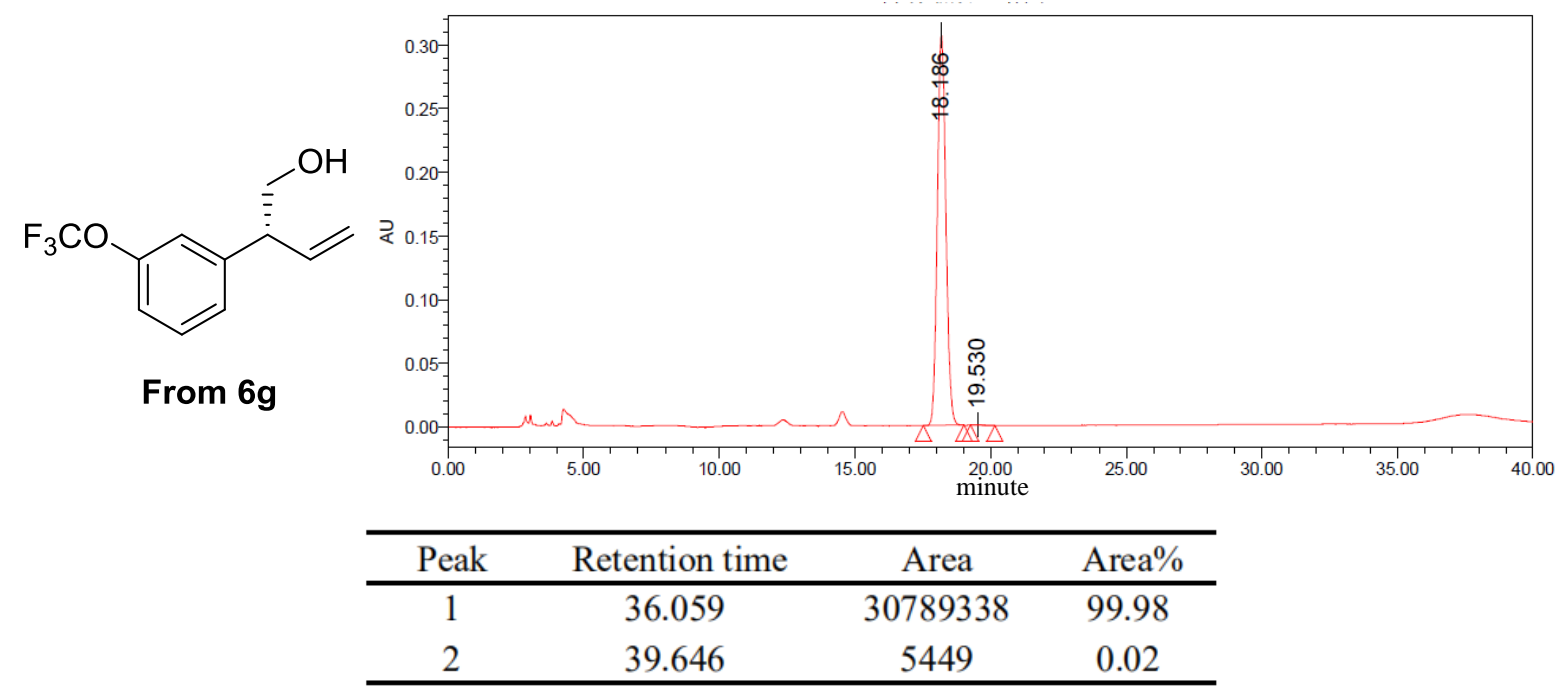

$210 \mathrm{~nm}$ Detection 


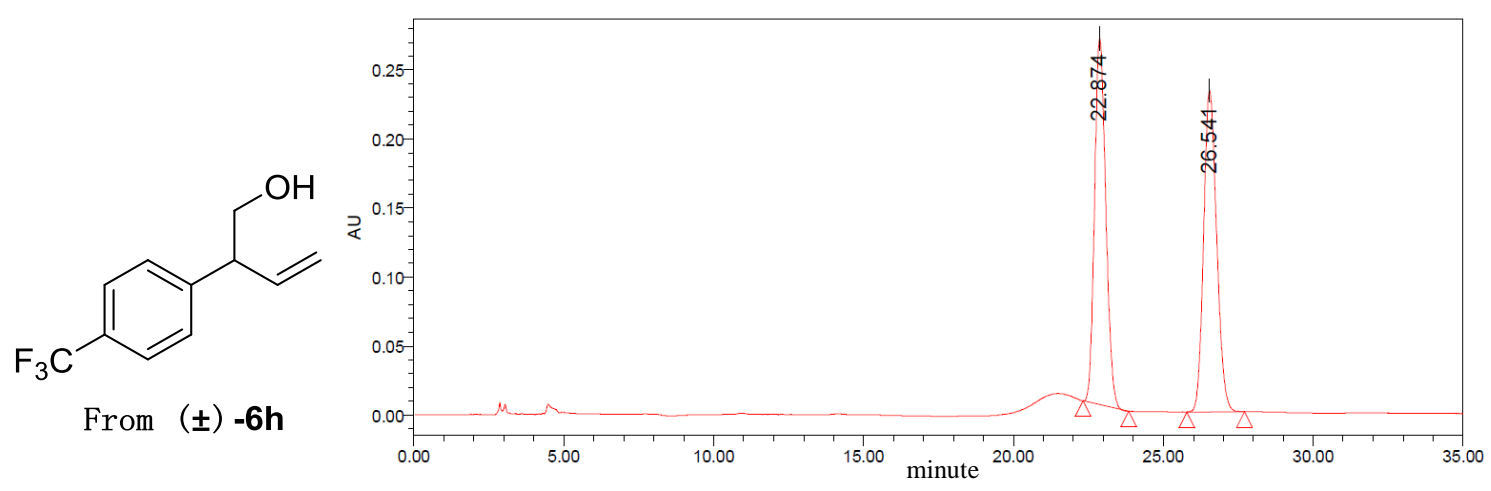

\begin{tabular}{cccc}
\hline Peak & Retention time & Area & Area\% \\
\hline 1 & 22.874 & 6925602 & 49.55 \\
2 & 26.541 & 7051434 & 50.45 \\
\hline
\end{tabular}

$210 \mathrm{~nm}$ Detection

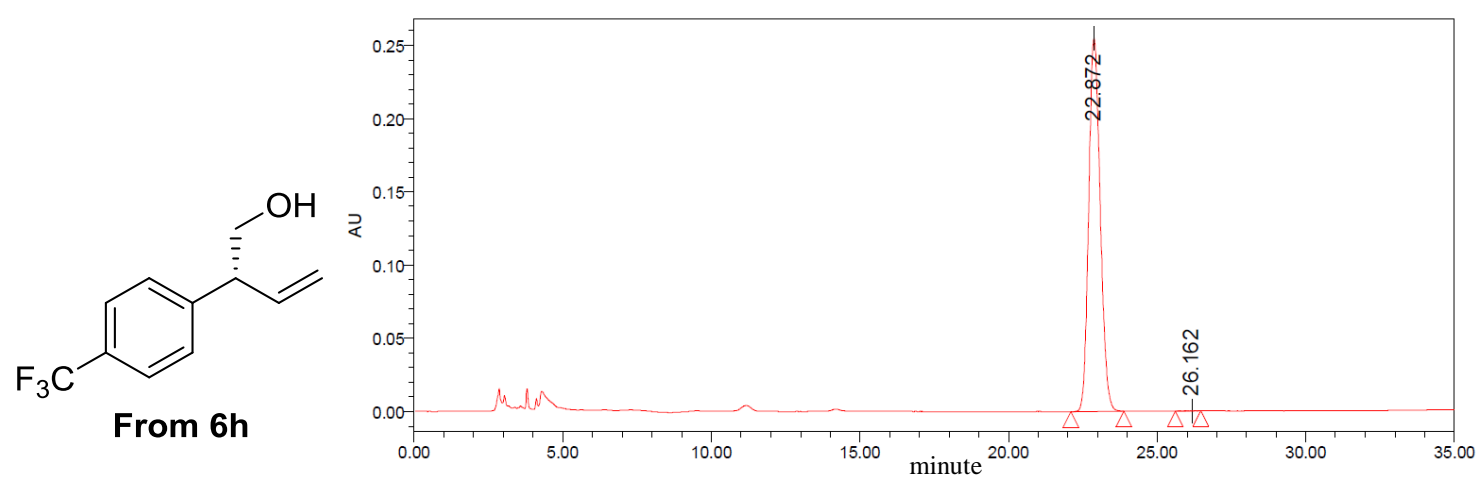

\begin{tabular}{cccc}
\hline Peak & Retention time & Area & Area\% \\
\hline 1 & 22.872 & 6860716 & 99.99 \\
2 & 26.162 & 348 & 0.01 \\
\hline
\end{tabular}

$210 \mathrm{~nm}$ Detection 


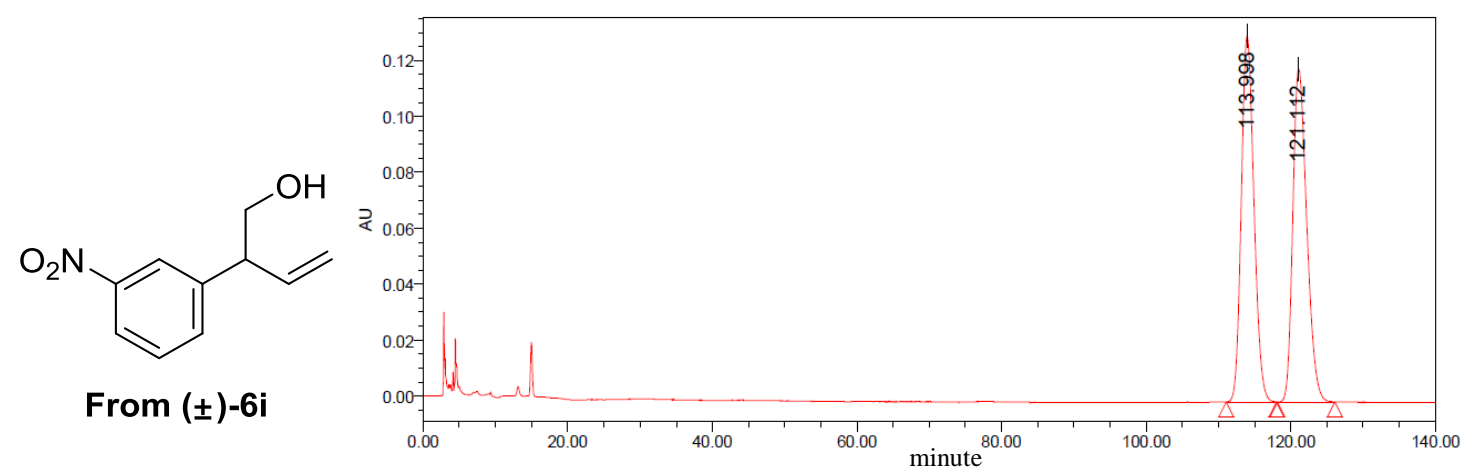

\begin{tabular}{cccc}
\hline Peak & Retention time & Area & Area\% \\
\hline 1 & 113.998 & 15877537 & 49.98 \\
2 & 121.112 & 15890329 & 50.02 \\
\hline
\end{tabular}

$210 \mathrm{~nm}$ Detection

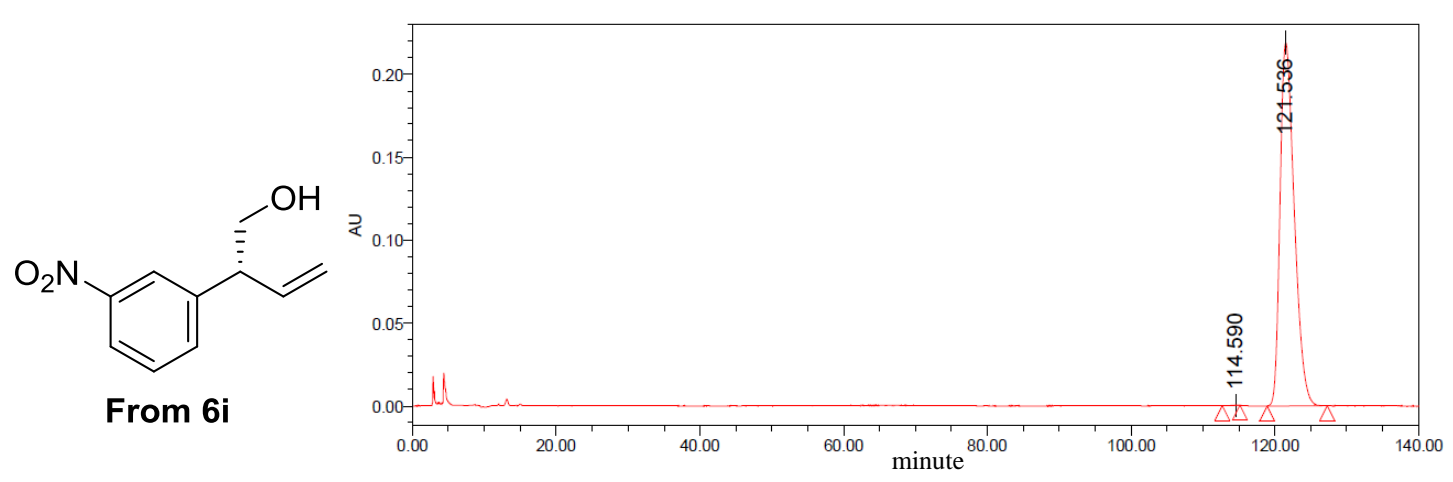

\begin{tabular}{cccc}
\hline Peak & Retention time & Area & Area $\%$ \\
\hline 1 & 114.590 & 6544 & 0.02 \\
2 & 121.536 & 30030553 & 99.98 \\
\hline
\end{tabular}

$210 \mathrm{~nm}$ Detection 


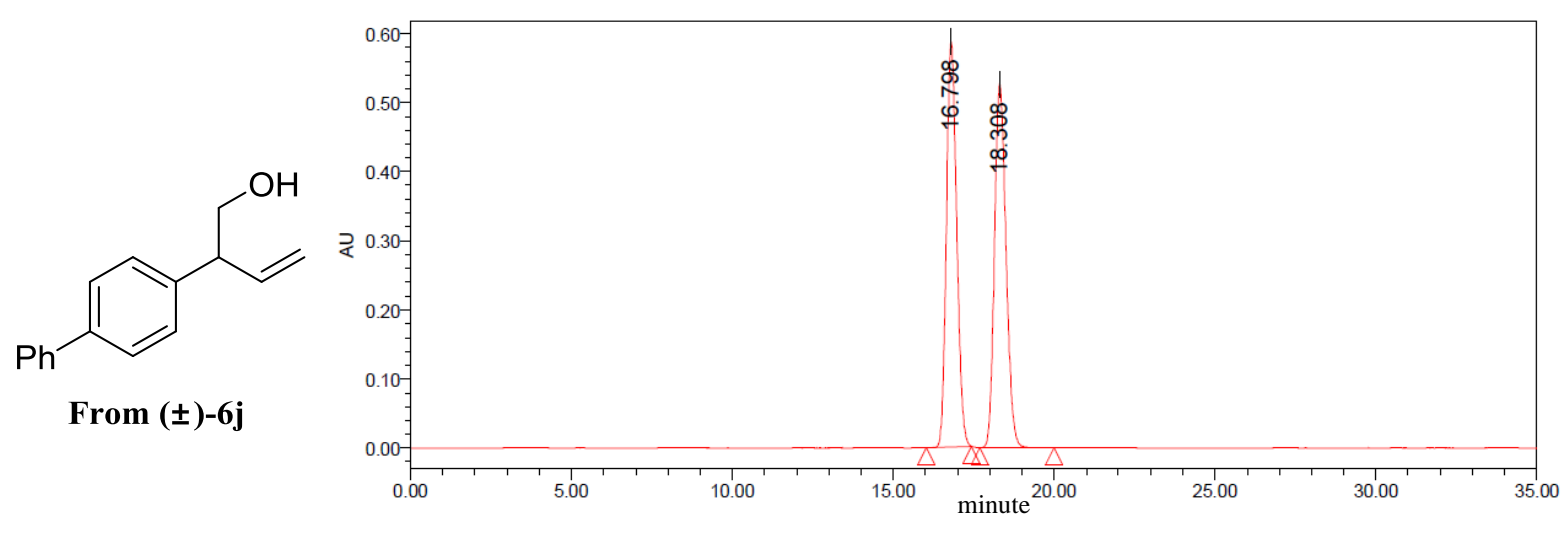

\begin{tabular}{cccc}
\hline Peak & Retention time & Area & Area\% \\
\hline 1 & 16.798 & 12991540 & 49.81 \\
2 & 18.308 & 13090520 & 50.19 \\
\hline
\end{tabular}

$254 \mathrm{~nm}$ Detection

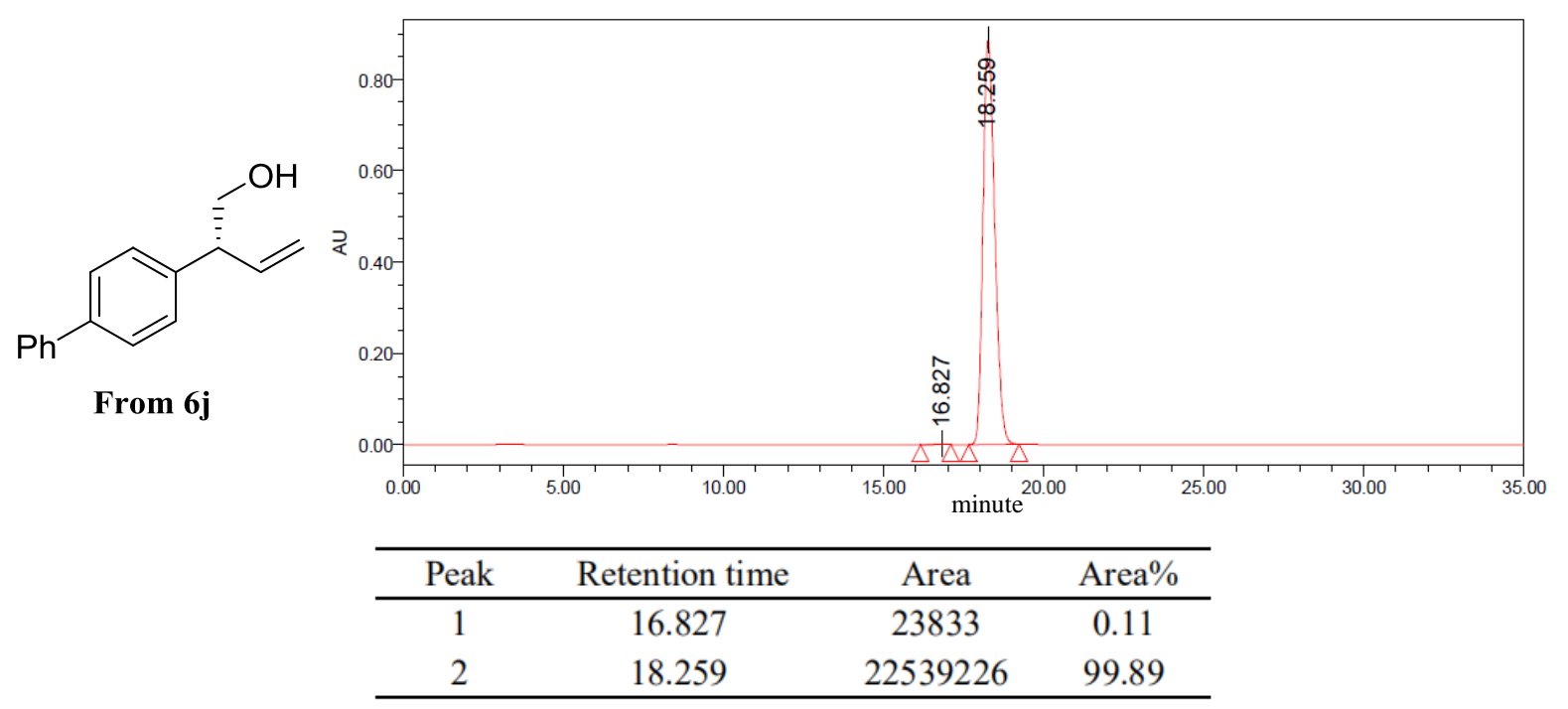

$254 \mathrm{~nm}$ Detection 


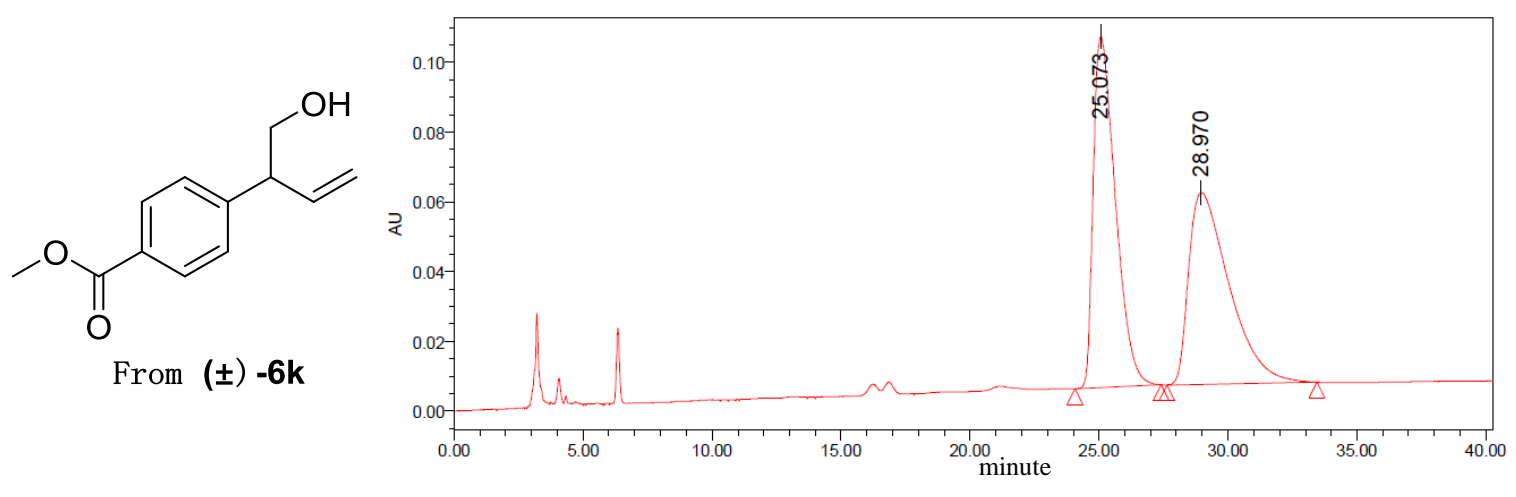

\begin{tabular}{cccc}
\hline Peak & Retention time & Area & Area\% \\
\hline 1 & 25.073 & 5995143 & 49.99 \\
2 & 28.970 & 5998077 & 50.01 \\
\hline
\end{tabular}

$210 \mathrm{~nm}$ Detection

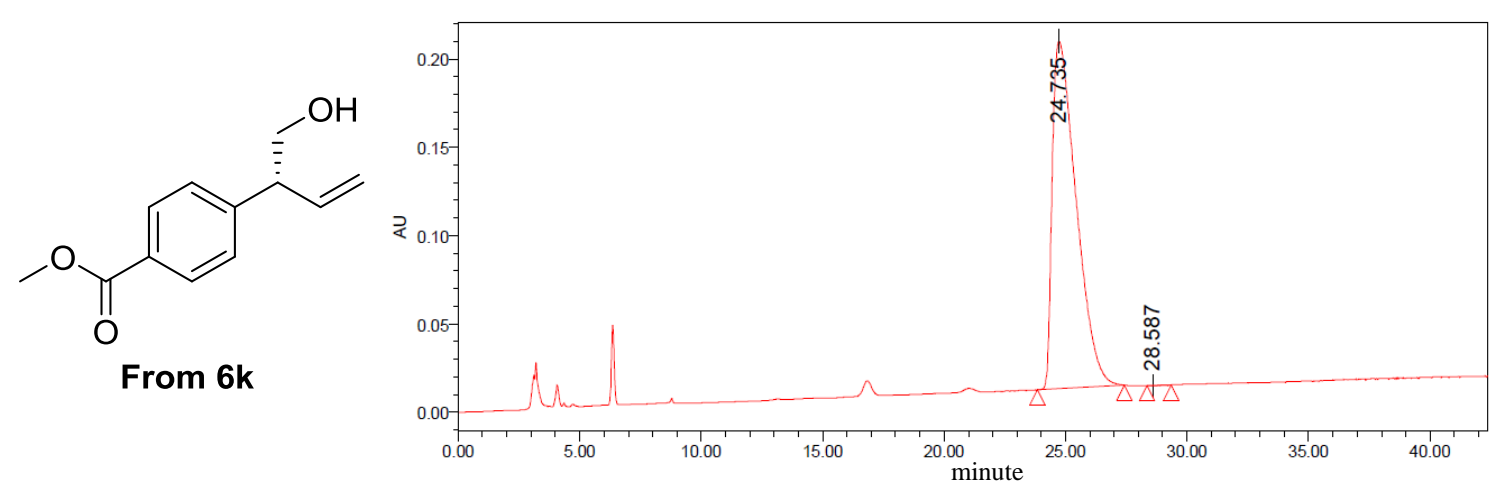

\begin{tabular}{cccc}
\hline Peak & Retention time & Area & Area\% \\
\hline 1 & 24.735 & 13451683 & 99.98 \\
2 & 28.587 & 3022 & 0.02 \\
\hline
\end{tabular}

$210 \mathrm{~nm}$ Detection 


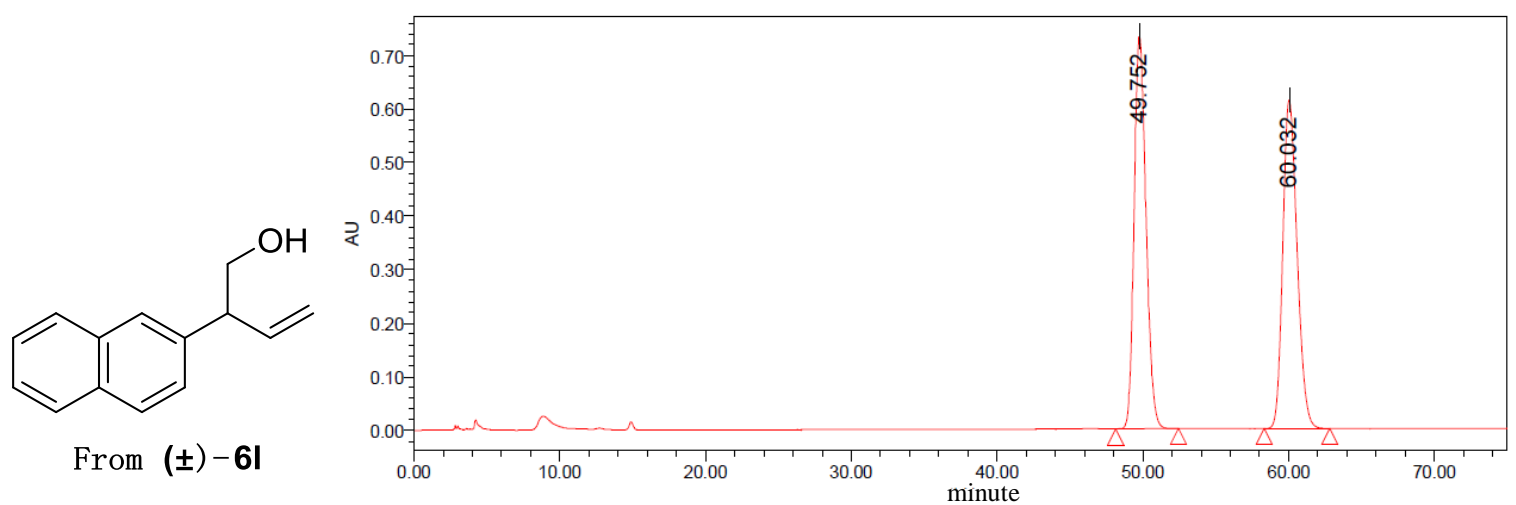

\begin{tabular}{cccc}
\hline Peak & Retention time & Area & Area\% \\
\hline 1 & 49.752 & 41486208 & 49.79 \\
2 & 60.032 & 41830621 & 50.21 \\
\hline
\end{tabular}

$210 \mathrm{~nm}$ Detection

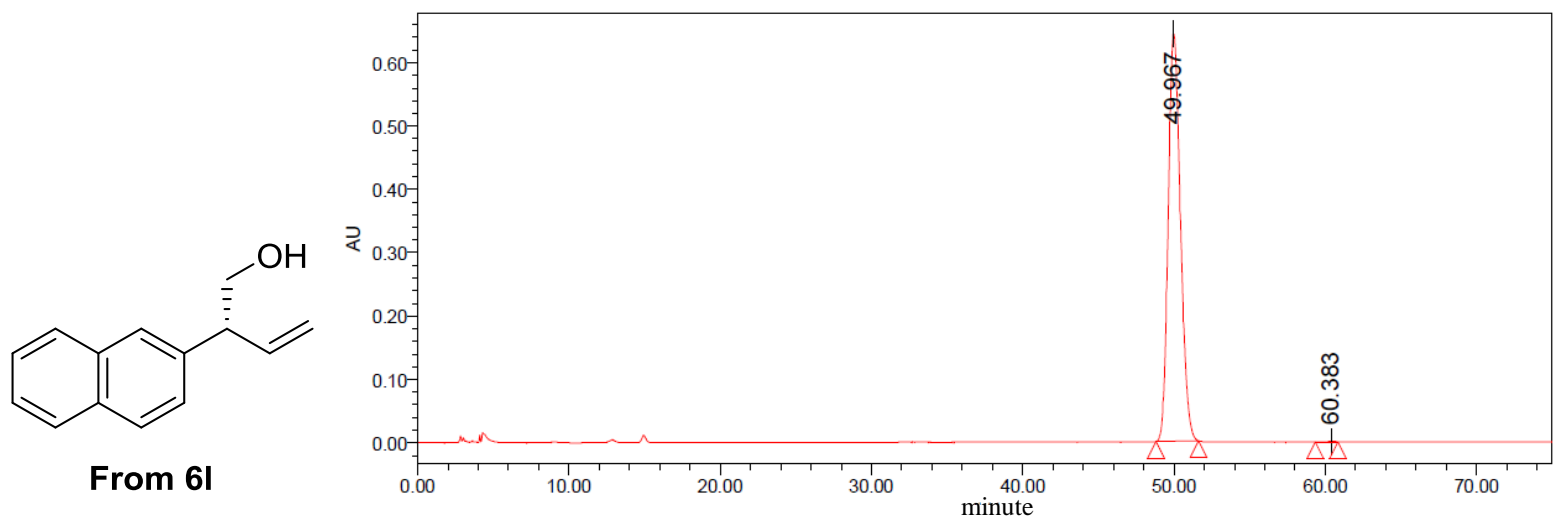

\begin{tabular}{cccc}
\hline Peak & Retention time & Area & Area\% \\
\hline 1 & 49.967 & 36170959 & 99.95 \\
2 & 60.383 & 17003 & 0.05 \\
\hline
\end{tabular}

$210 \mathrm{~nm}$ Detection 


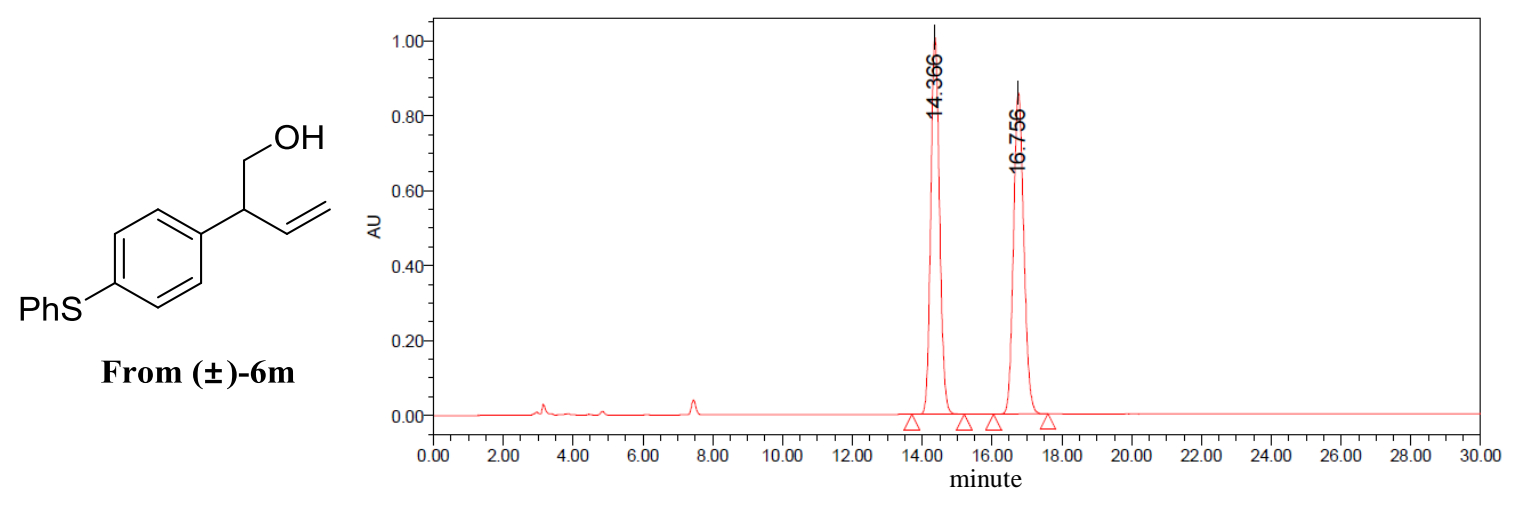

\begin{tabular}{cccc}
\hline Peak & Retention time & Area & Area\% \\
\hline 1 & 14.366 & 17625920 & 50.04 \\
2 & 16.756 & 17600612 & 49.96 \\
\hline
\end{tabular}

\section{$210 \mathrm{~nm}$ Detection}

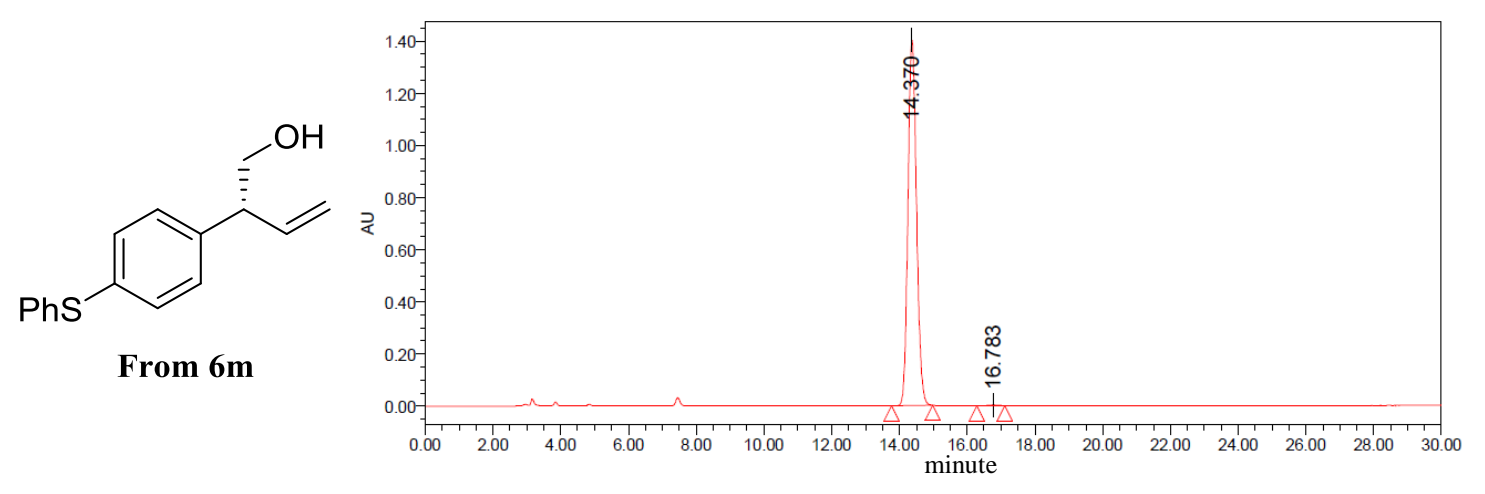

\begin{tabular}{cccc}
\hline Peak & Retention time & Area & Area\% \\
\hline 1 & 14.370 & 24594004 & 99.81 \\
2 & 16.783 & 46513 & 0.19 \\
\hline
\end{tabular}

$210 \mathrm{~nm}$ Detection 


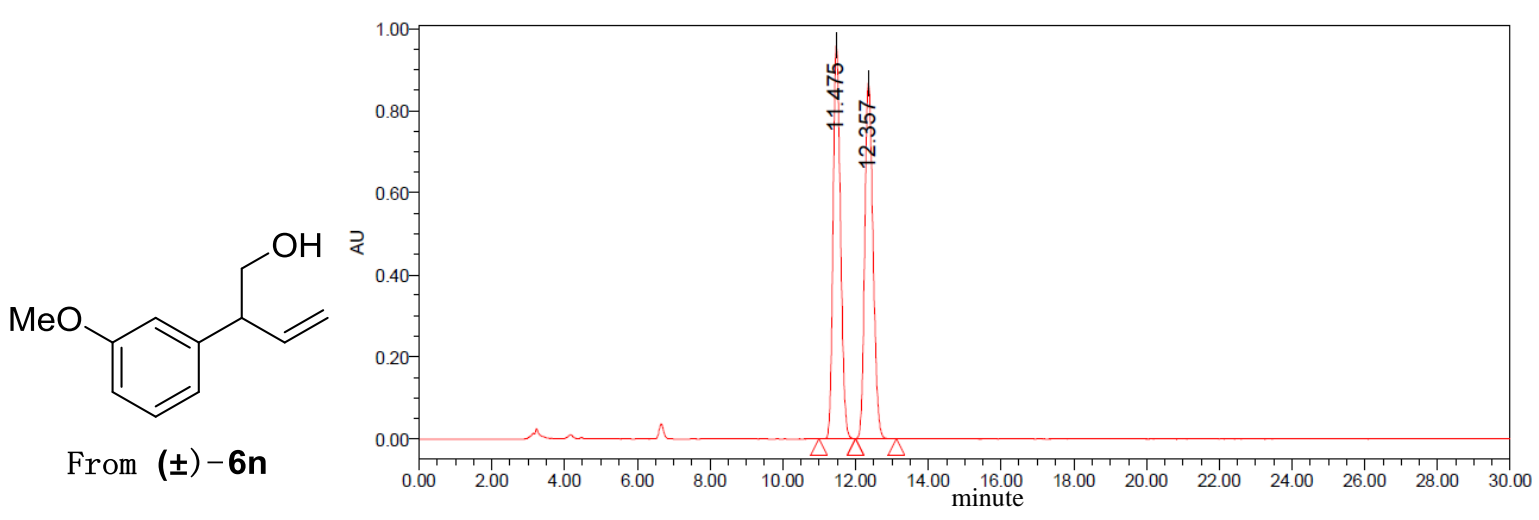

\begin{tabular}{cccc}
\hline Peak & Retention time & Area & Area\% \\
\hline 1 & 11.475 & 13677133 & 49.97 \\
2 & 12.357 & 13695136 & 50.03 \\
\hline
\end{tabular}

$210 \mathrm{~nm}$ Detection

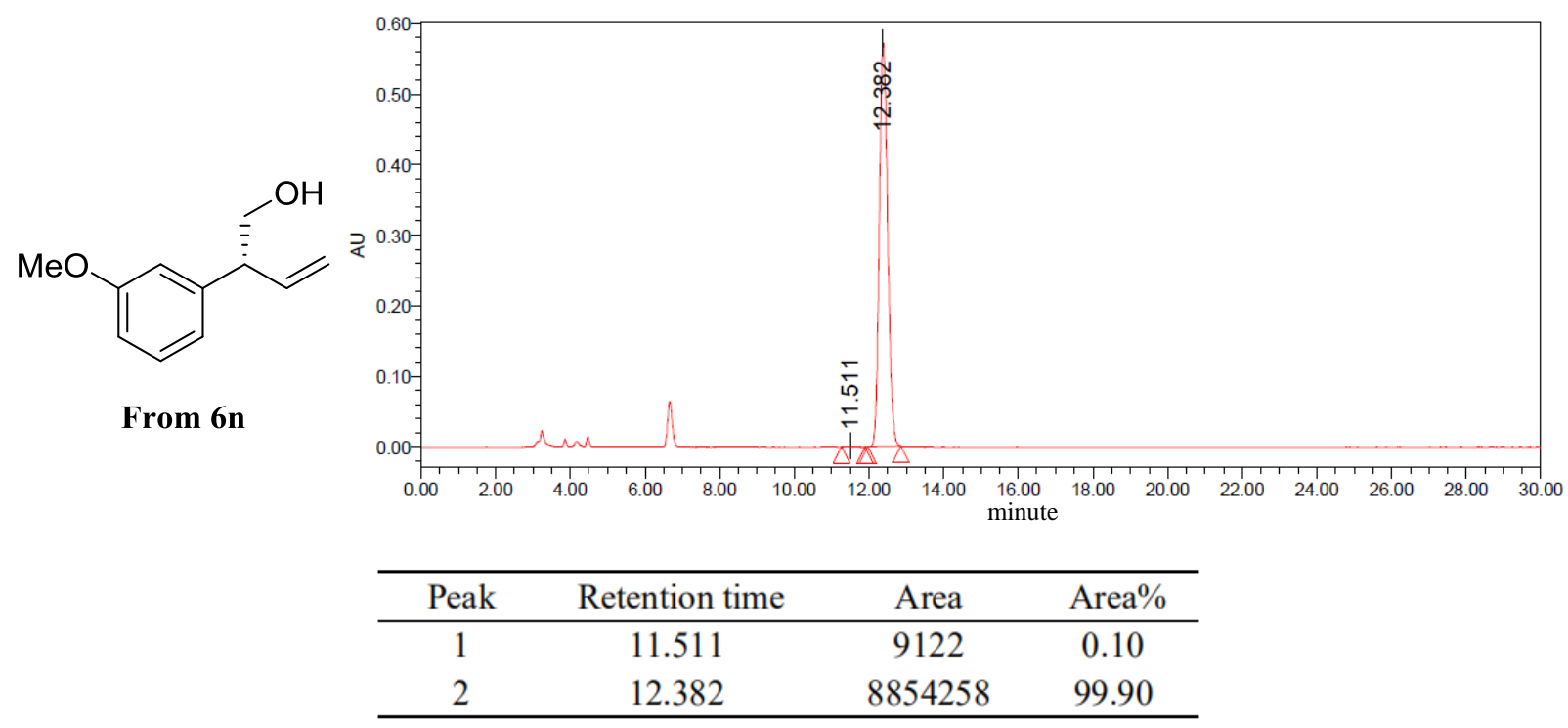

$210 \mathrm{~nm}$ Detection 


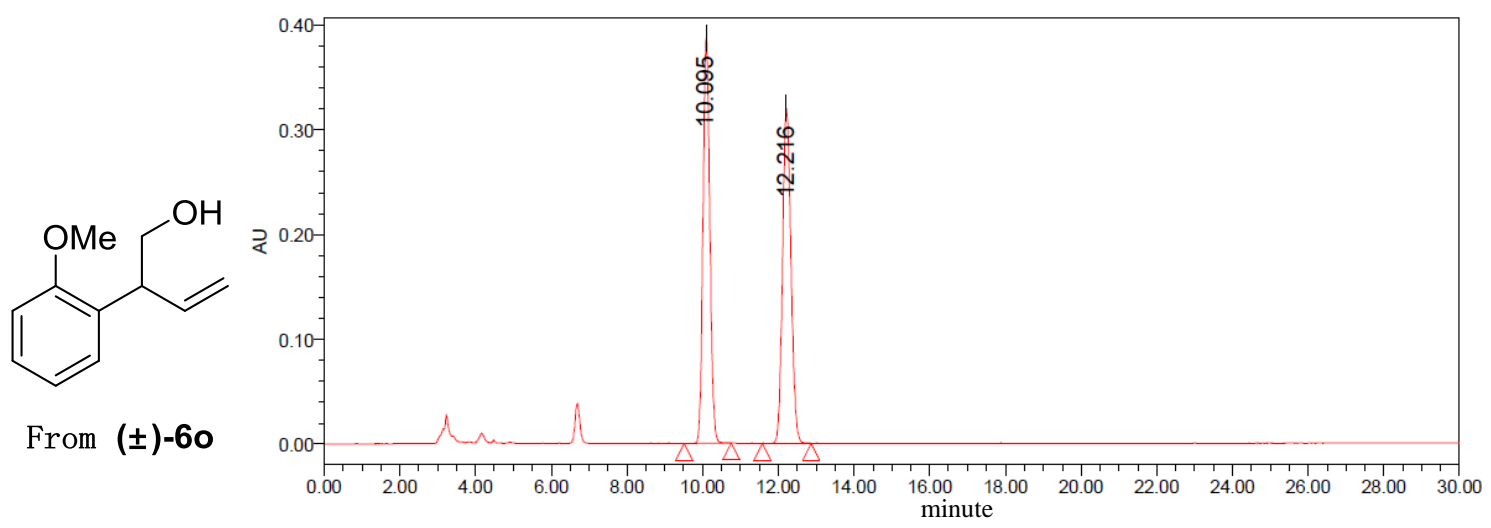

\begin{tabular}{cccc}
\hline Peak & Retention time & Area & Area\% \\
\hline 1 & 10.095 & 4838493 & 49.41 \\
2 & 12.216 & 4953209 & 50.59 \\
\hline
\end{tabular}

$210 \mathrm{~nm}$ Detection

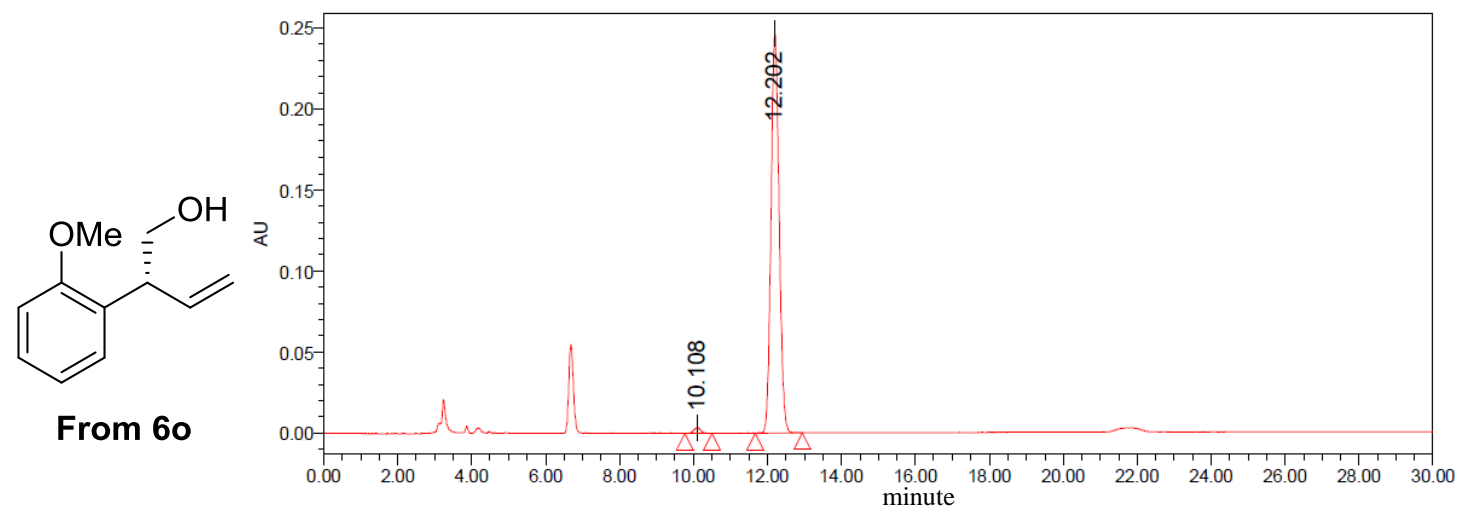

\begin{tabular}{cccc}
\hline Peak & Retention time & Area & Area\% \\
\hline 1 & 10.108 & 42247 & 1.11 \\
2 & 12.202 & 3767584 & 98.89 \\
\hline
\end{tabular}

$210 \mathrm{~nm}$ Detection 


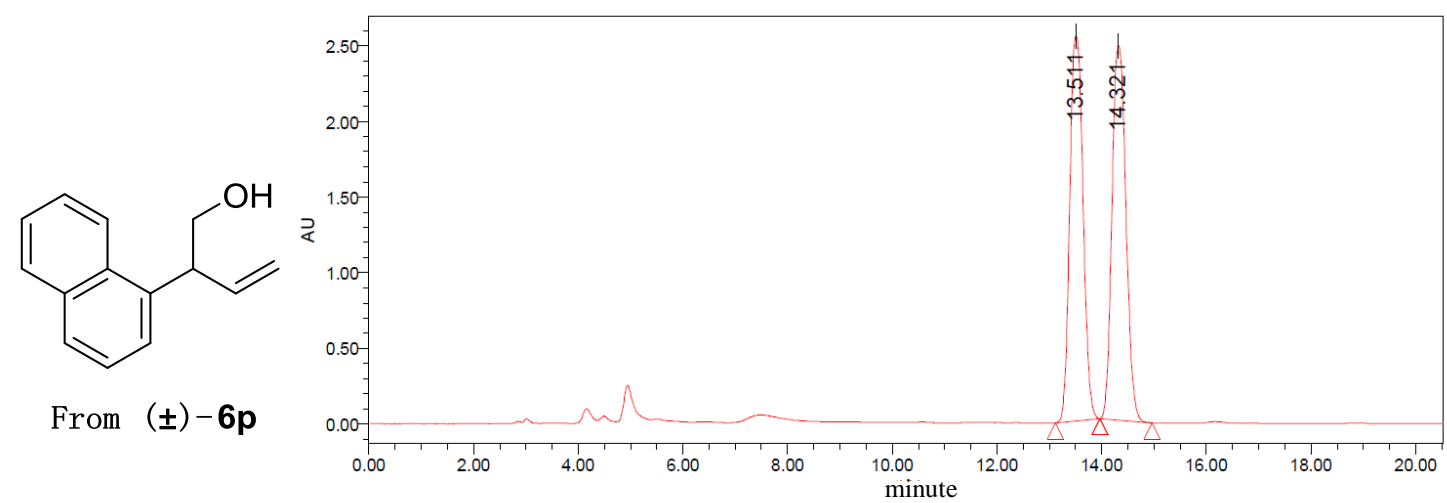

\begin{tabular}{cccc}
\hline Peak & Retention time & Area & Area\% \\
\hline 1 & 13.511 & 44946984 & 49.53 \\
2 & 14.321 & 45793568 & 50.47 \\
\hline
\end{tabular}

$210 \mathrm{~nm}$ Detection

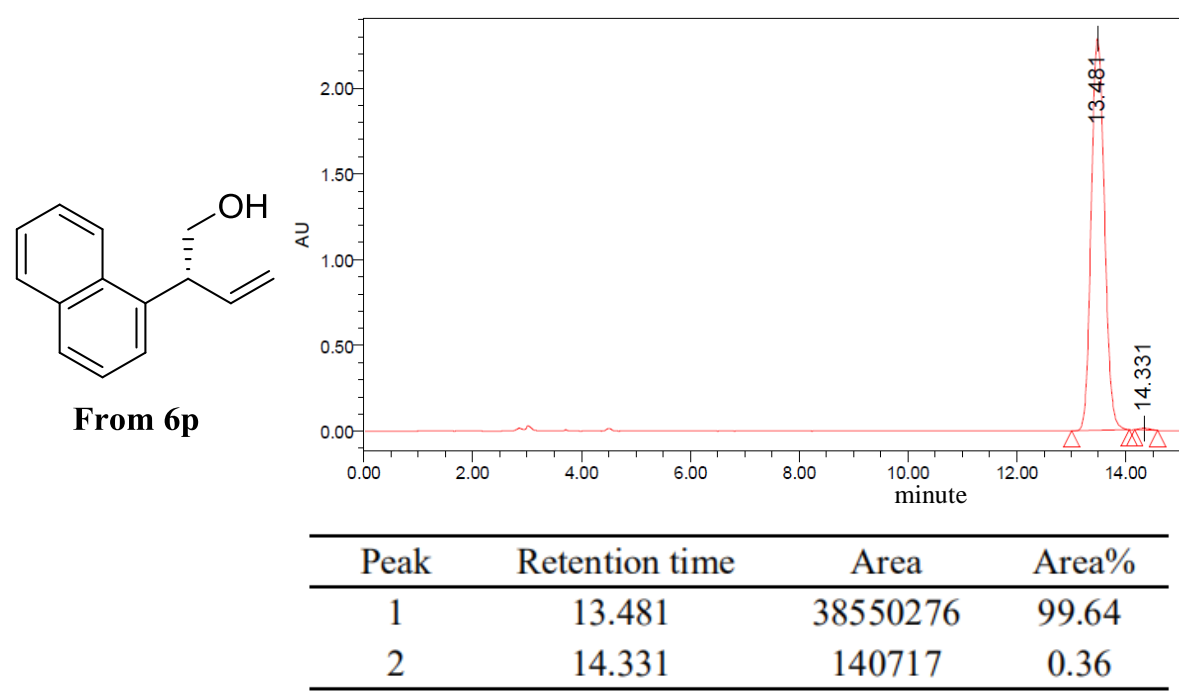

$210 \mathrm{~nm}$ Detection 


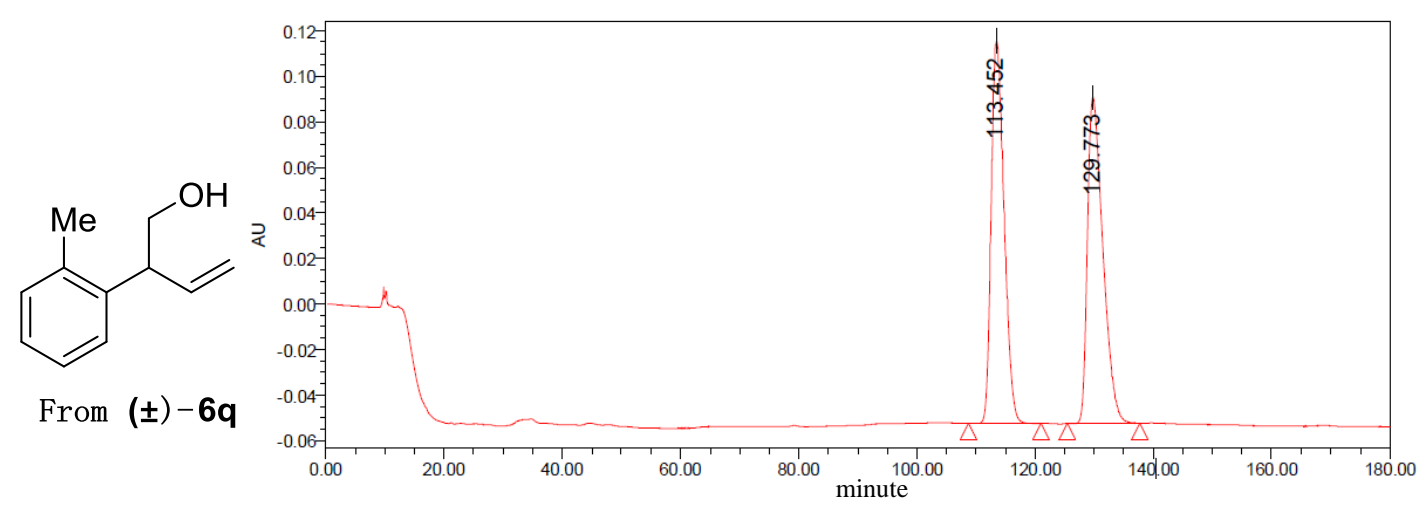

\begin{tabular}{cccc}
\hline Peak & Retention time & Area & Area $\%$ \\
\hline 1 & 113.452 & 25820545 & 50.03 \\
2 & 129.773 & 25786870 & 49.97 \\
\hline
\end{tabular}

\section{$210 \mathrm{~nm}$ Detection}

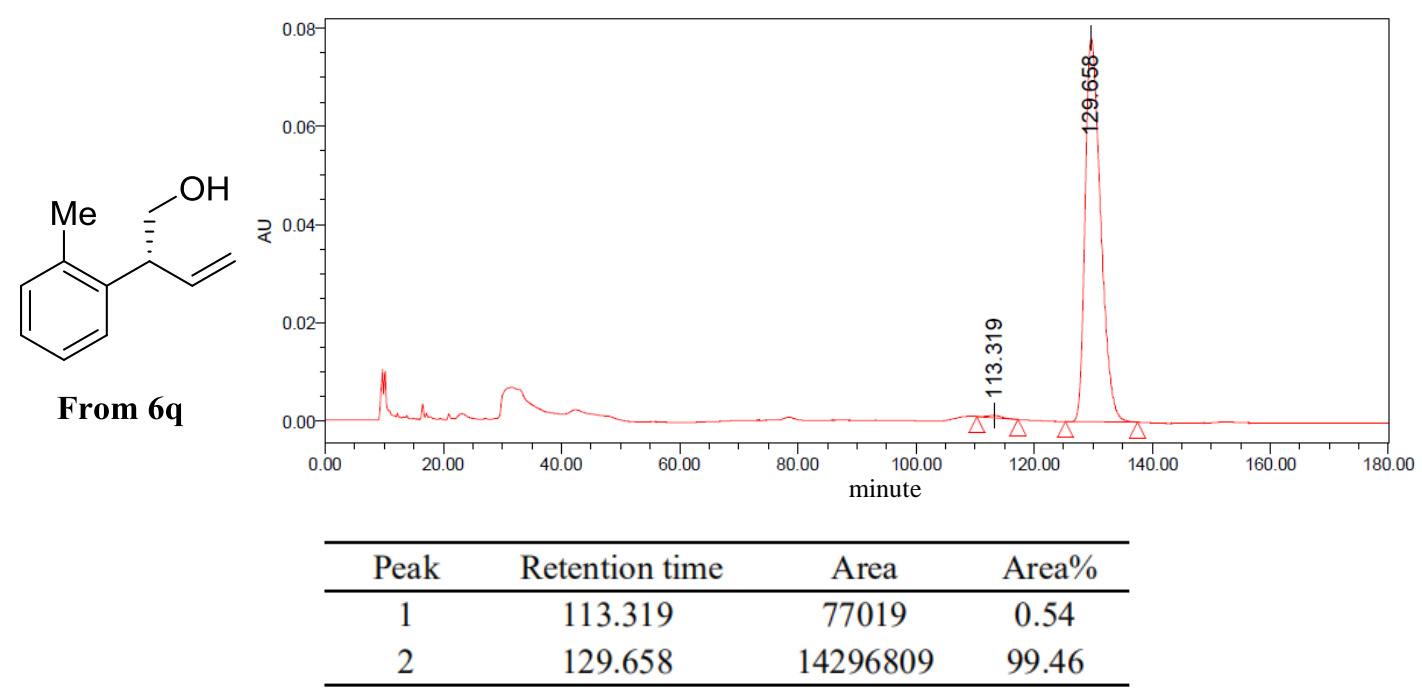

$210 \mathrm{~nm}$ Detection 


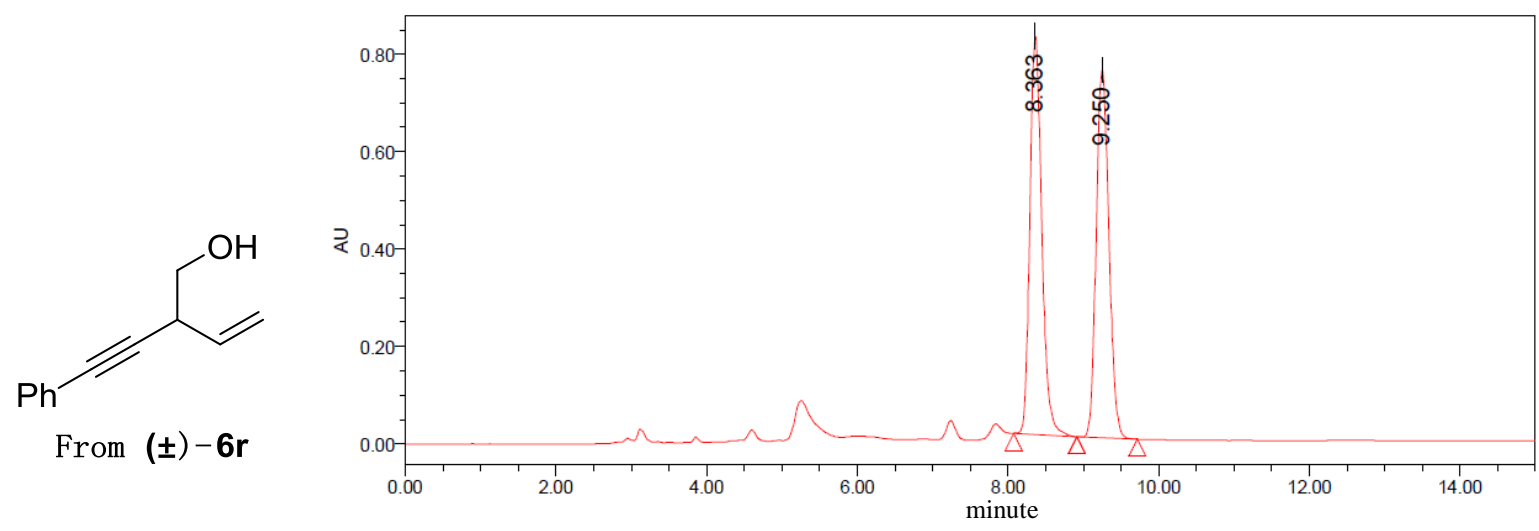

\begin{tabular}{cccc}
\hline Peak & Retention time & Area & Area\% \\
\hline 1 & 8.363 & 9000746 & 50.40 \\
2 & 9.250 & 8856376 & 49.60 \\
\hline
\end{tabular}

$210 \mathrm{~nm}$ Detection

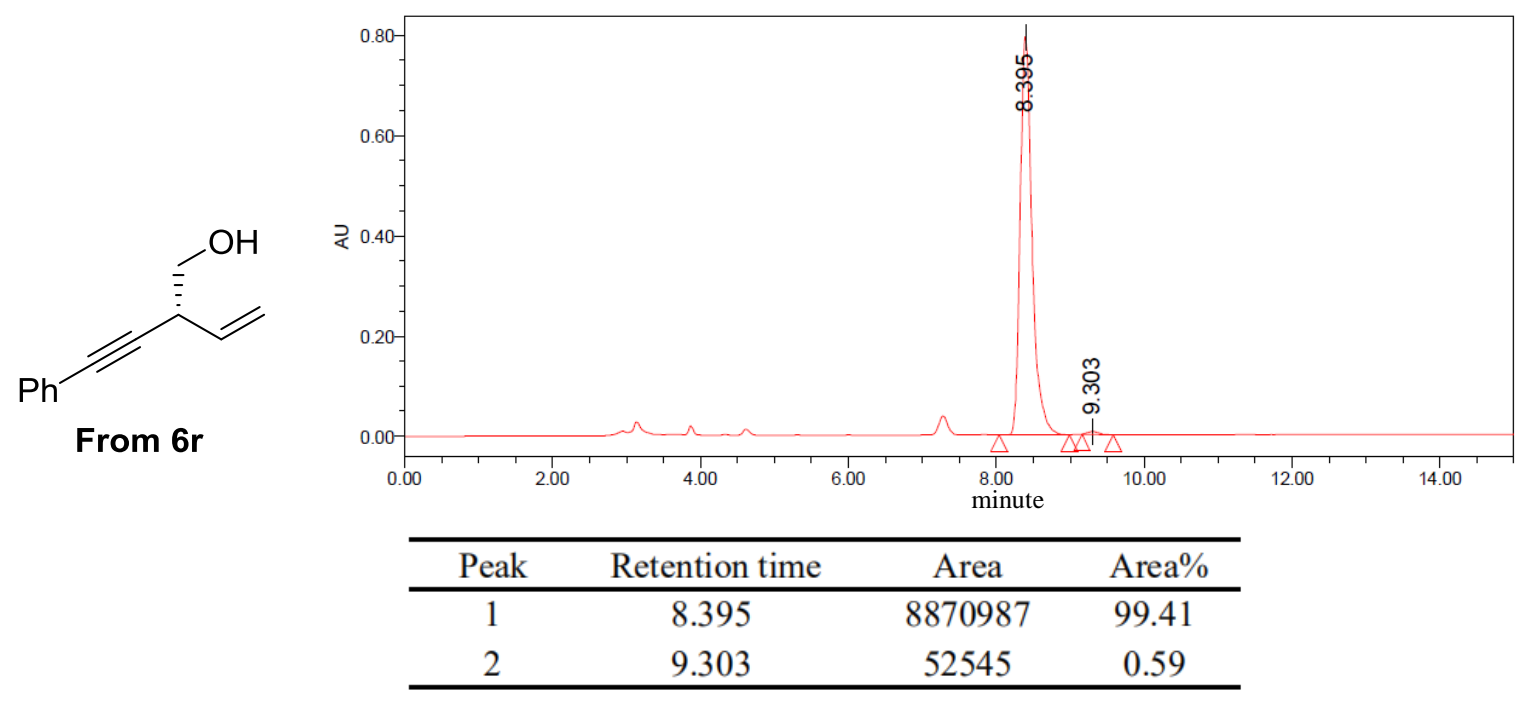

$210 \mathrm{~nm}$ Detection 


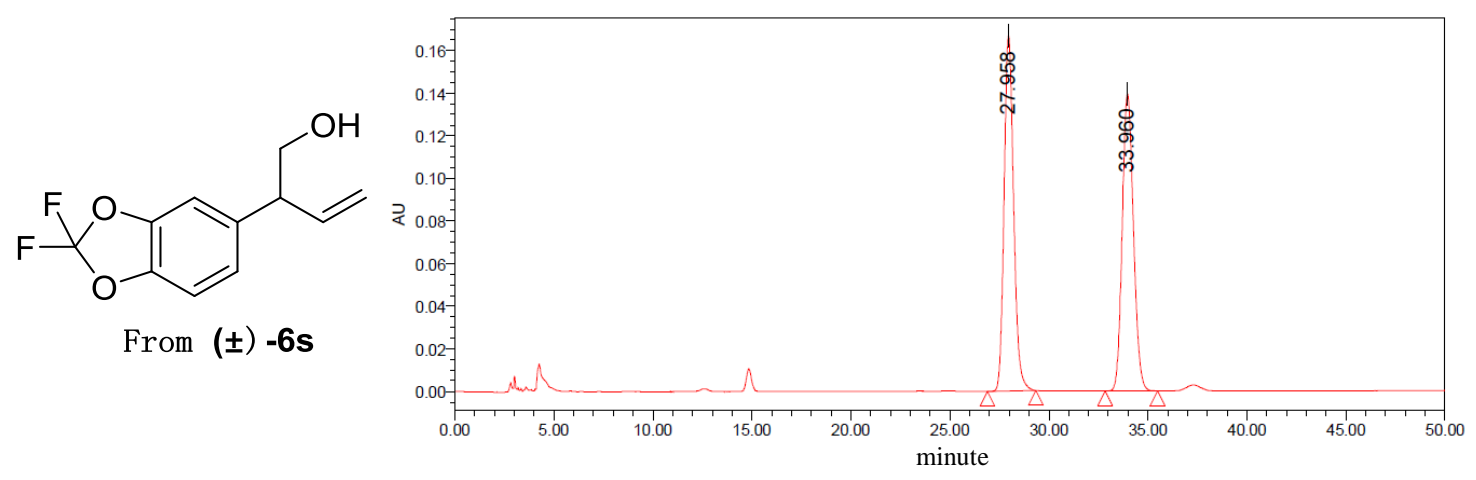

\begin{tabular}{cccc}
\hline Peak & Retention time & Area & Area $\%$ \\
\hline 1 & 27.958 & 5597530 & 50.58 \\
2 & 33.960 & 5470181 & 49.42 \\
\hline
\end{tabular}

\section{$210 \mathrm{~nm}$ Detection}

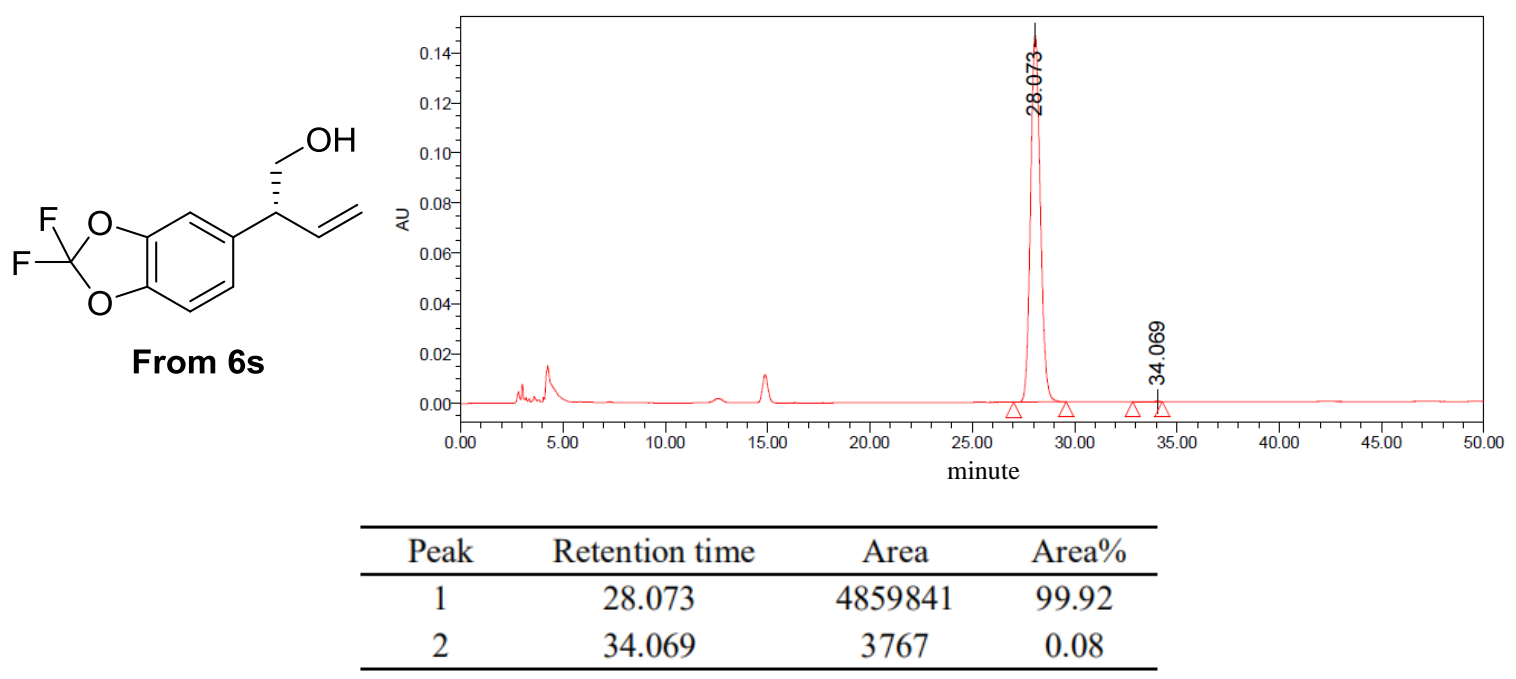

$210 \mathrm{~nm}$ Detection 


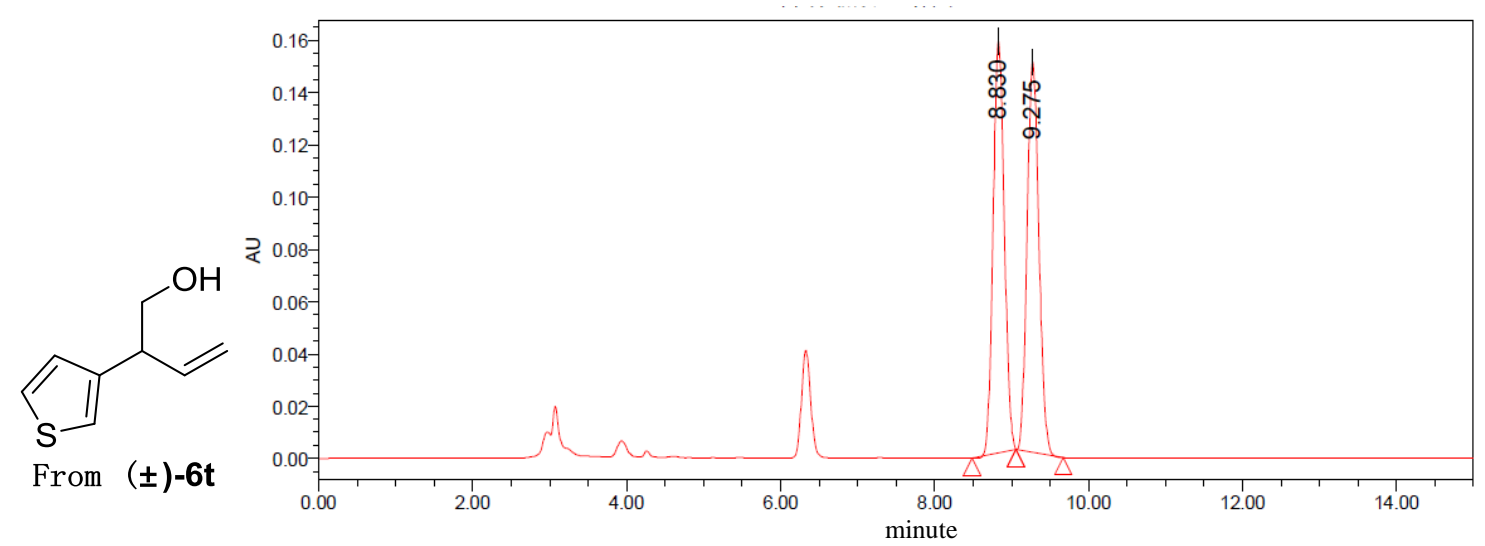

\begin{tabular}{cccc}
\hline Peak & Retention time & Area & Area\% \\
\hline 1 & 8.830 & 1590668 & 49.96 \\
2 & 9.275 & 1592919 & 50.04 \\
\hline
\end{tabular}

$210 \mathrm{~nm}$ Detection

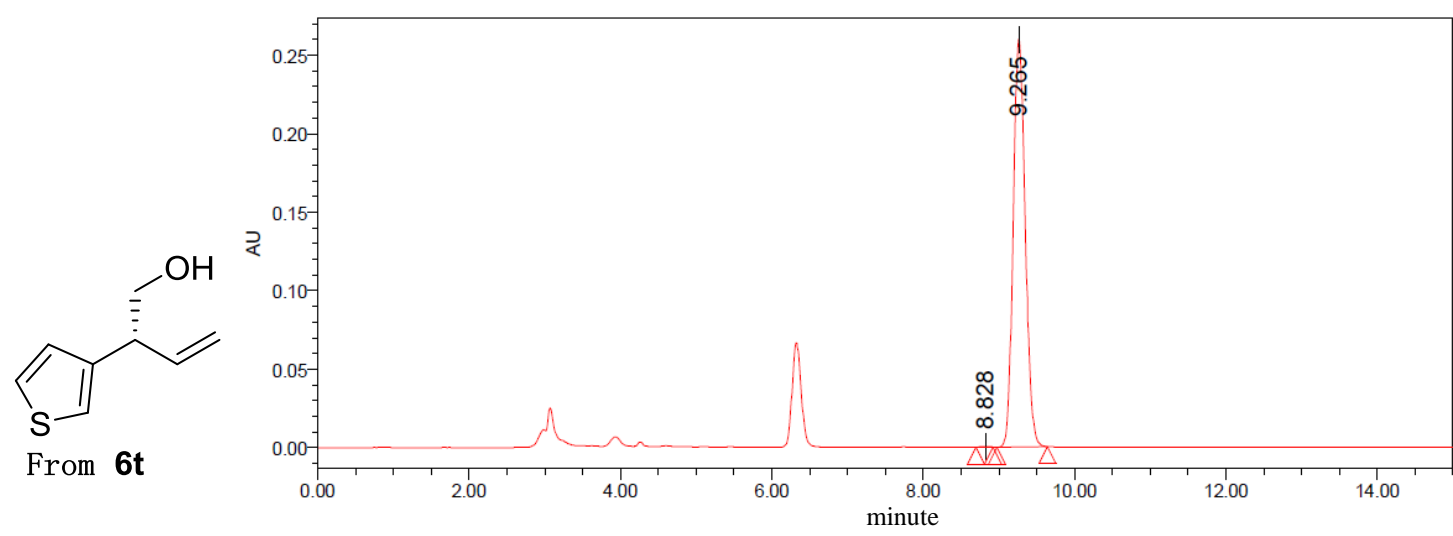

\begin{tabular}{cccc}
\hline Peak & Retention time & Area & Area\% \\
\hline 1 & 8.828 & 2117 & 0.07 \\
2 & 9.265 & 2863513 & 99.93 \\
\hline
\end{tabular}

$210 \mathrm{~nm}$ Detection 
<smiles>C=CC(CO)c1cccnc1</smiles>

From ( \pm ) -6u

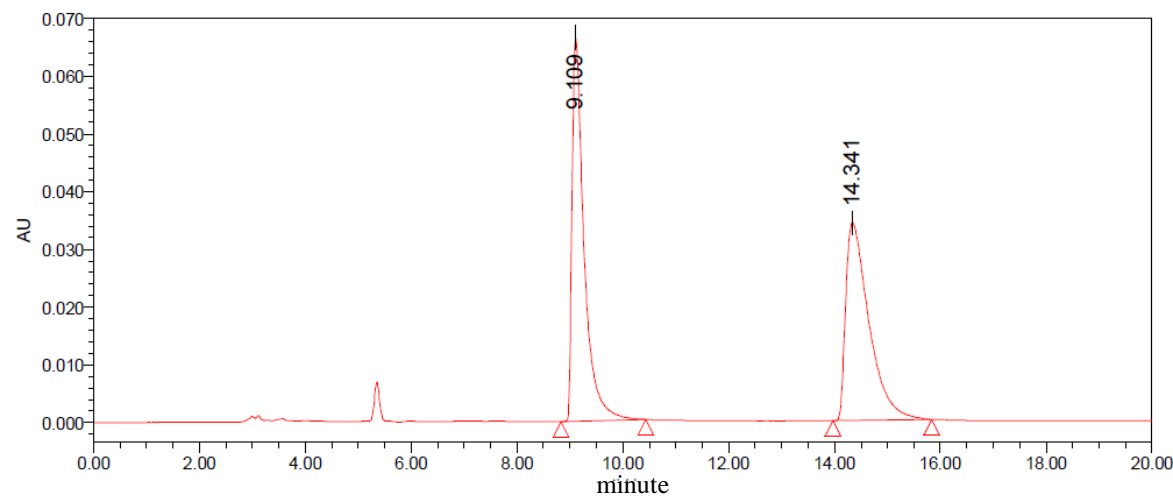

\begin{tabular}{cccc}
\hline Peak & Retention time & Area & Area\% \\
\hline 1 & 9.109 & 1048618 & 50.00 \\
2 & 14.341 & 1048634 & 50.00 \\
\hline
\end{tabular}

$254 \mathrm{~nm}$ Detection

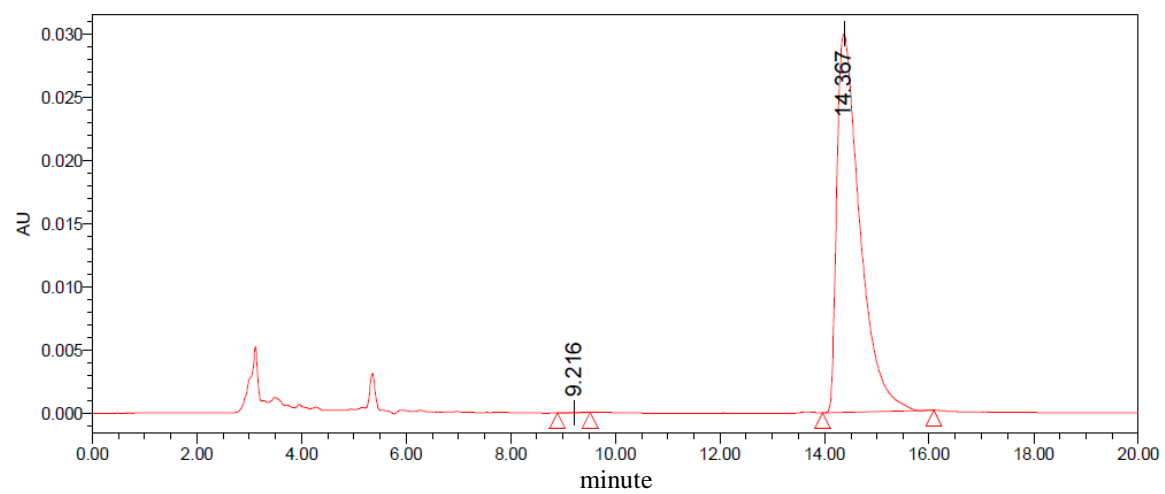

\begin{tabular}{cccc}
\hline Peak & Retention time & Area & Area\% \\
\hline 1 & 9.216 & 181 & 0.02 \\
2 & 14.367 & 917067 & 99.98 \\
\hline
\end{tabular}

$254 \mathrm{~nm}$ Detection 


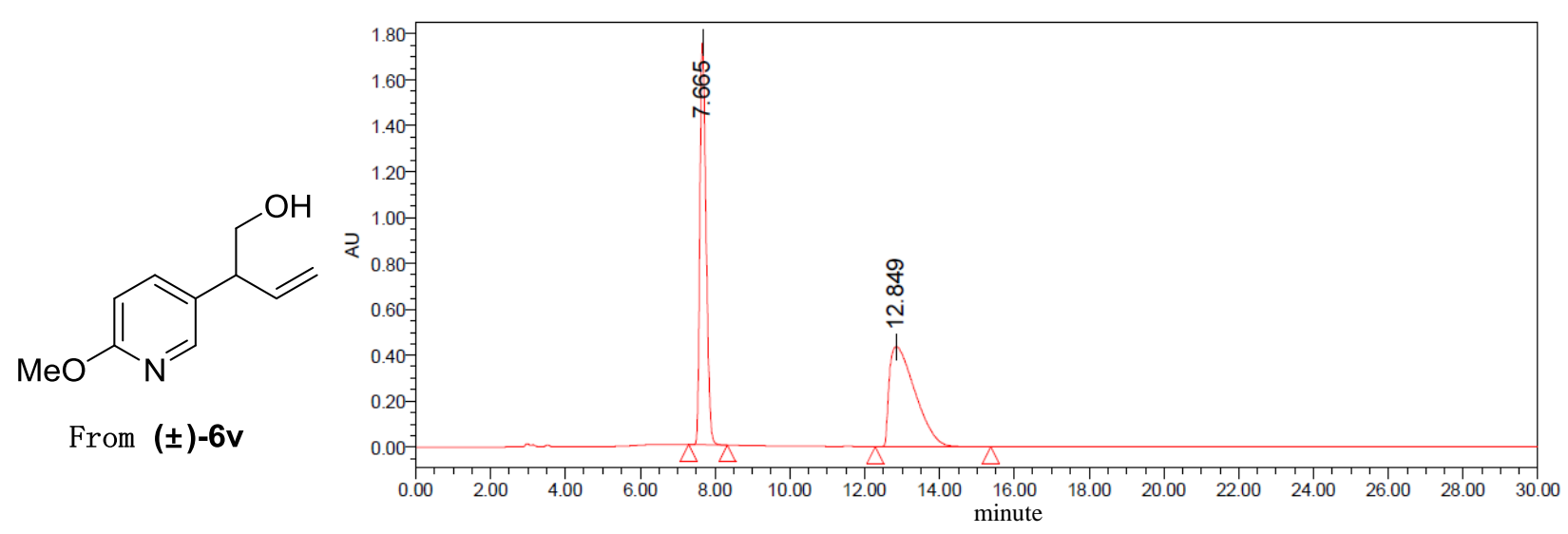

\begin{tabular}{cccc}
\hline Peak & Retention time & Area & Area\% \\
\hline 1 & 7.665 & 20133063 & 49.32 \\
2 & 12.849 & 20685559 & 50.68 \\
\hline
\end{tabular}

$220 \mathrm{~nm}$ Detection

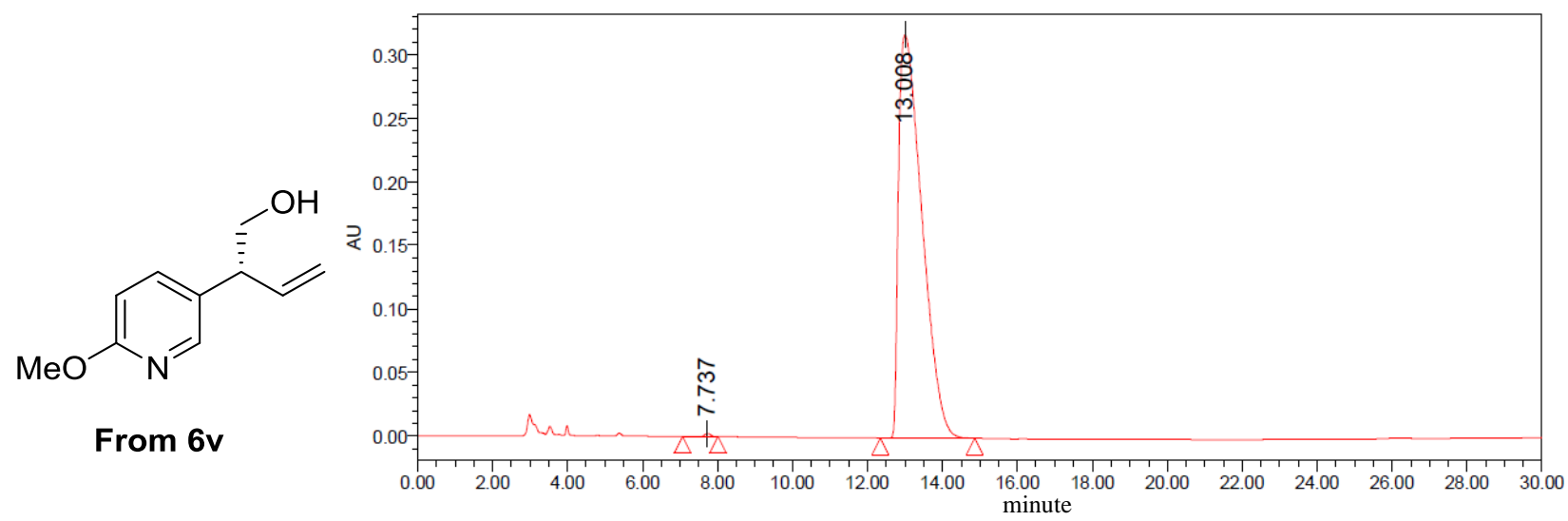

\begin{tabular}{cccc}
\hline Peak & Retention time & Area & Area\% \\
\hline 1 & 7.737 & 27089 & 0.20 \\
2 & 13.008 & 13685397 & 99.80 \\
\hline
\end{tabular}

$220 \mathrm{~nm}$ Detection 


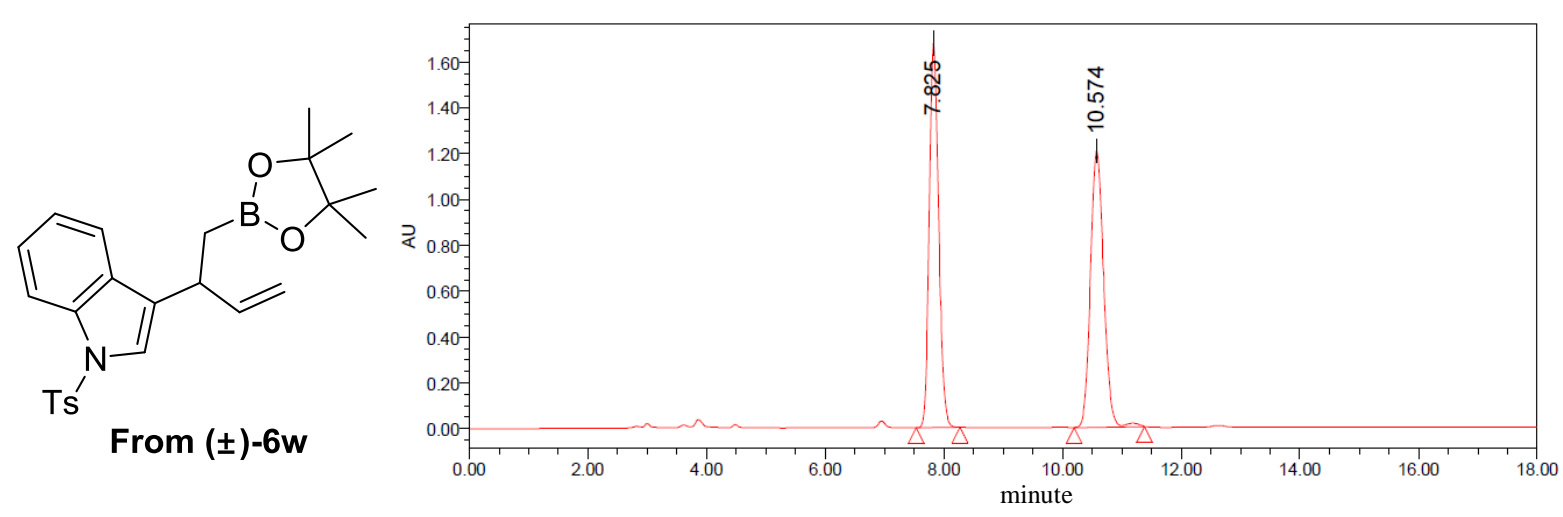

\begin{tabular}{cccc}
\hline Peak & Retention time & Area & Area\% \\
\hline 1 & 7.825 & 18491449 & 49.71 \\
2 & 10.574 & 18705467 & 50.29 \\
\hline
\end{tabular}

$210 \mathrm{~nm}$ Detection

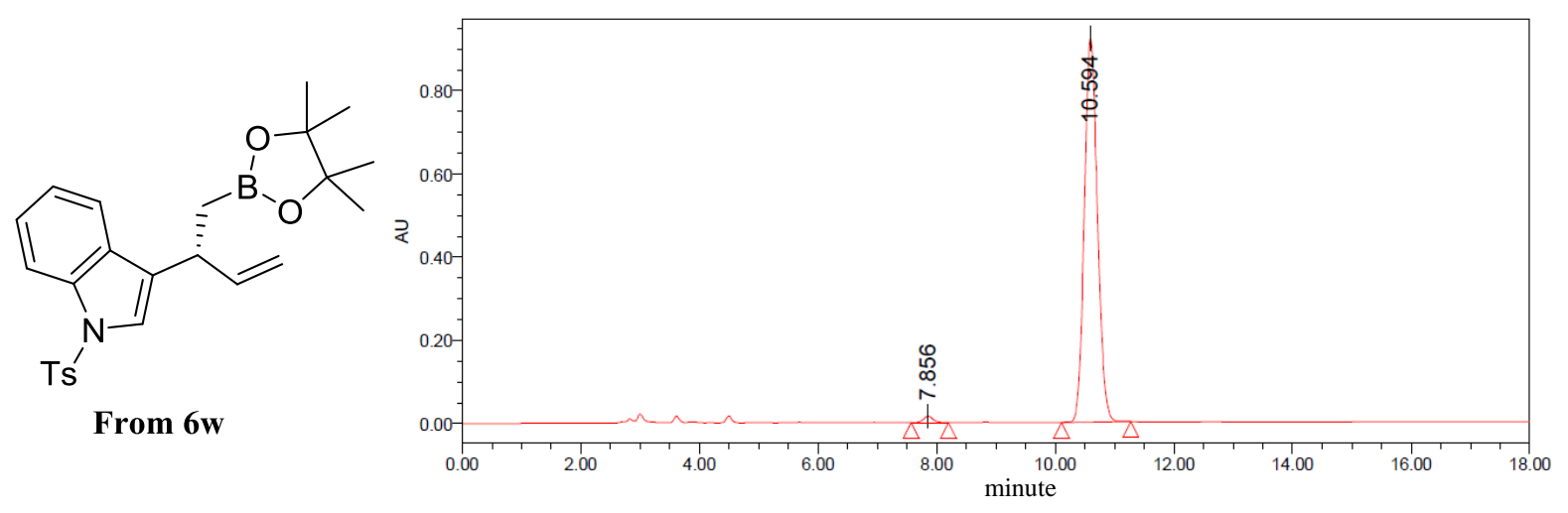

\begin{tabular}{cccc}
\hline Peak & Retention time & Area & Area\% \\
\hline 1 & 7.856 & 167989 & 1.17 \\
2 & 10.594 & 14129752 & 98.83 \\
\hline
\end{tabular}

$210 \mathrm{~nm}$ Detection 


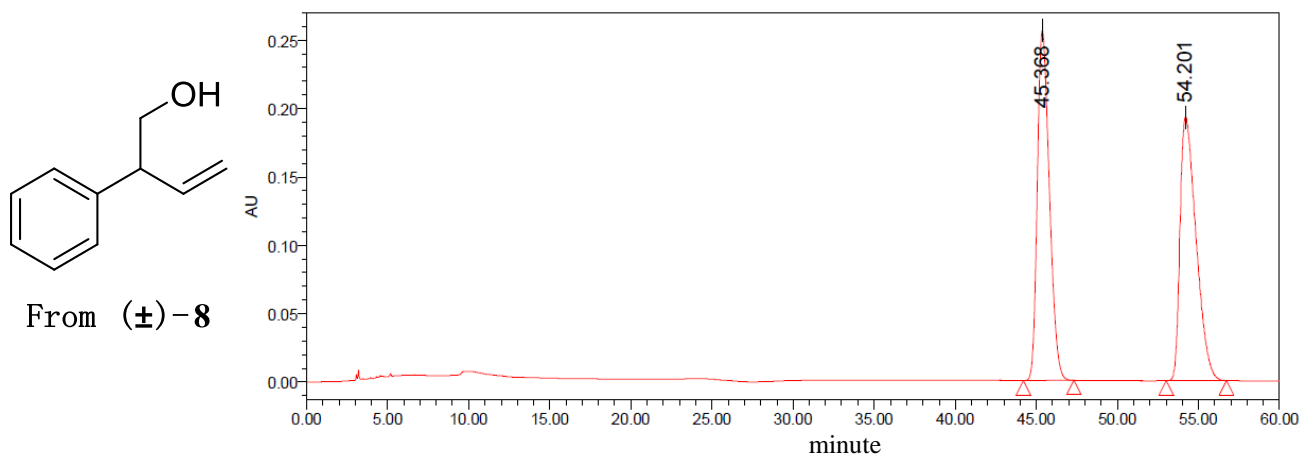

\begin{tabular}{cccc}
\hline Peak & Retention time & Area & Area\% \\
\hline 1 & 45.368 & 13247925 & 49.98 \\
2 & 54.201 & 13257139 & 50.02 \\
\hline
\end{tabular}

210 nm Detection

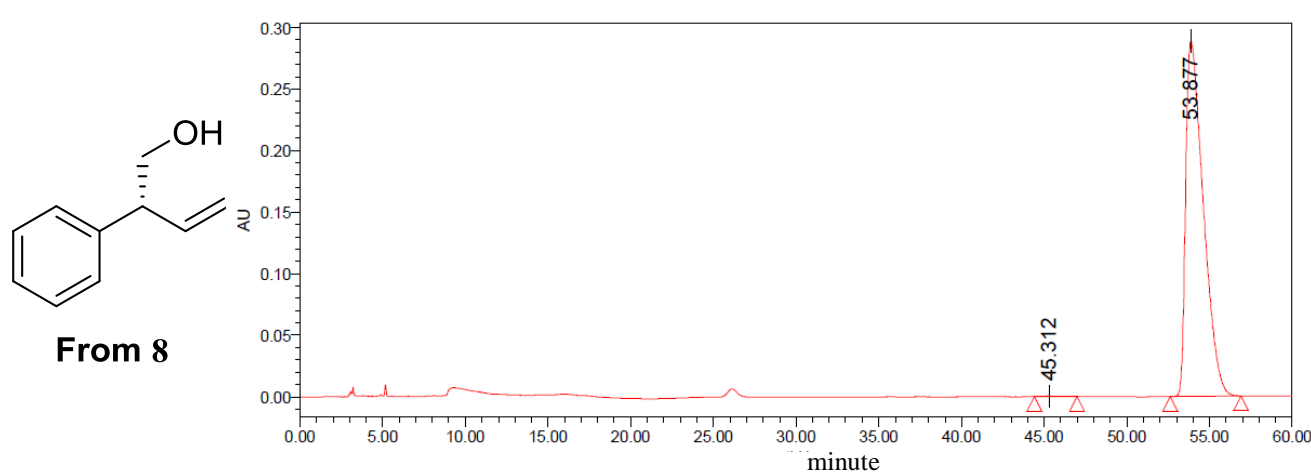

\begin{tabular}{cccc}
\hline Peak & Retention time & Area & Area\% \\
\hline 1 & 45.312 & 15437 & 0.07 \\
2 & 53.877 & 21275445 & 99.93 \\
\hline
\end{tabular}

$210 \mathrm{~nm}$ Detection 


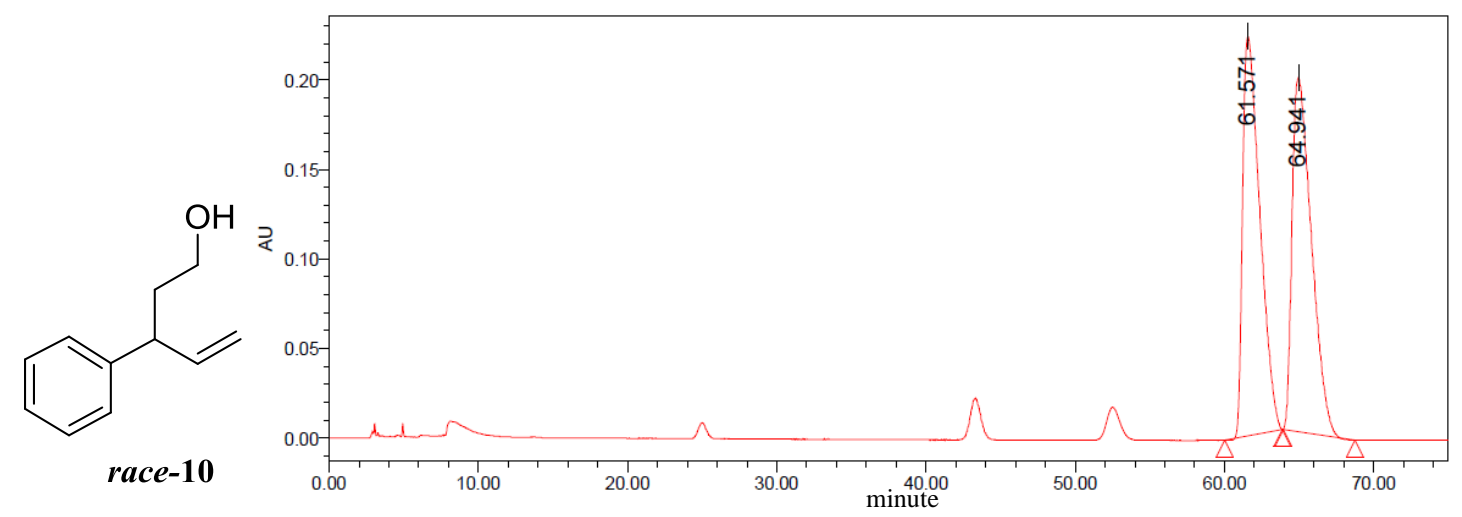

\begin{tabular}{cccc}
\hline Peak & Retention time & Area & Area\% \\
\hline 1 & 61.571 & 17928641 & 50.11 \\
2 & 64.941 & 17848793 & 49.89 \\
\hline
\end{tabular}

$210 \mathrm{~nm}$ Detection

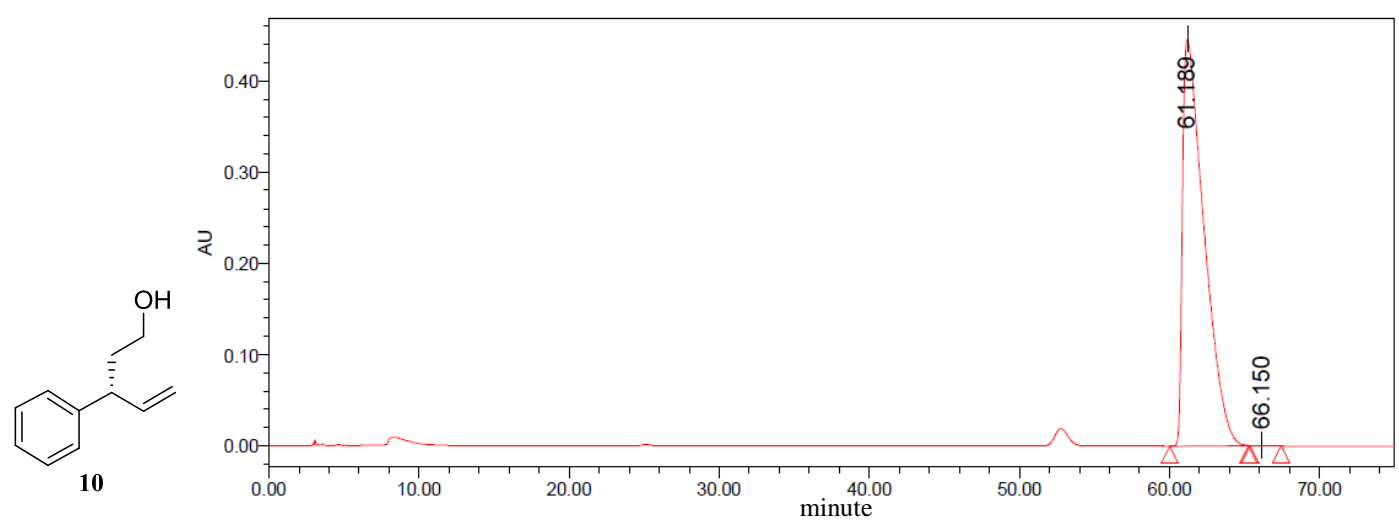

\begin{tabular}{cccc}
\hline Peak & Retention time & Area & Area\% \\
\hline 1 & 61.189 & 44420489 & 99.99 \\
2 & 66.150 & 3186 & 0.01 \\
\hline
\end{tabular}

$210 \mathrm{~nm}$ Detection 


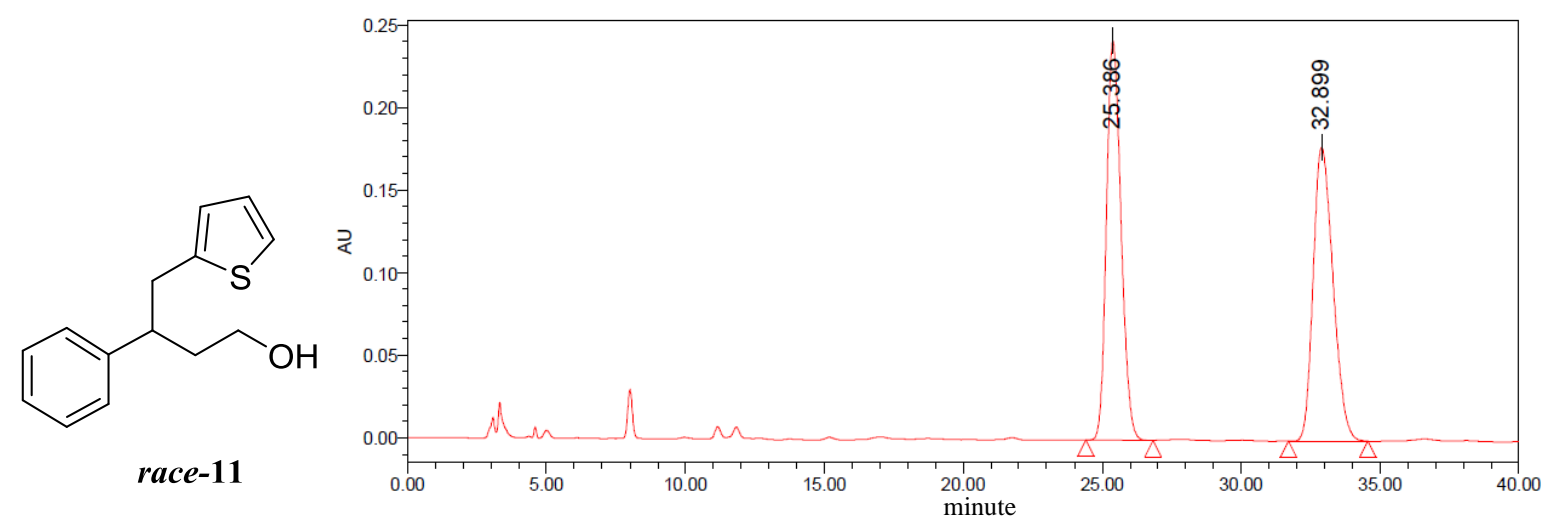

\begin{tabular}{cccc}
\hline Peak & Retention time & Area & Area\% \\
\hline 1 & 25.386 & 8811121 & 49.90 \\
2 & 32.899 & 8847095 & 50.10 \\
\hline
\end{tabular}

$210 \mathrm{~nm}$ Detection
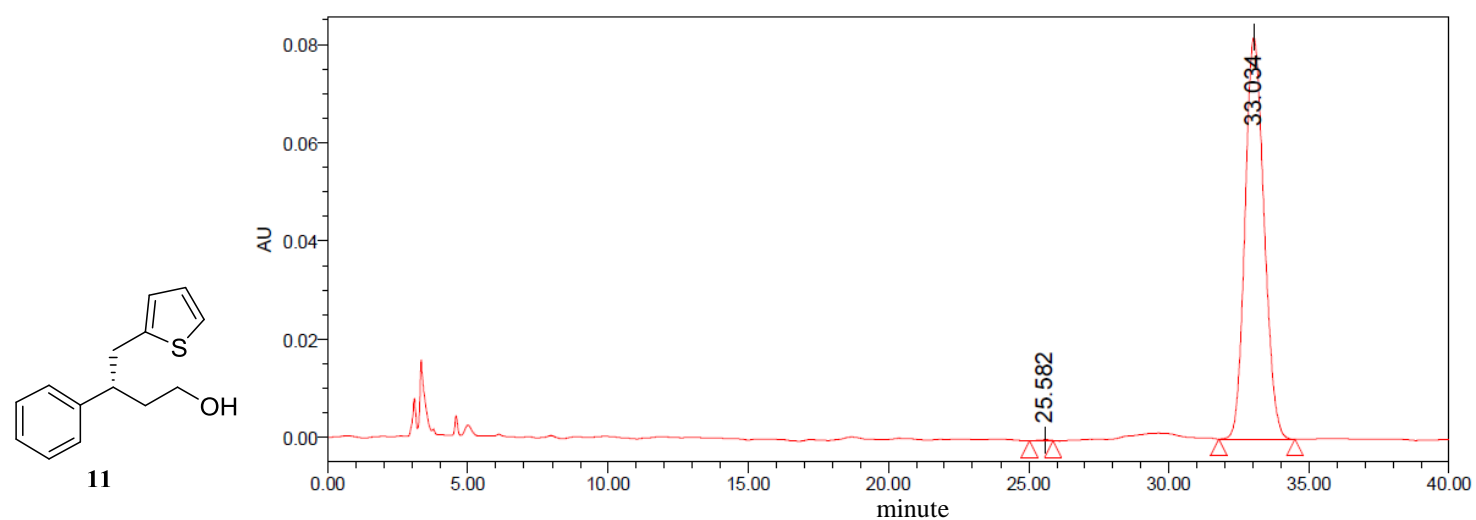

\begin{tabular}{cccc}
\hline Peak & Retention time & Area & Area $\%$ \\
\hline 1 & 25.582 & 2838 & 0.07 \\
2 & 33.034 & 3862965 & 99.93 \\
\hline
\end{tabular}

$210 \mathrm{~nm}$ Detection 


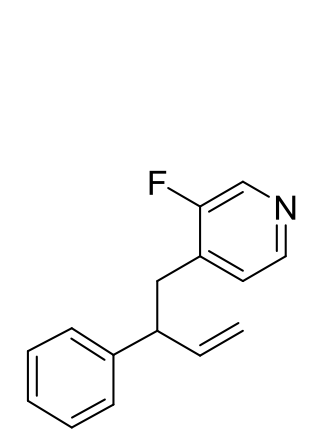

race-12

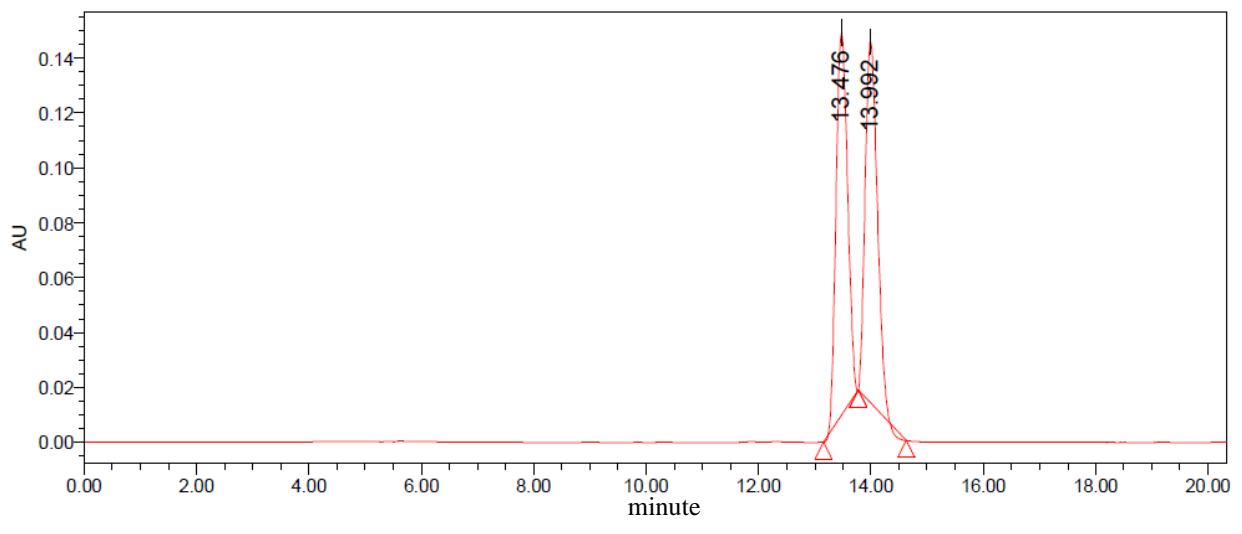

\begin{tabular}{cccc}
\hline Peak & Retention time & Area & Area\% \\
\hline 1 & 13.476 & 1983804 & 49.63 \\
2 & 13.992 & 2013171 & 50.37 \\
\hline
\end{tabular}

$254 \mathrm{~nm}$ Detection

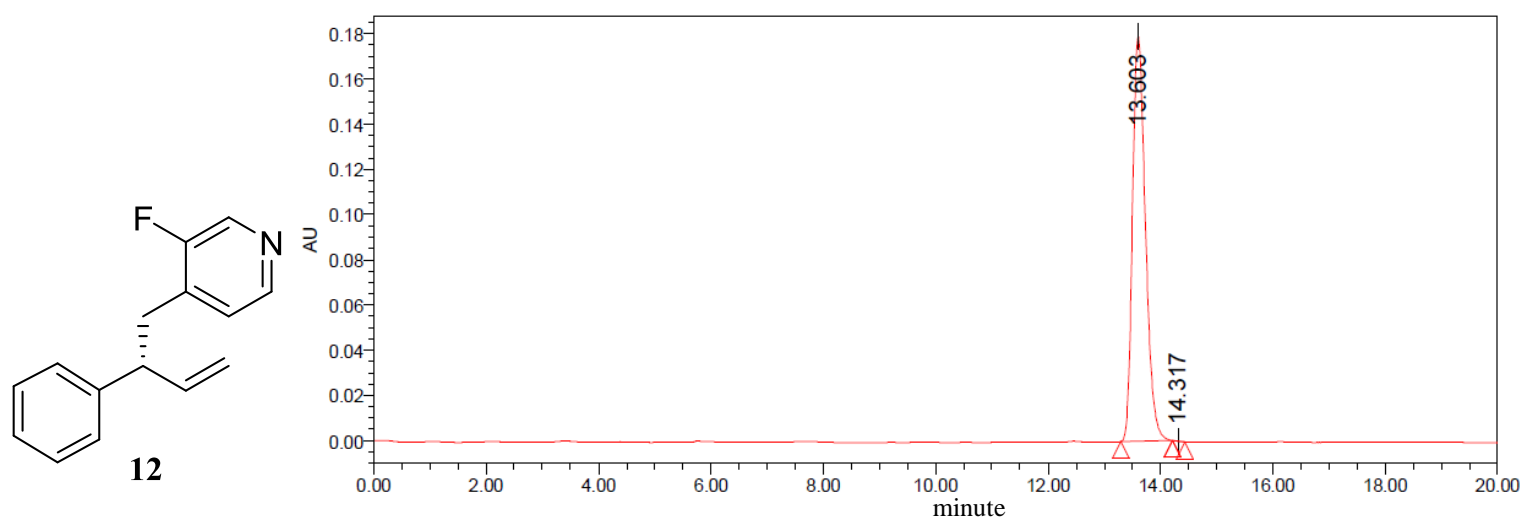

\begin{tabular}{cccc}
\hline Peak & Retention time & Area & Area\% \\
\hline 1 & 13.603 & 2865317 & 99.97 \\
2 & 14.317 & 773 & 0.03 \\
\hline
\end{tabular}

$254 \mathrm{~nm}$ Detection 
in $\mathrm{CDCl}_{3}, 400 \mathrm{MHz}$

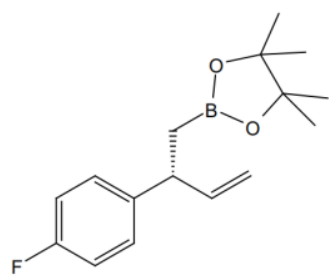

$6 a$

I I<smiles>[CH]1CC1</smiles>

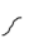

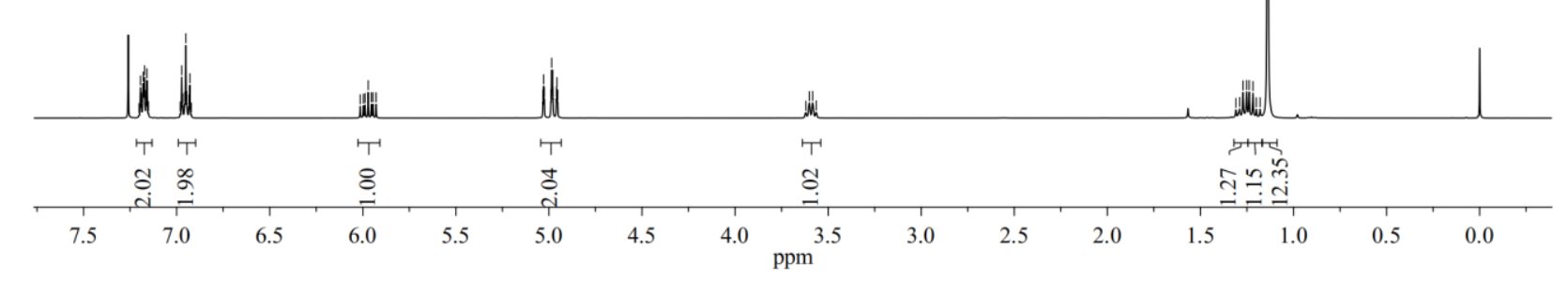

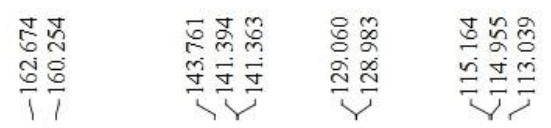

in $\mathrm{CDCl}_{3}, 101 \mathrm{MHz}$

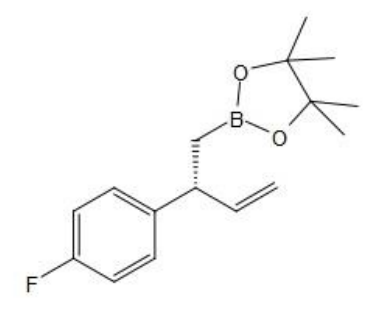

$6 a$

\begin{tabular}{|c|c|c|c|c|c|c|c|c|c|c|c|c|c|c|c|c|}
\hline 170 & 160 & 150 & 140 & 130 & 120 & 110 & 100 & 90 & 80 & 70 & $\begin{array}{l}1 \\
60\end{array}$ & 50 & 40 & 30 & 20 & 10 \\
\hline
\end{tabular}


in $\mathrm{CDCl}_{3}, 376 \mathrm{MHz}$

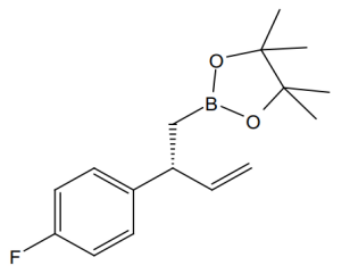

$6 a$
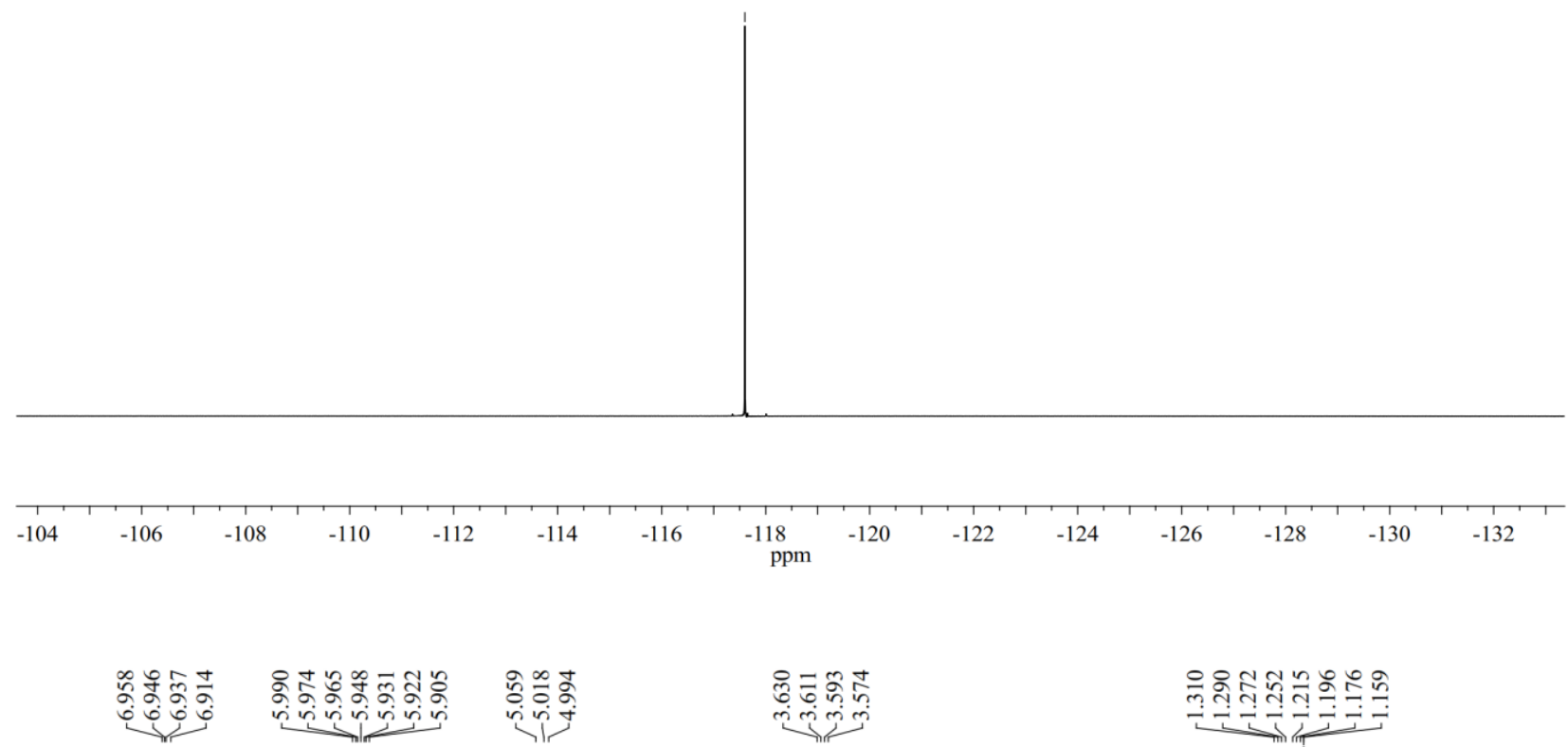

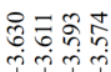

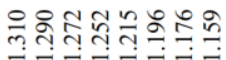

in $\mathrm{CDCl}_{3}, 400 \mathrm{MHz}$

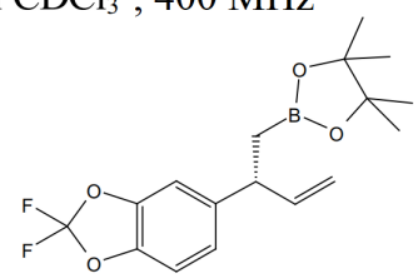

$6 s$<smiles>[CH]CC</smiles>

s
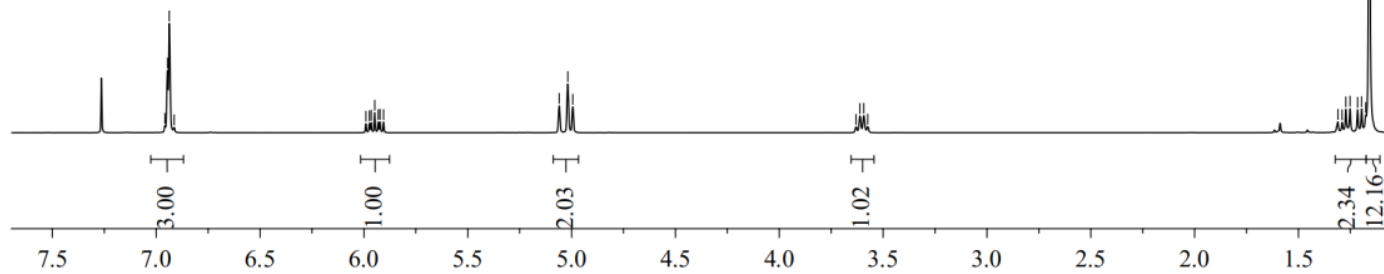

0 

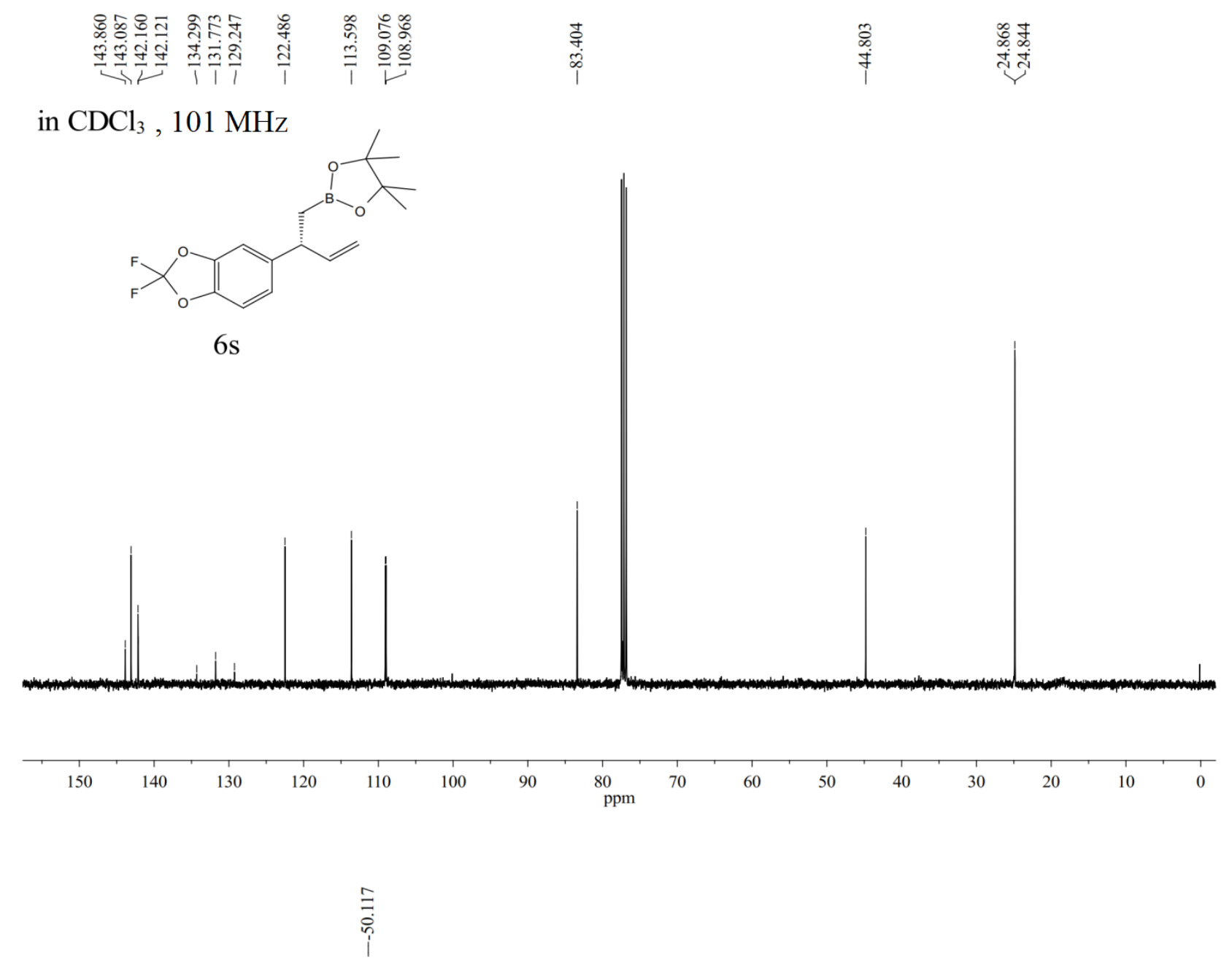

in $\mathrm{CDCl}_{3}, 376 \mathrm{MHz}$

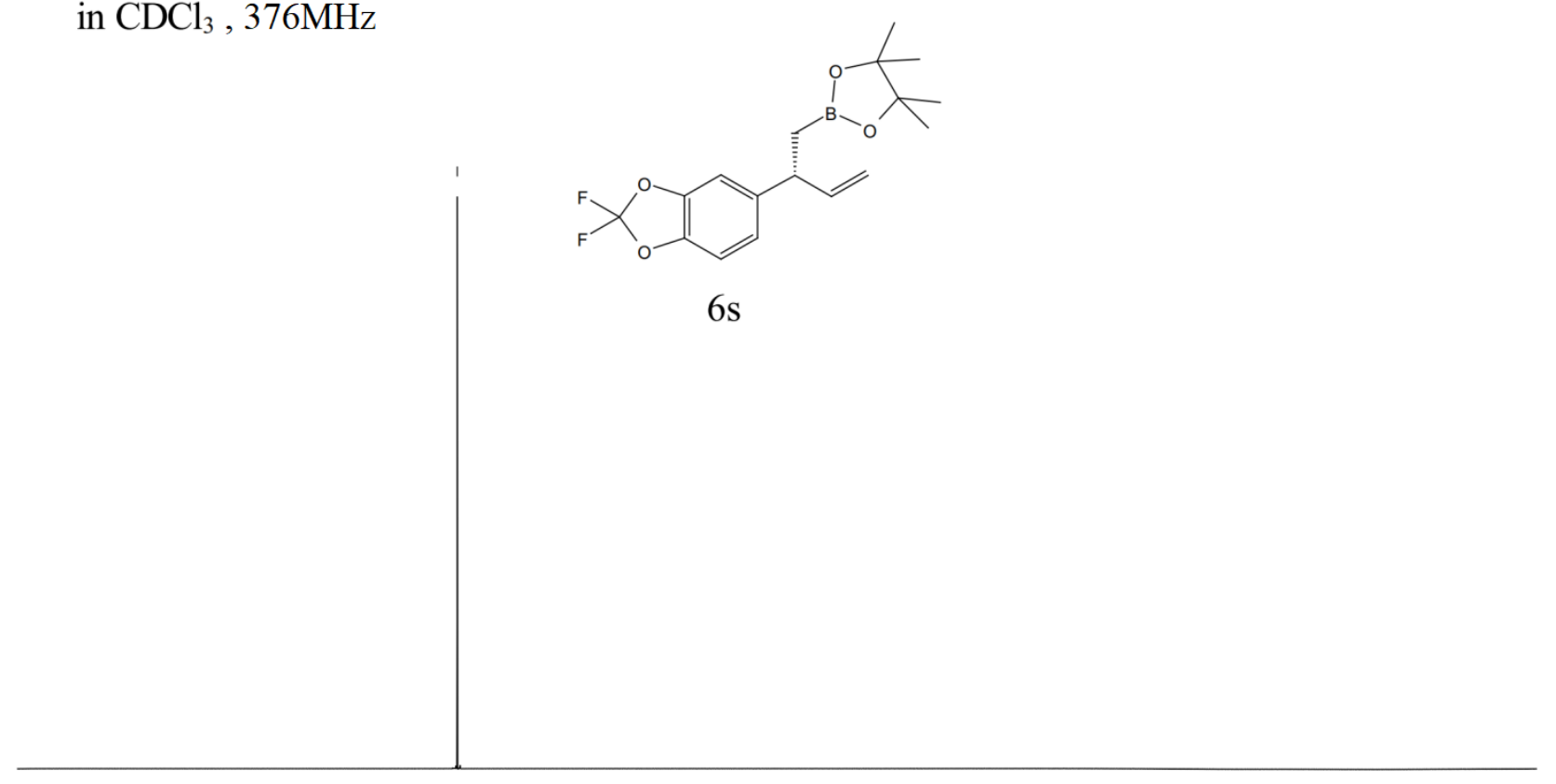

$\begin{array}{lllllllllllllllllllllll}10 & 0 & -10 & -20 & -30 & -40 & -50 & -60 & -70 & -80 & -90 & -100 & -110 & -120 & -130 & -140 & -150 & -160 & -170 & -180 & -190 & -200 & -210\end{array}$ 
in $\mathrm{CDCl}_{3}, 400 \mathrm{MHz}$

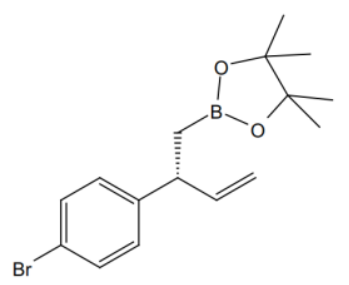

$6 b$
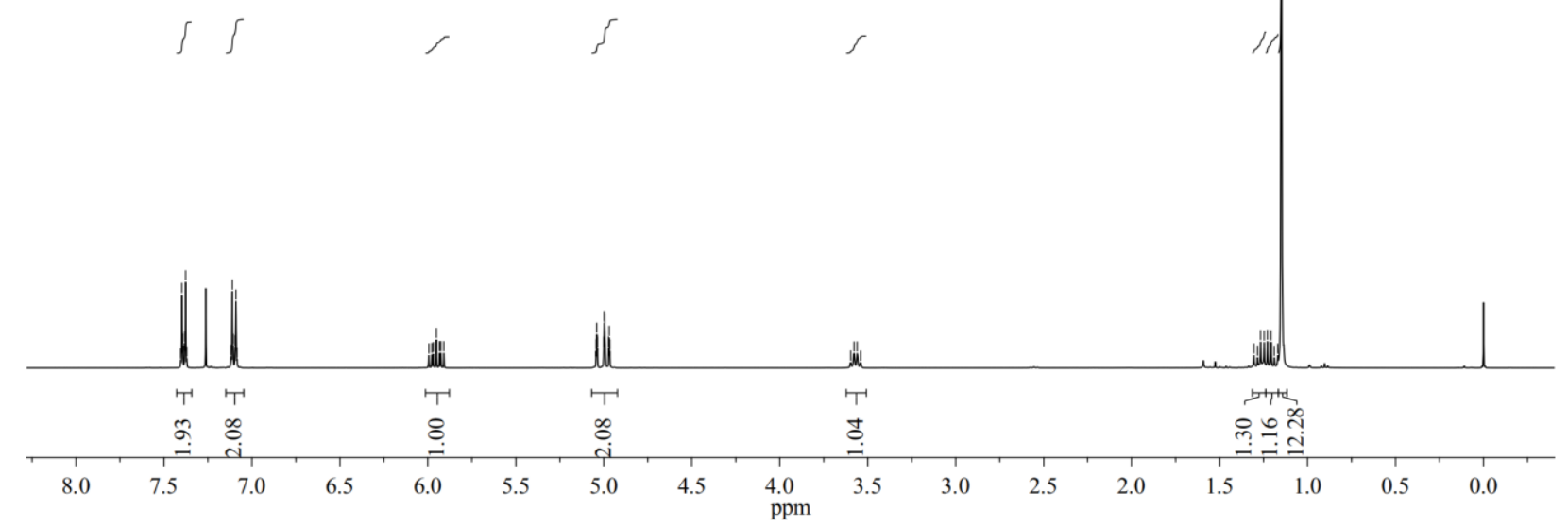

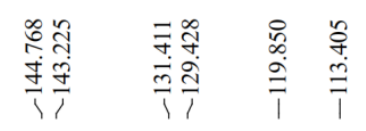

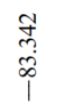

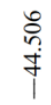

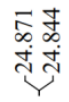

in $\mathrm{CDCl}_{3}, 101 \mathrm{MHz}$

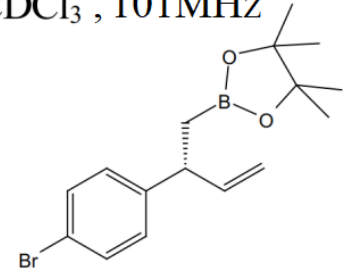

$6 \mathrm{~b}$
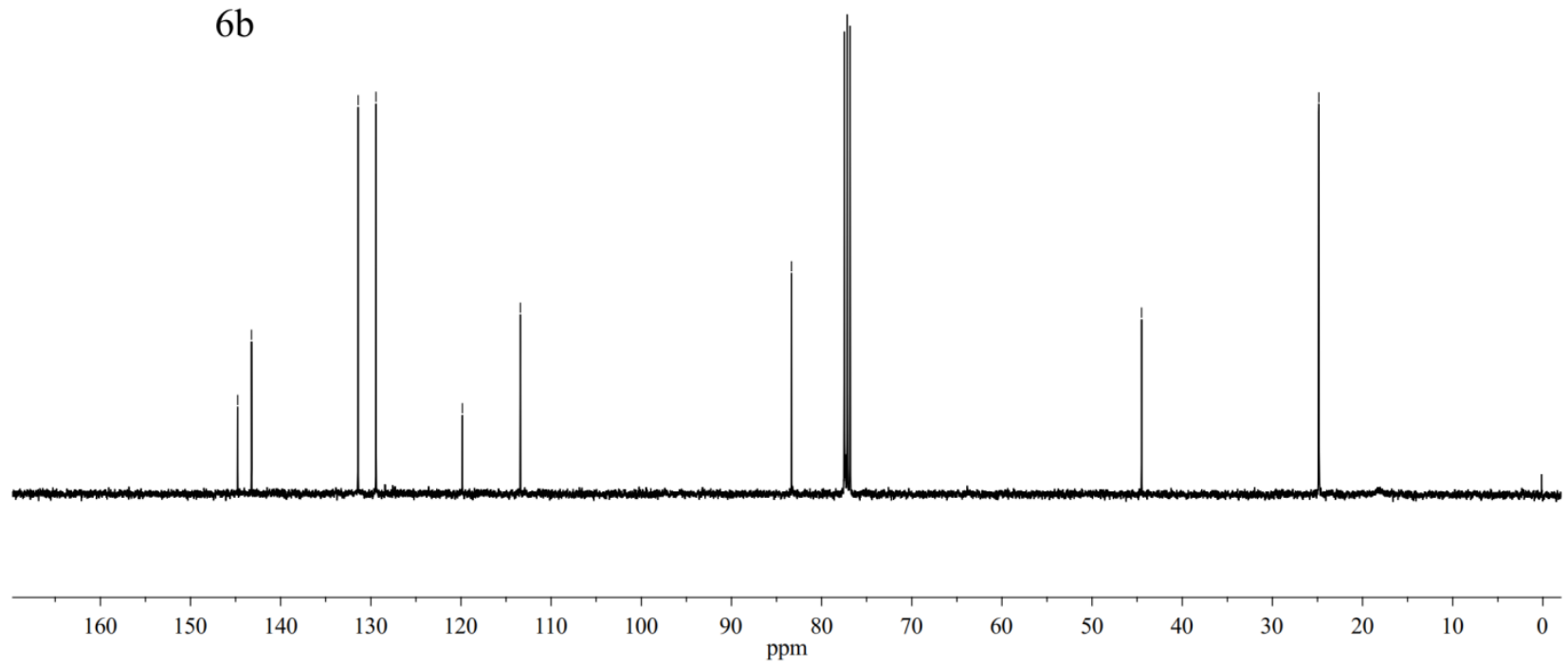
in $\mathrm{CDCl}_{3}, 400 \mathrm{MHz}$<smiles>C=CC(CB1OC(C)(C)C(C)(C)O1)c1cccc(Br)c1</smiles>

s/ $\int$

$6 \mathrm{c}$

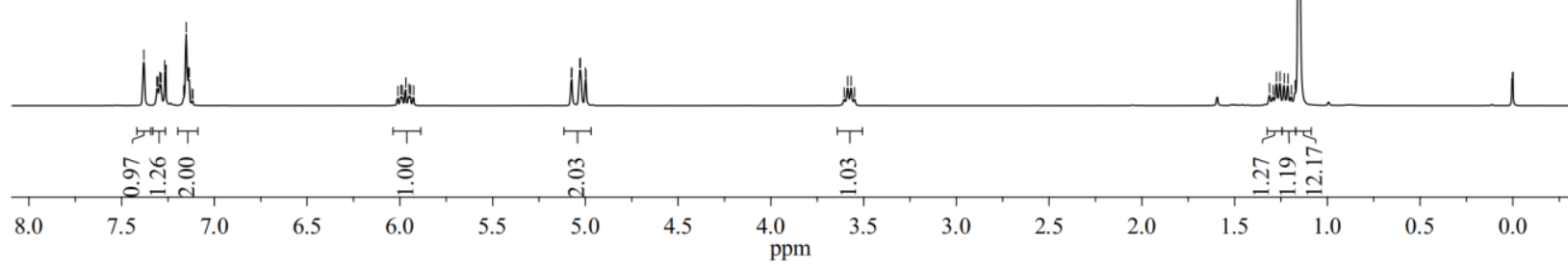

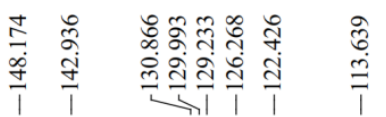

$\underset{\substack{+\infty}}{\infty}$

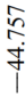

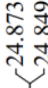

in $\mathrm{CDCl}_{3}, 101 \mathrm{MHz}$<smiles>C=CC(CB1OC(C)(C)C(C)(C)O1)c1cccc(Br)c1</smiles>

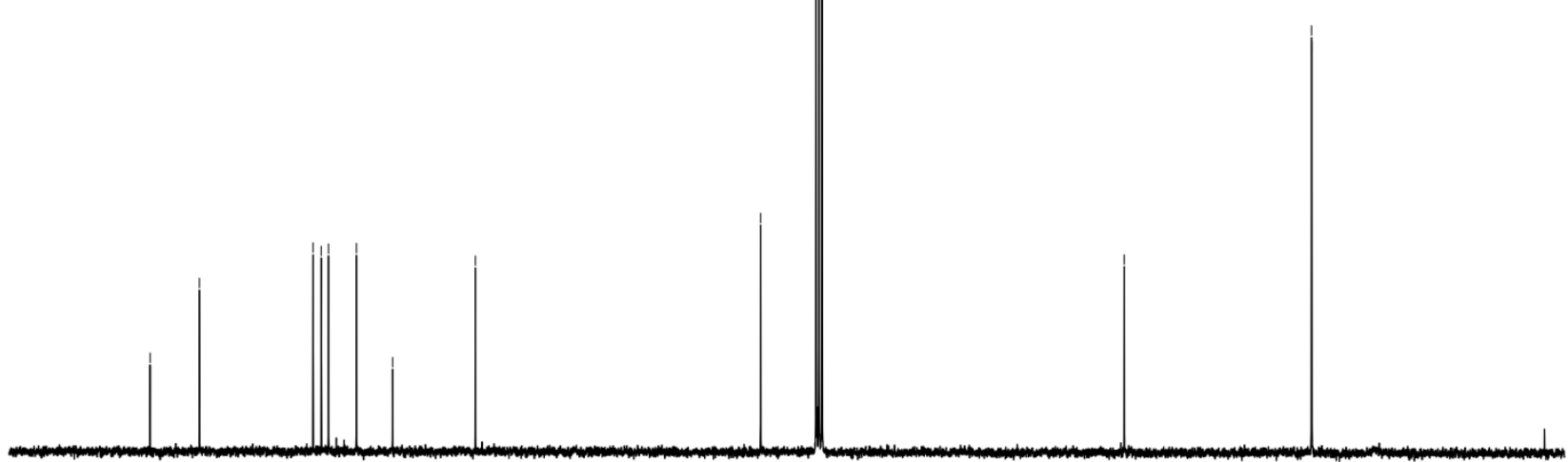

$\begin{array}{lllllllll}160 & 150 & 140 & 130 & 120 & 110 & 100 & 90 & 80\end{array}$ 

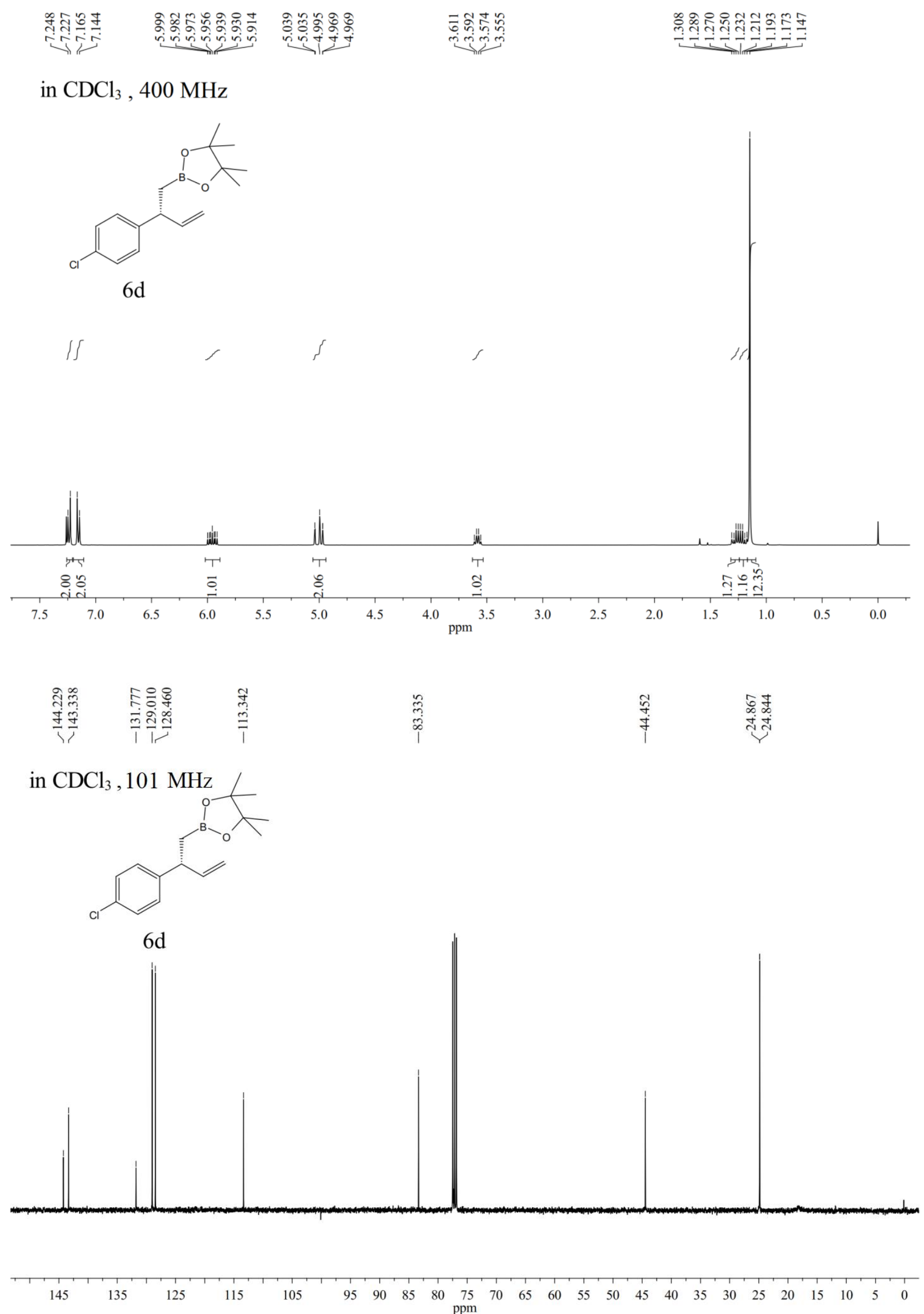


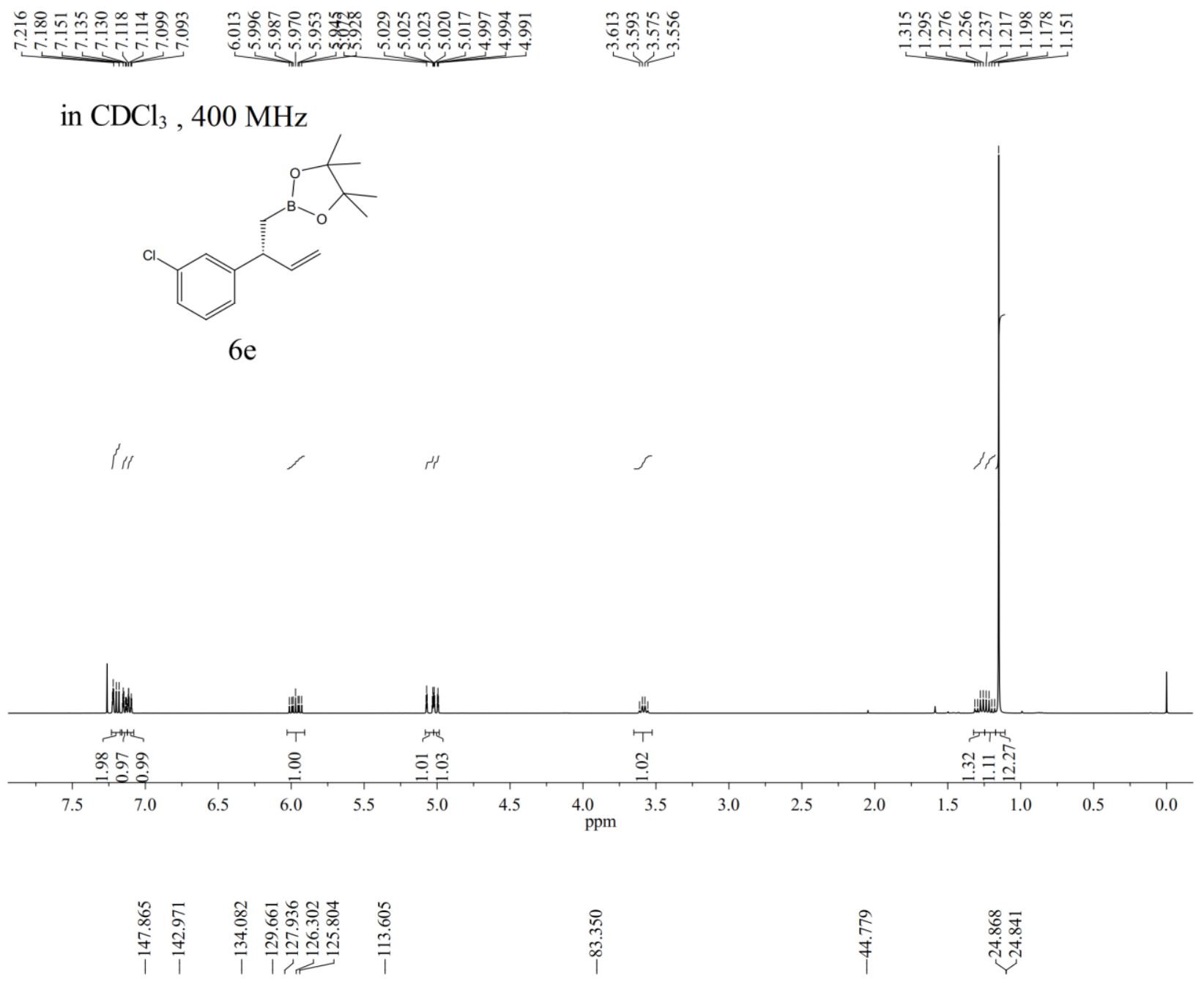

in $\mathrm{CDCl}_{3}, 101 \mathrm{MHz}$

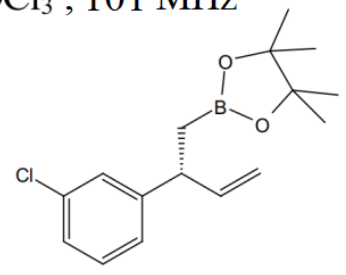

$6 e$

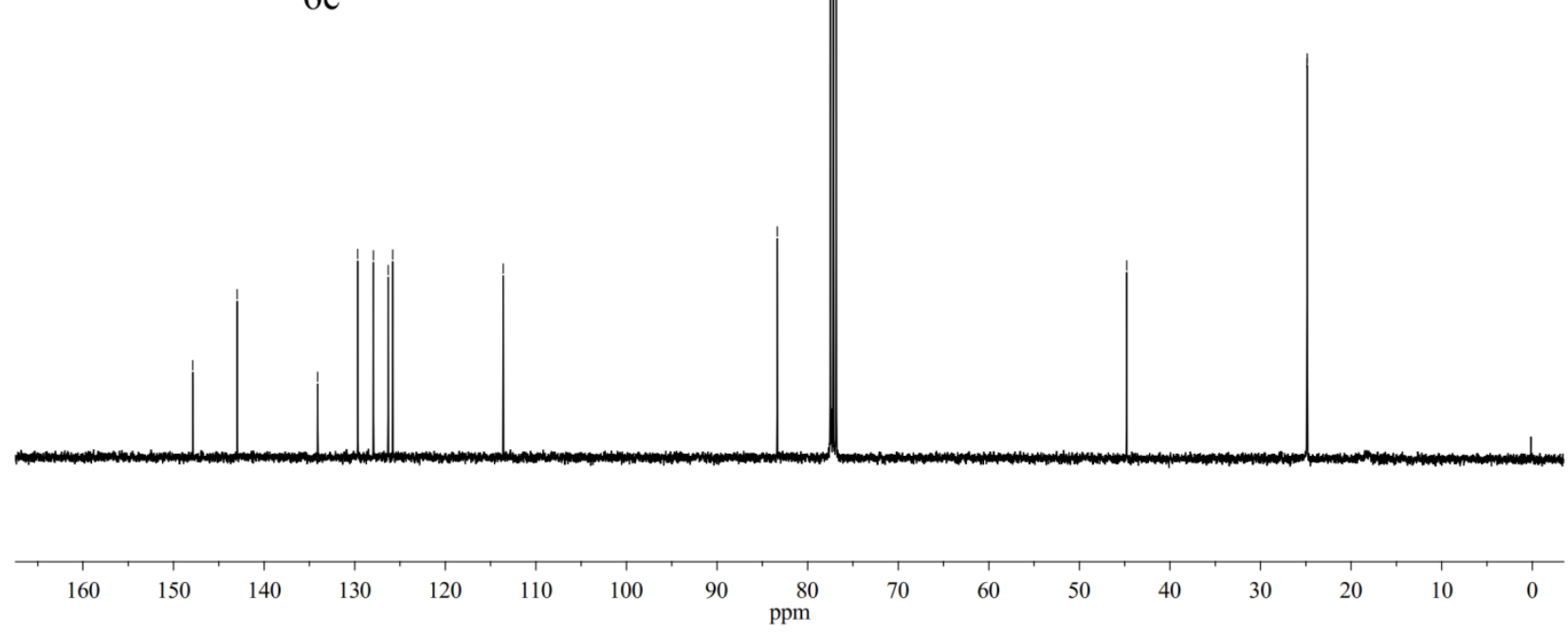




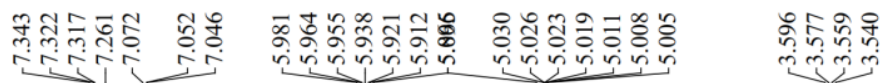

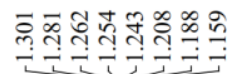

in $\mathrm{CDCl}_{3}, 400 \mathrm{MHz}$
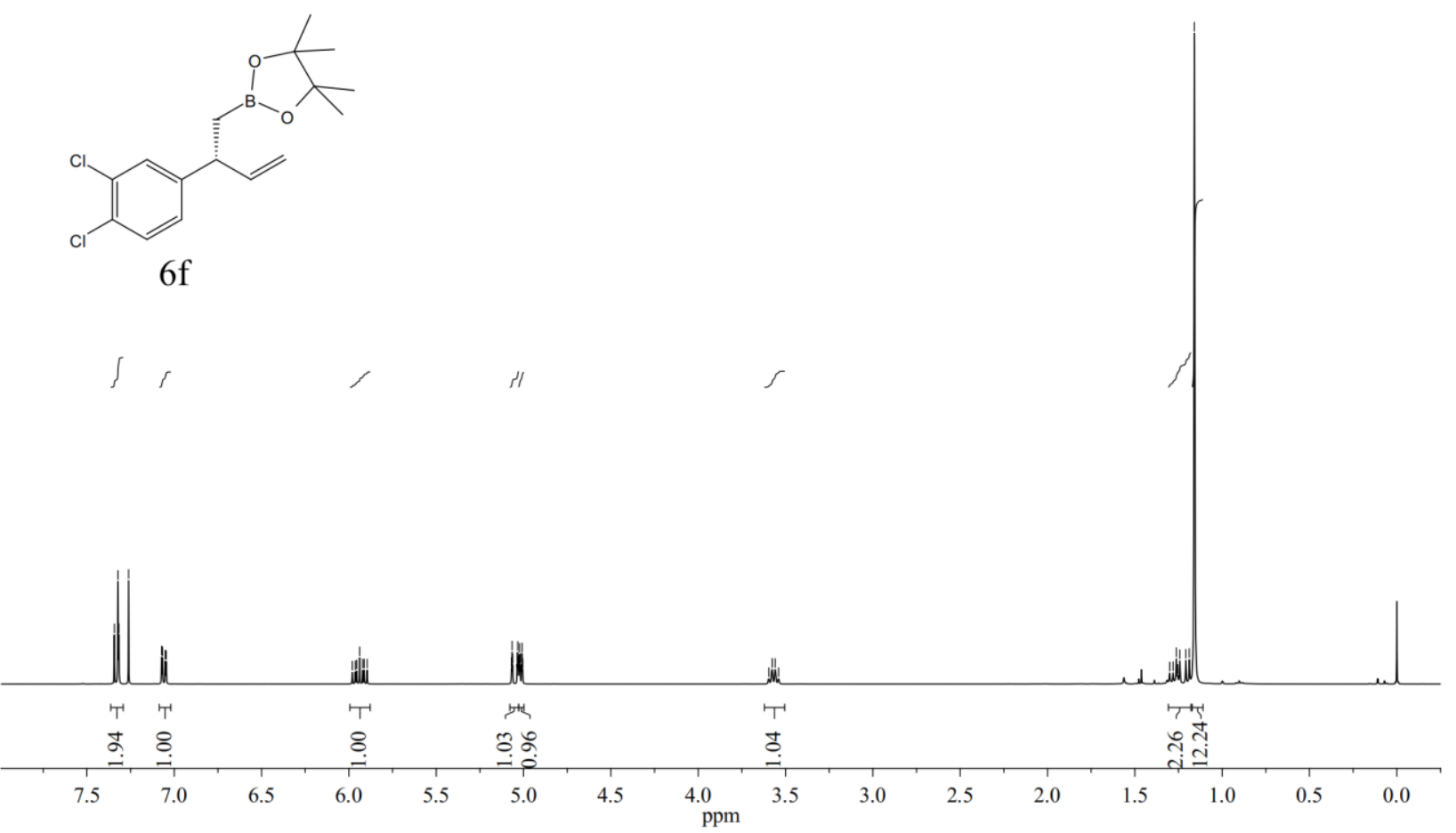

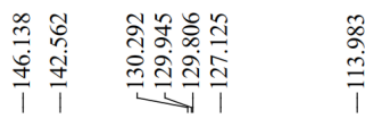

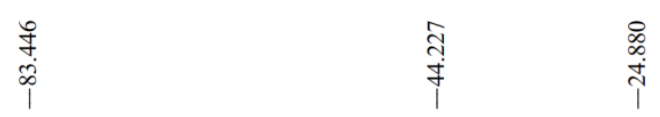

in $\mathrm{CDCl}_{3}, 101 \mathrm{MHz}$

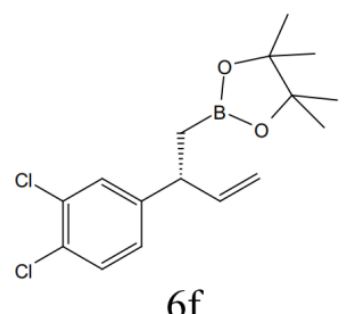

$6 f$
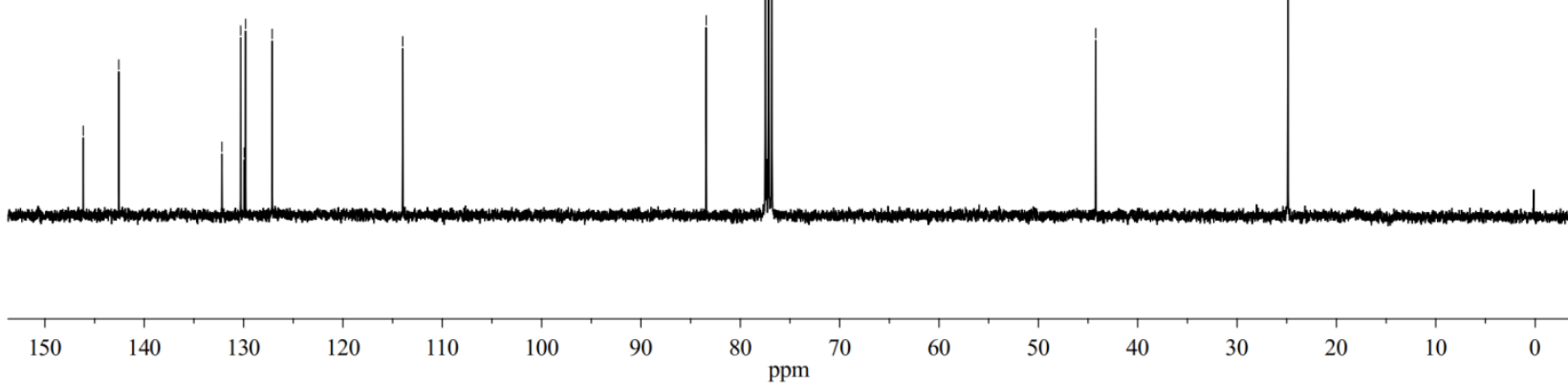


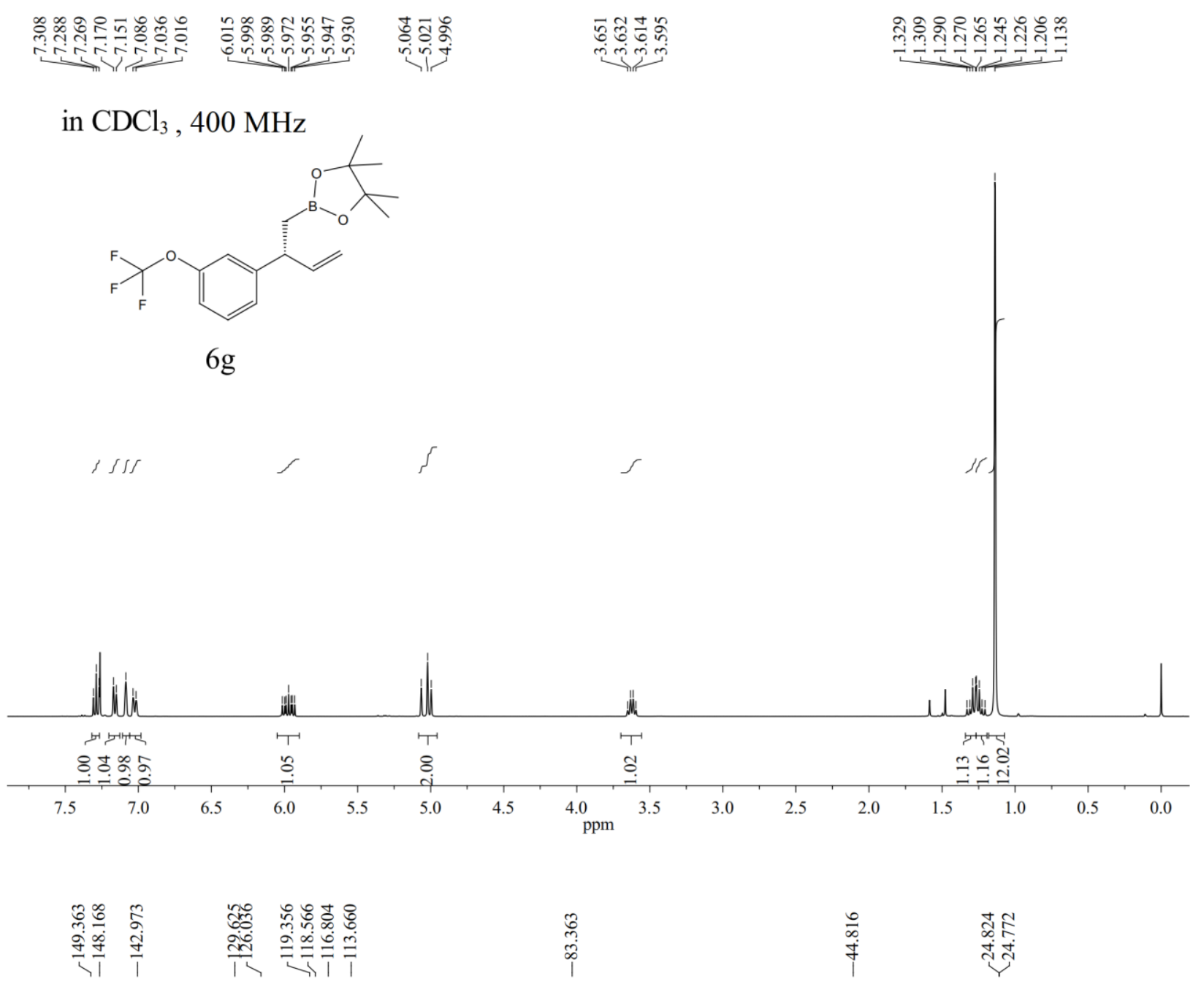

in $\mathrm{CDCl}_{3}, 101 \mathrm{MHz}$
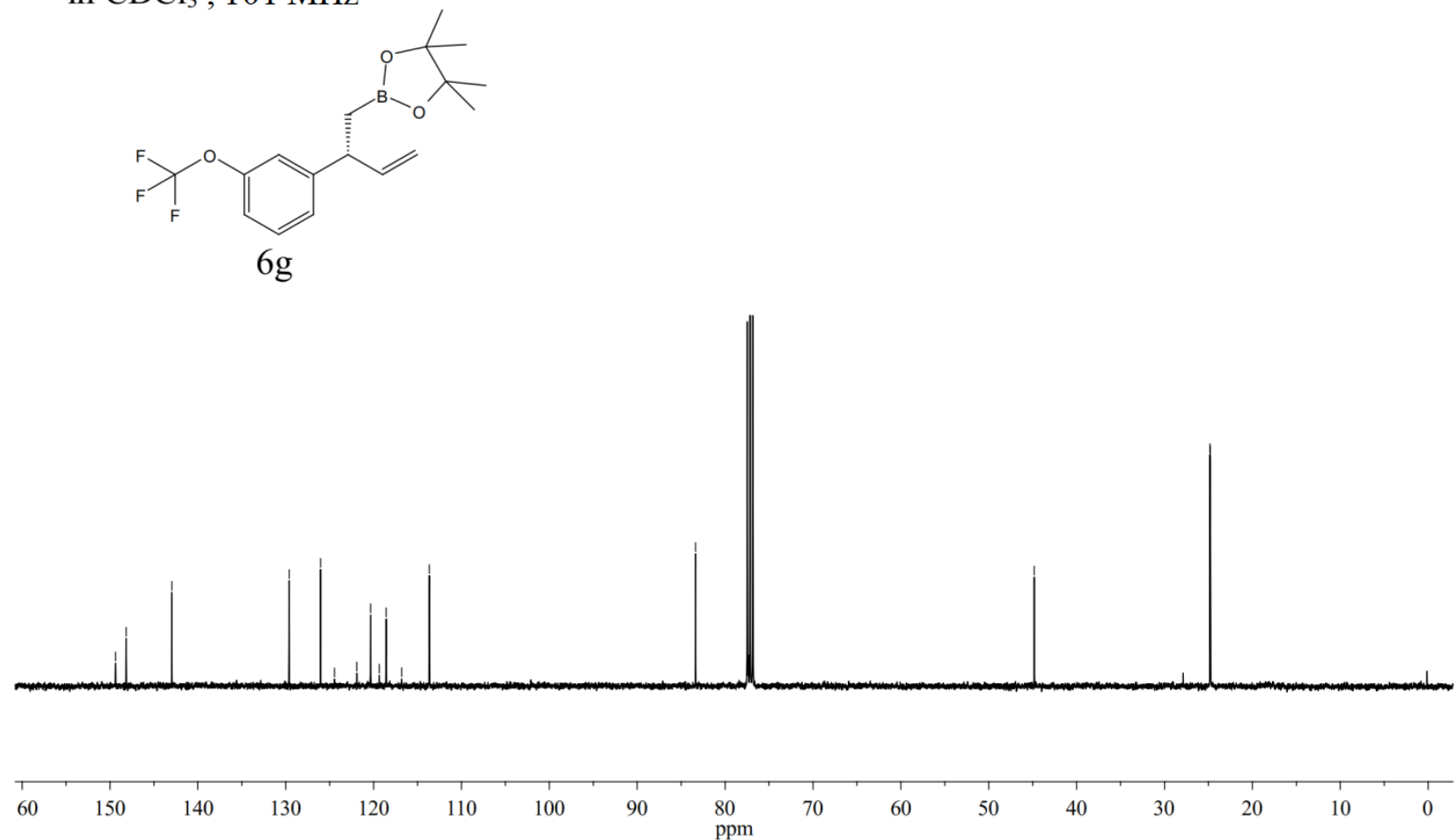


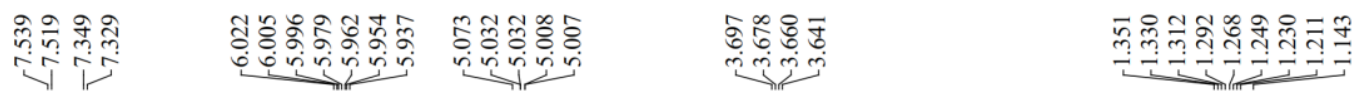

in $\mathrm{CDCl}_{3}, 400 \mathrm{MHz}$

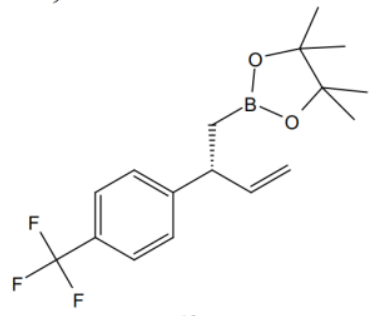

$6 \mathrm{~h}$

$\iint$

Mil

'T 'T

88

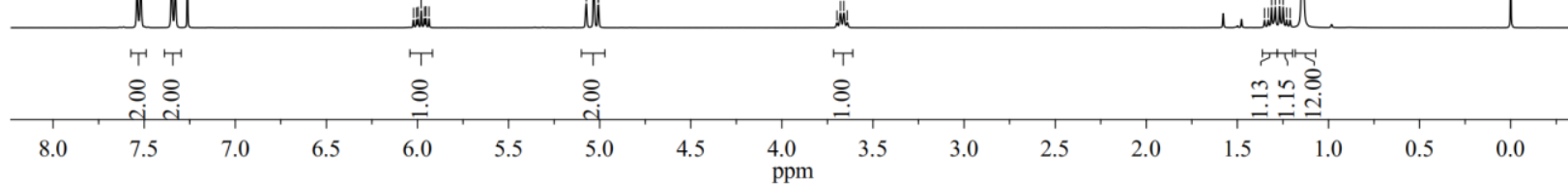

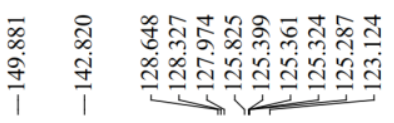

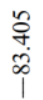

商

닌

in $\mathrm{CDCl}_{3}, 101 \mathrm{MHz}$

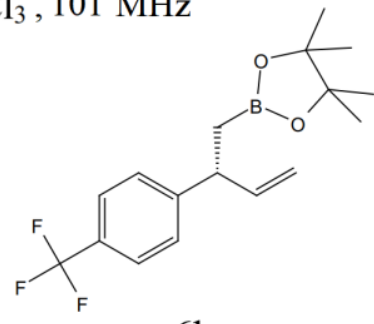

$6 \mathrm{~h}$

$\begin{array}{lllll}160 & 150 & 140 & 130 & 120\end{array}$

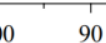

ppm 
in $\mathrm{CDCl}_{3}, 400 \mathrm{MHz}$

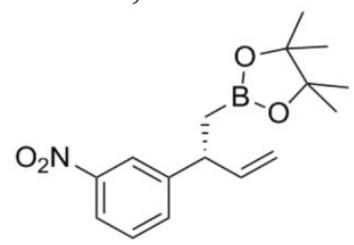

$6 \mathrm{i}$

în

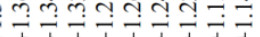

i

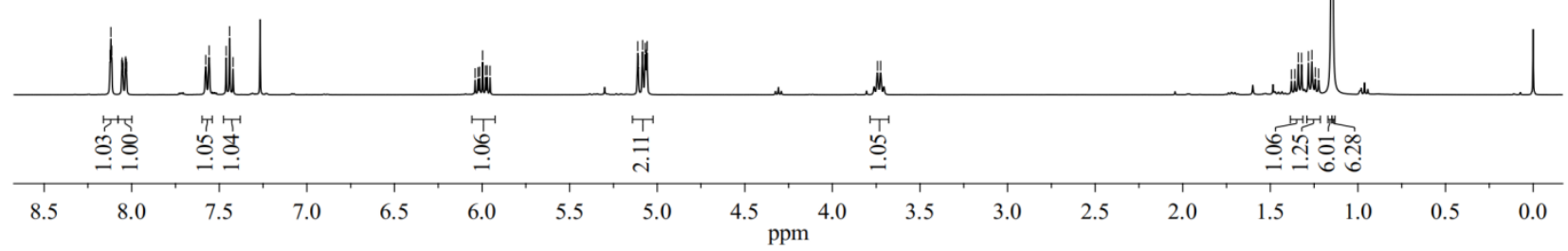

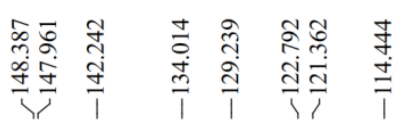

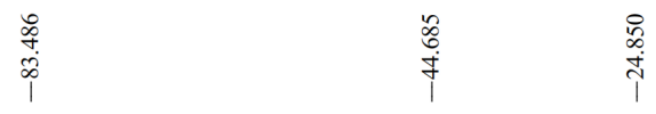

in $\mathrm{CDCl}_{3}, 101 \mathrm{MHz}$<smiles>C=C[C@H](CB1OC(C)(C)C(C)(C)O1)c1cccc([N+](=O)[O-])c1</smiles>

$6 \mathrm{i}$

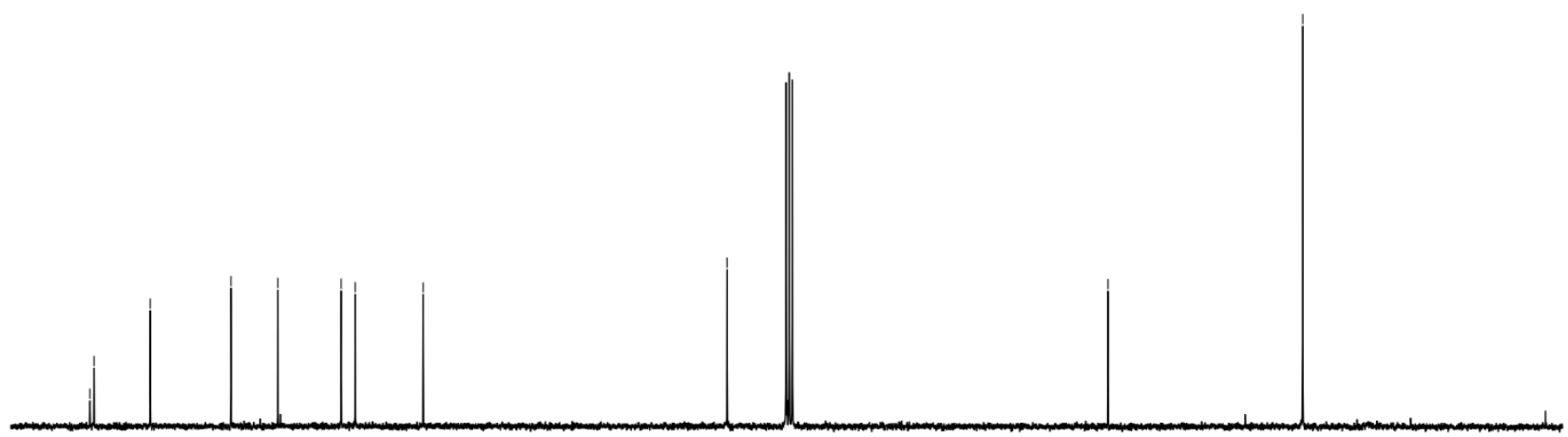

$\begin{array}{llllllllllllll}150 & 140 & 130 & 120 & 110 & 100 & 90 & 80 & 70 & 60 & 50 & 40 & 30 & 20 \\ \text { ppm } & 10 & 0\end{array}$ 


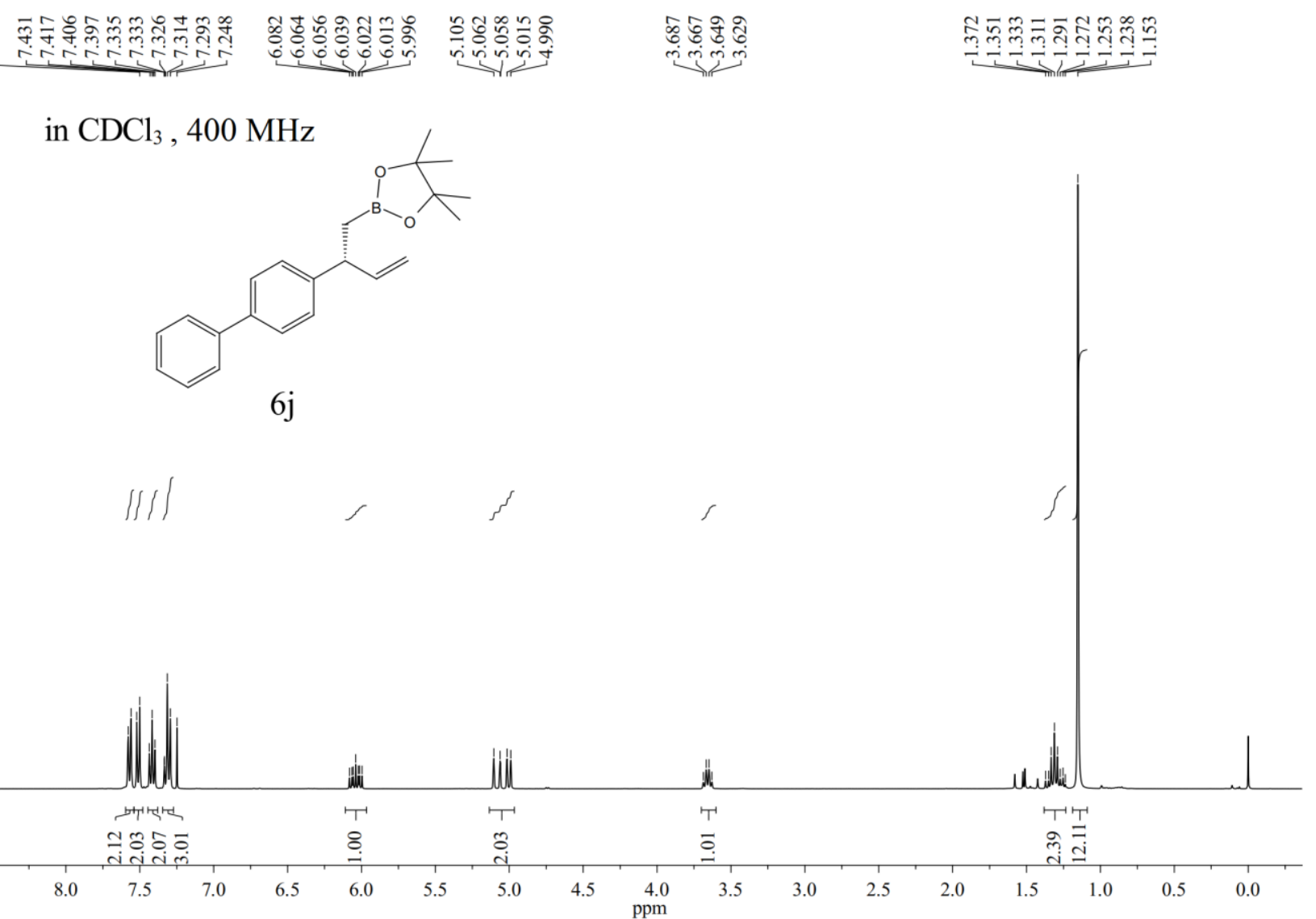

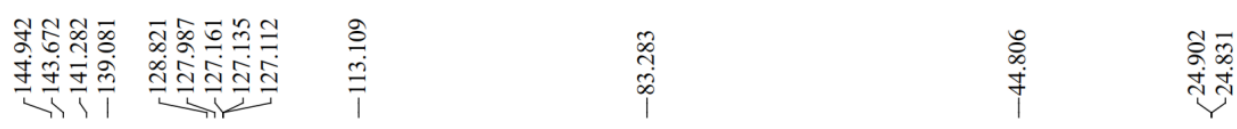

in $\mathrm{CDCl}_{3}, 101 \mathrm{MHz}$
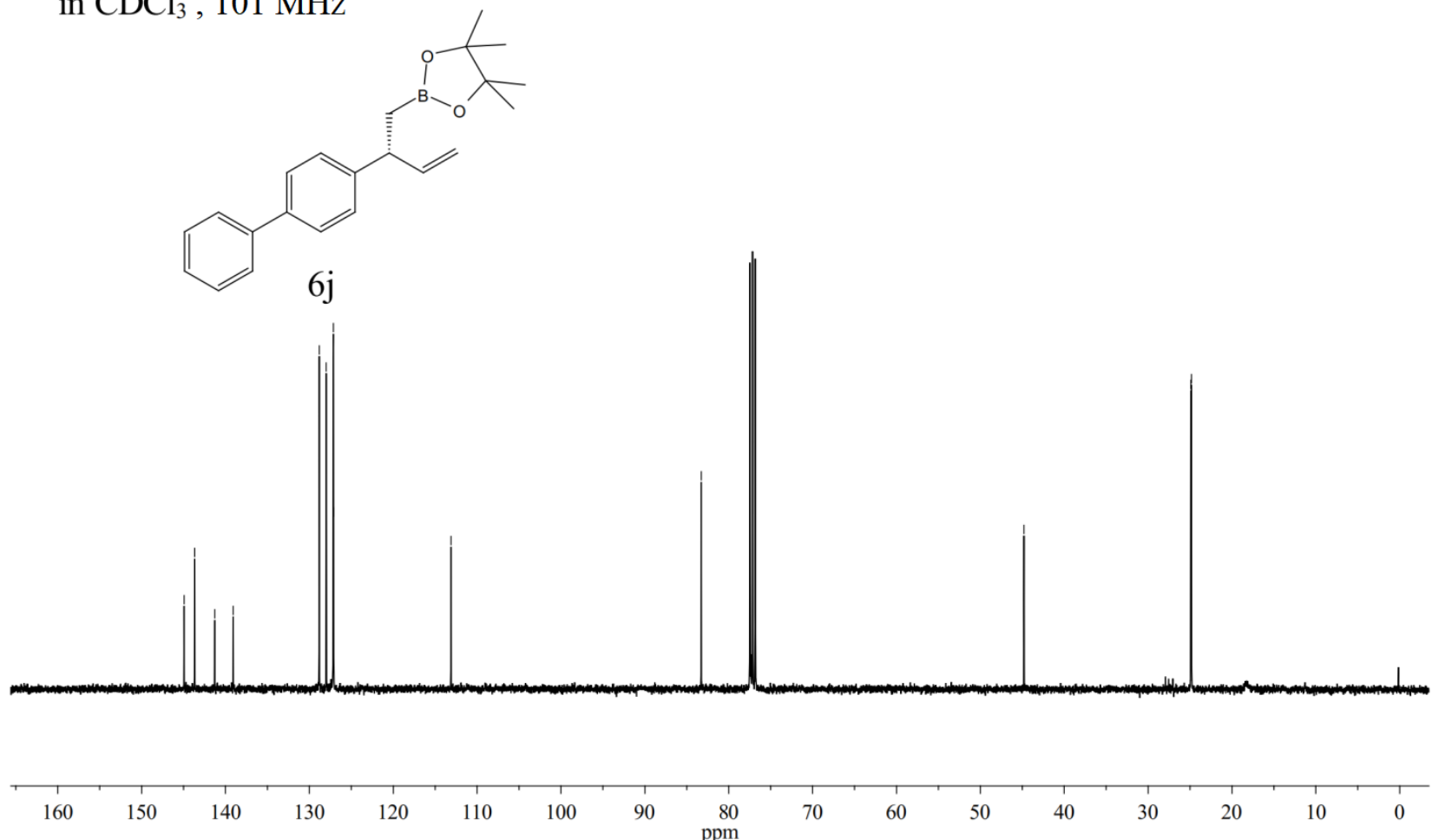


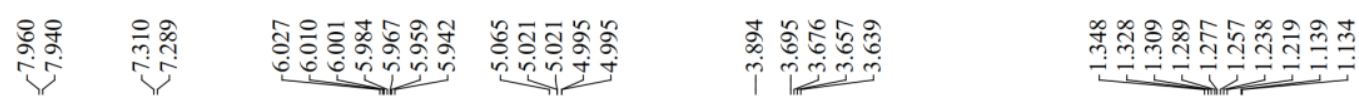

in $\mathrm{CDCl}_{3}, 400 \mathrm{MHz}$

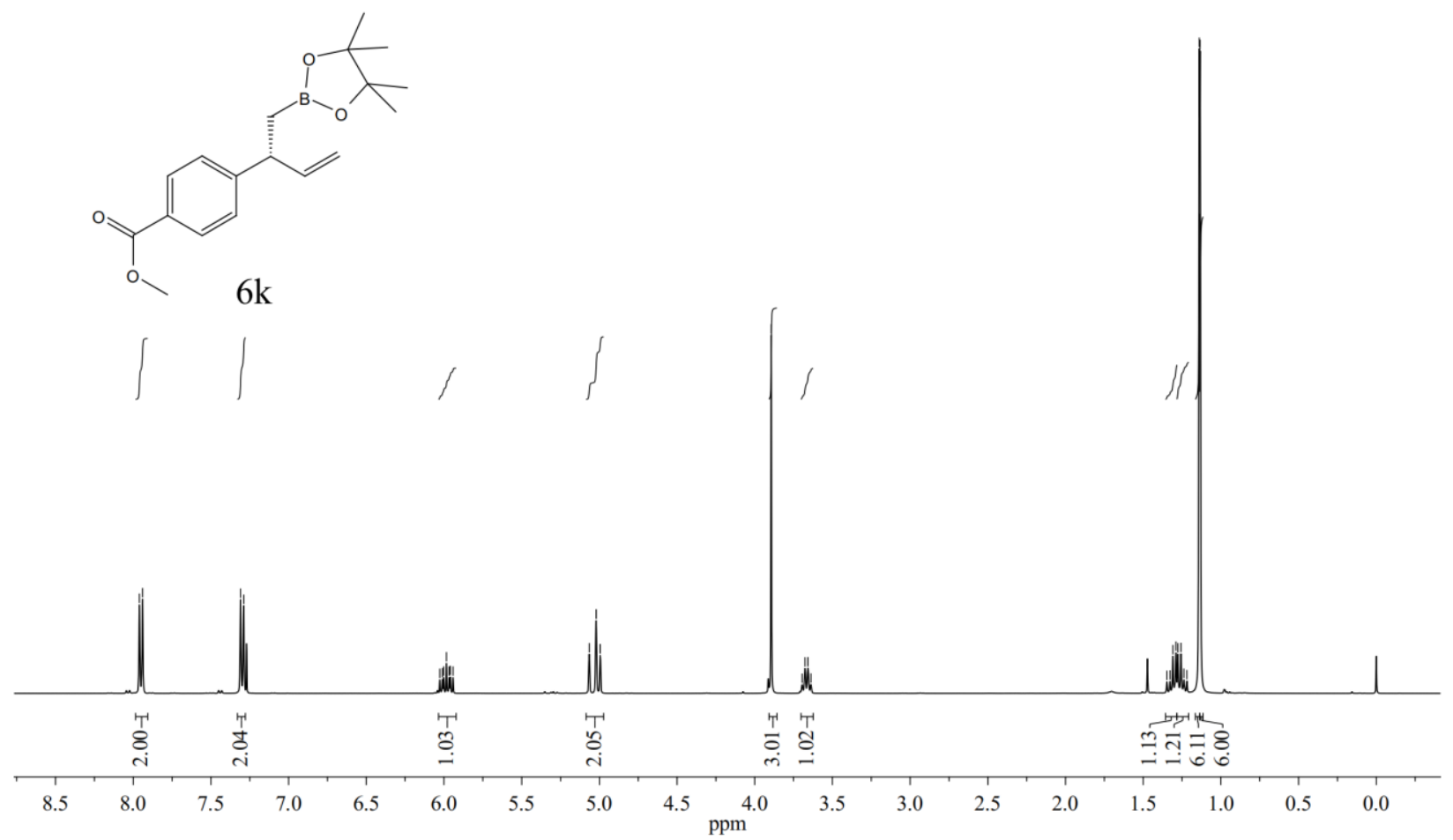

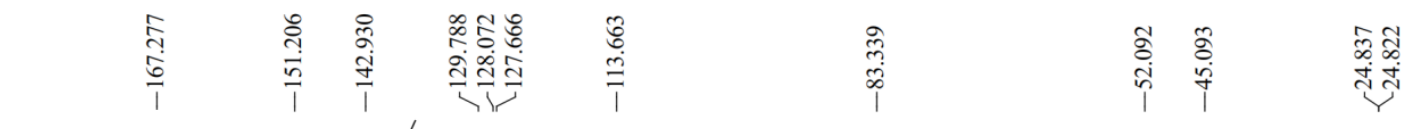

in $\mathrm{CDCl}_{3}, 101 \mathrm{MHz}$
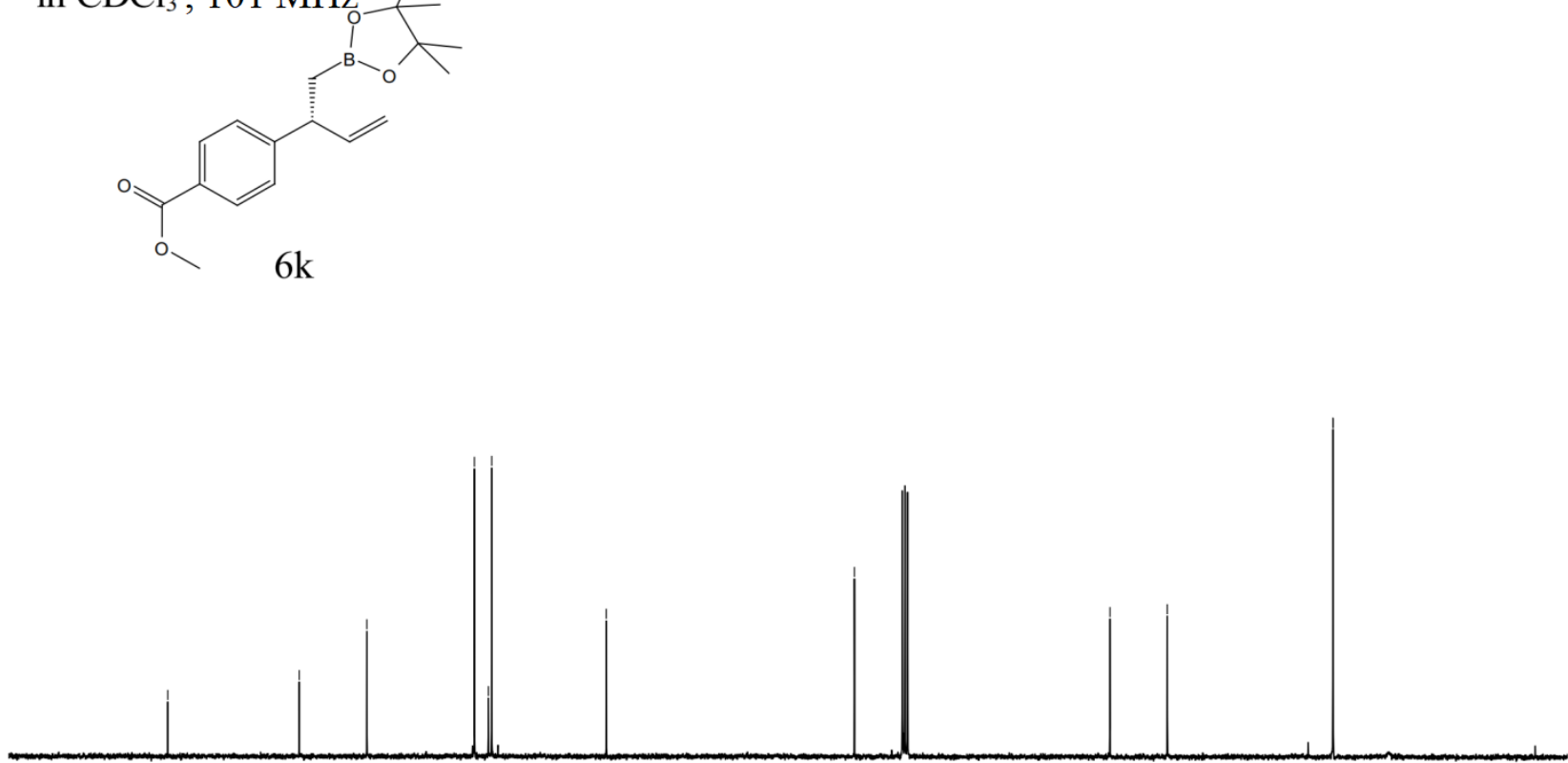

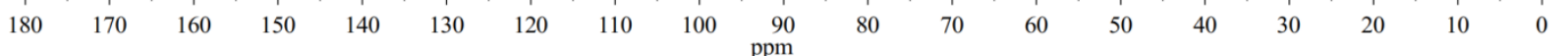


in $\mathrm{CDCl}_{3}, 400 \mathrm{MHz}$

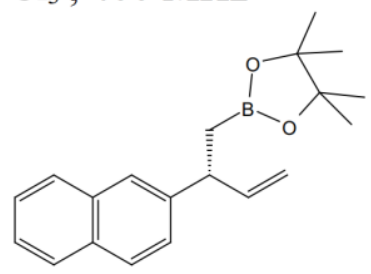

61<smiles>[C]1[CH]CC1</smiles>

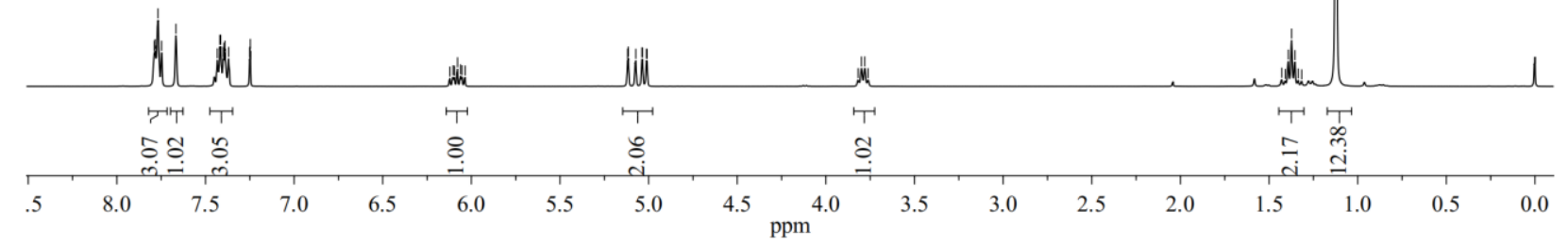

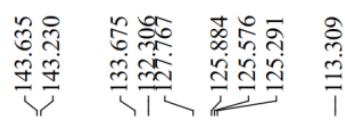

ले
ले
1

年年

in $\mathrm{CDCl}_{3}, 101 \mathrm{MHz}$

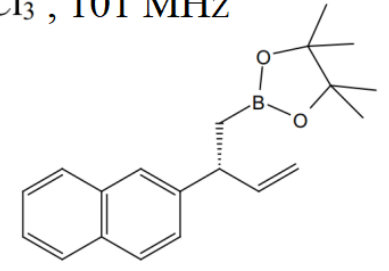

61
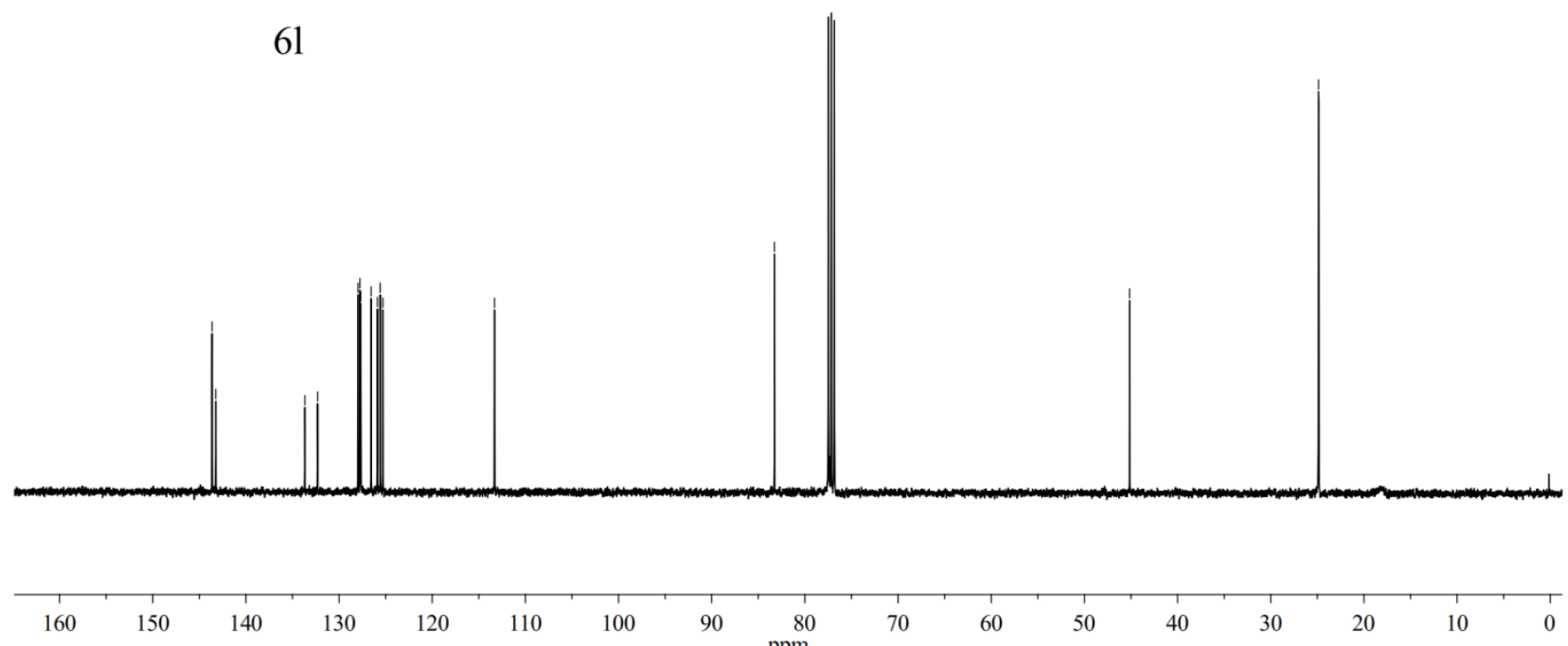

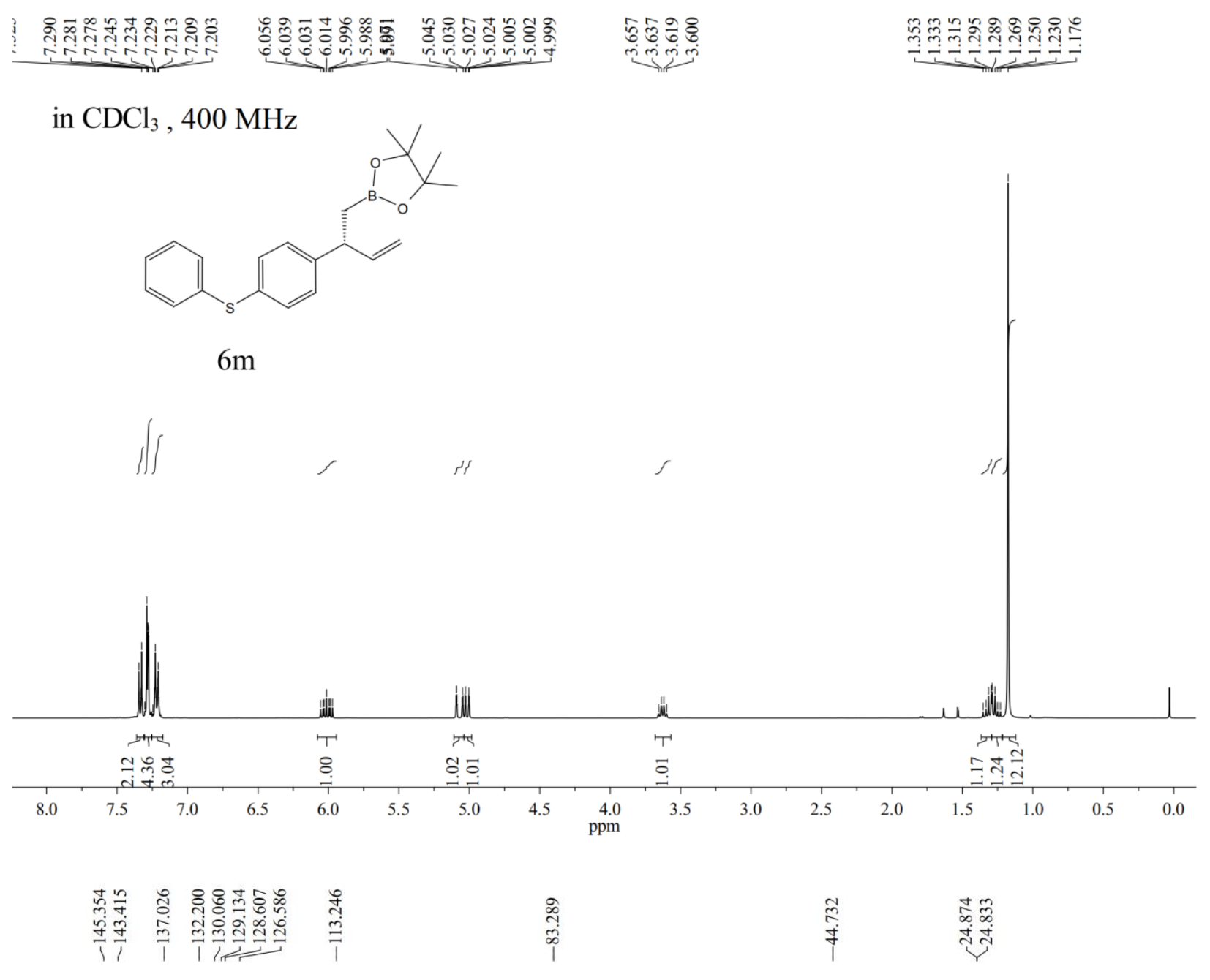

in $\mathrm{CDCl}_{3}, 101 \mathrm{MHz}$
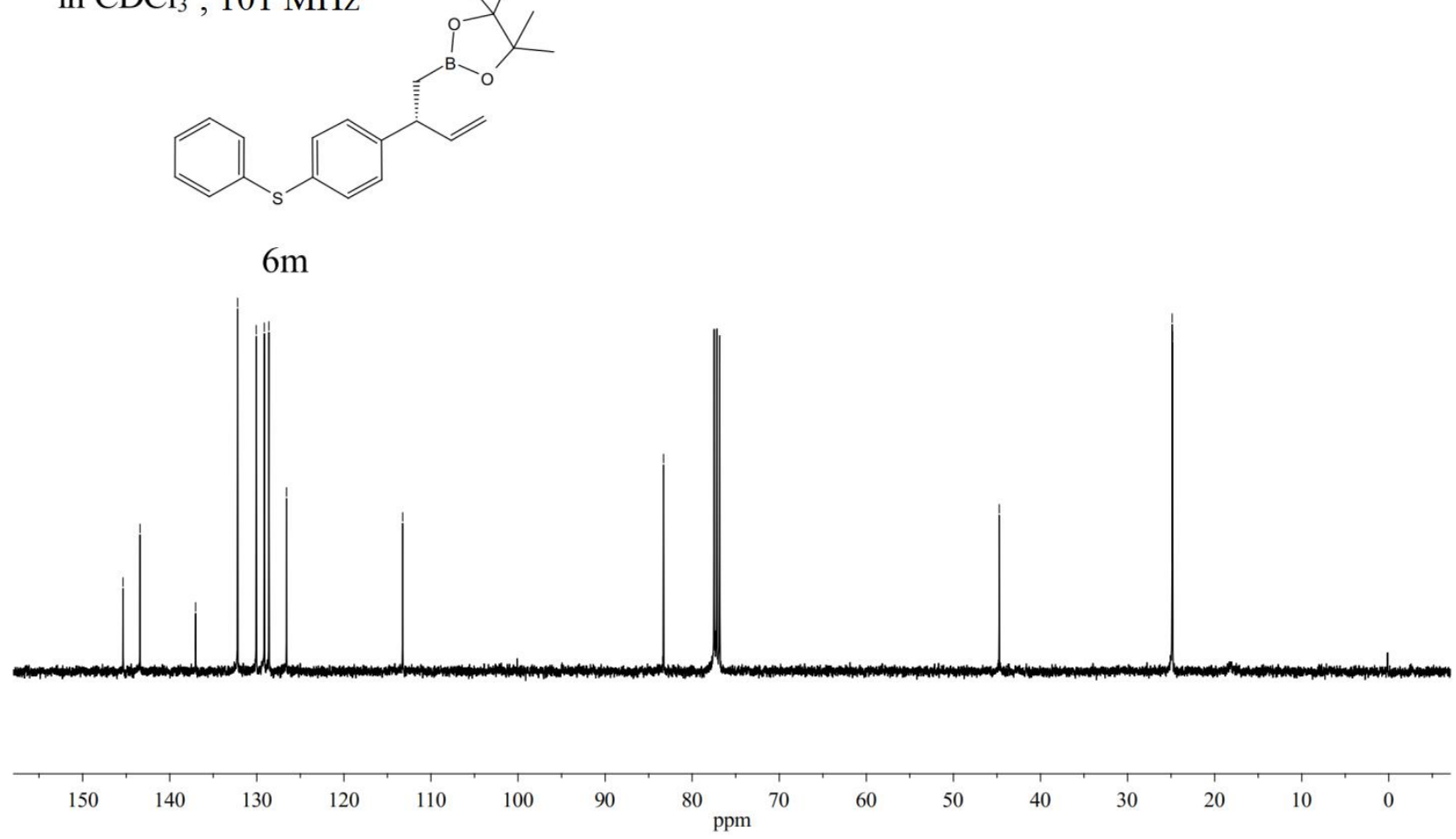


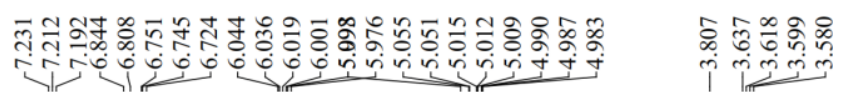

in $\mathrm{CDCl}_{3}, 400 \mathrm{MHz}$
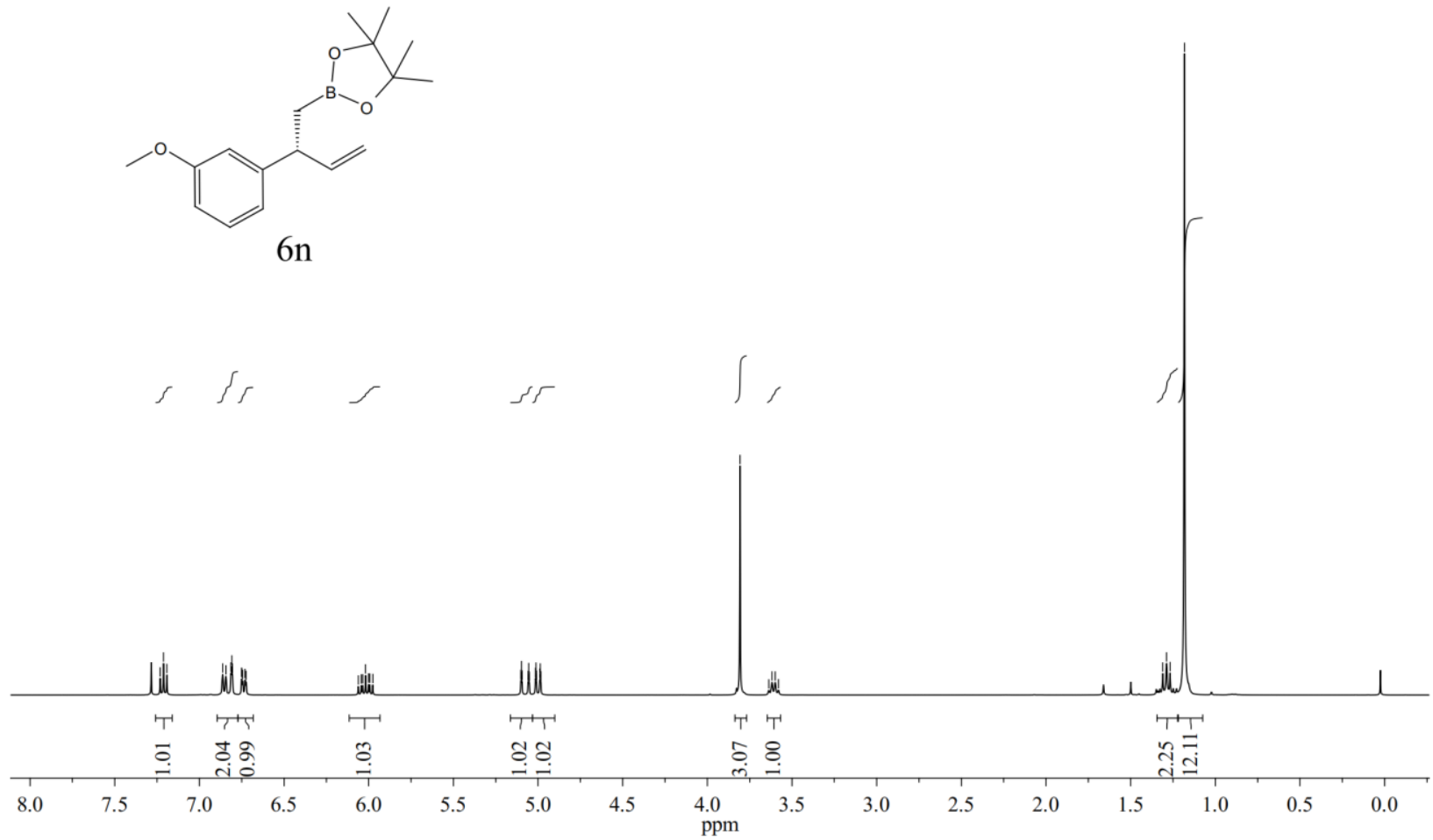

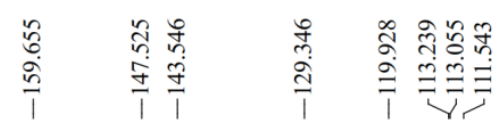

तิ

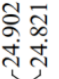

in $\mathrm{CDCl}_{3}, 101 \mathrm{MHz}$
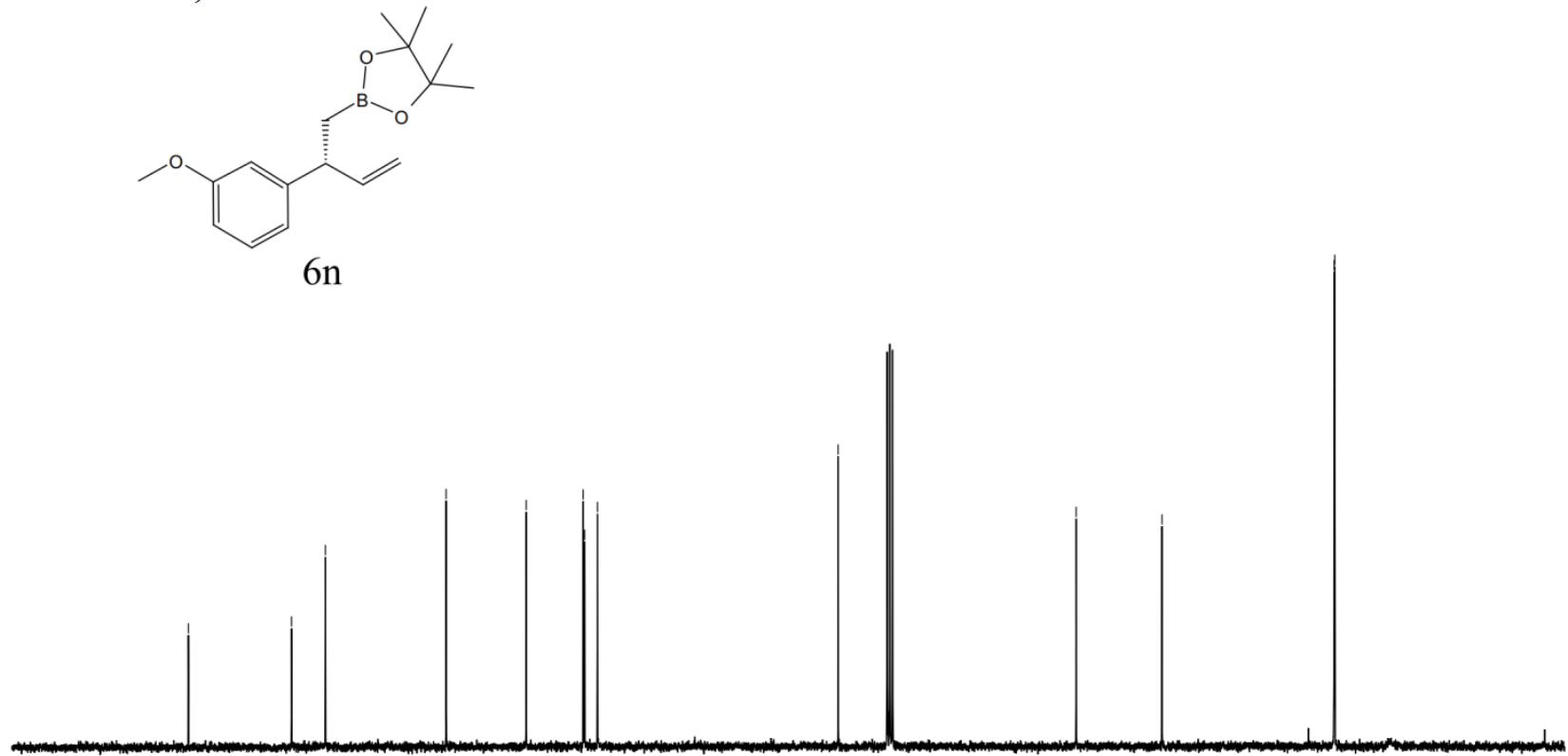

$\begin{array}{lllllllllllllllllll}80 & 170 & 160 & 150 & 140 & 130 & 120 & 110 & 100 & 90 & 80 & 70 & 60 & 50 & 40 & 30 & 20 & 10 & 0\end{array}$ 


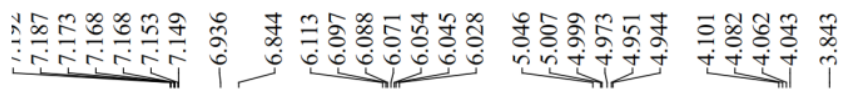

in $\mathrm{CDCl}_{3}, 400 \mathrm{MHz}$

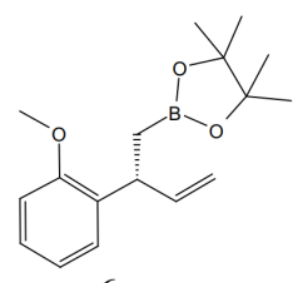

60<smiles>C1CCCCCC1</smiles>

$\left.\int\right\}$

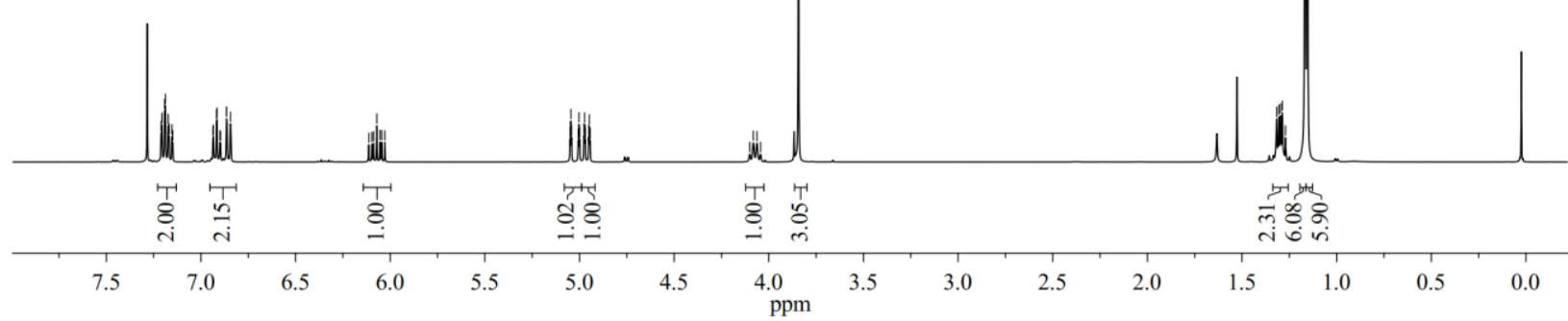

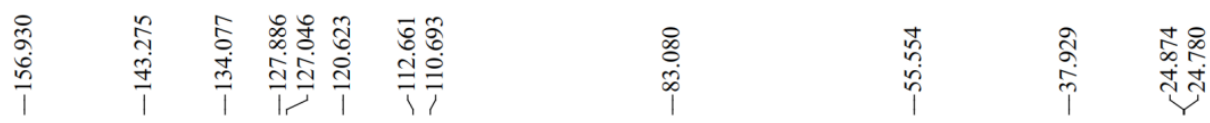

in $\mathrm{CDCl}_{3}, 101 \mathrm{MHz}$

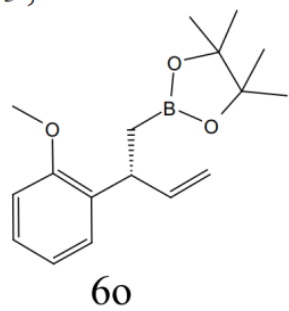

60

$\begin{array}{llllllll}170 & 160 & 150 & 140 & 130 & 120 & 110 & 100\end{array}$

ppm $^{80}$ 


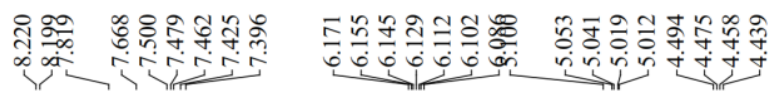

in $\mathrm{CDCl}_{3} .400 \mathrm{MHz}$

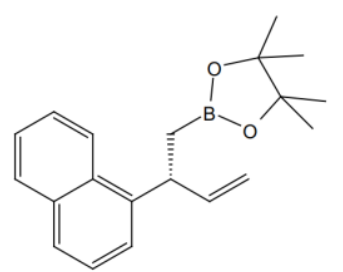

$6 \mathrm{p}$

111

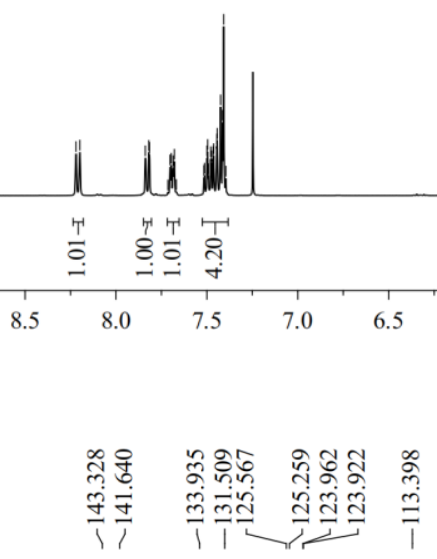

in $\mathrm{CDCl}_{3}, 101 \mathrm{MHz}$

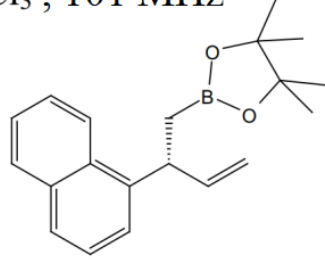

$6 p$

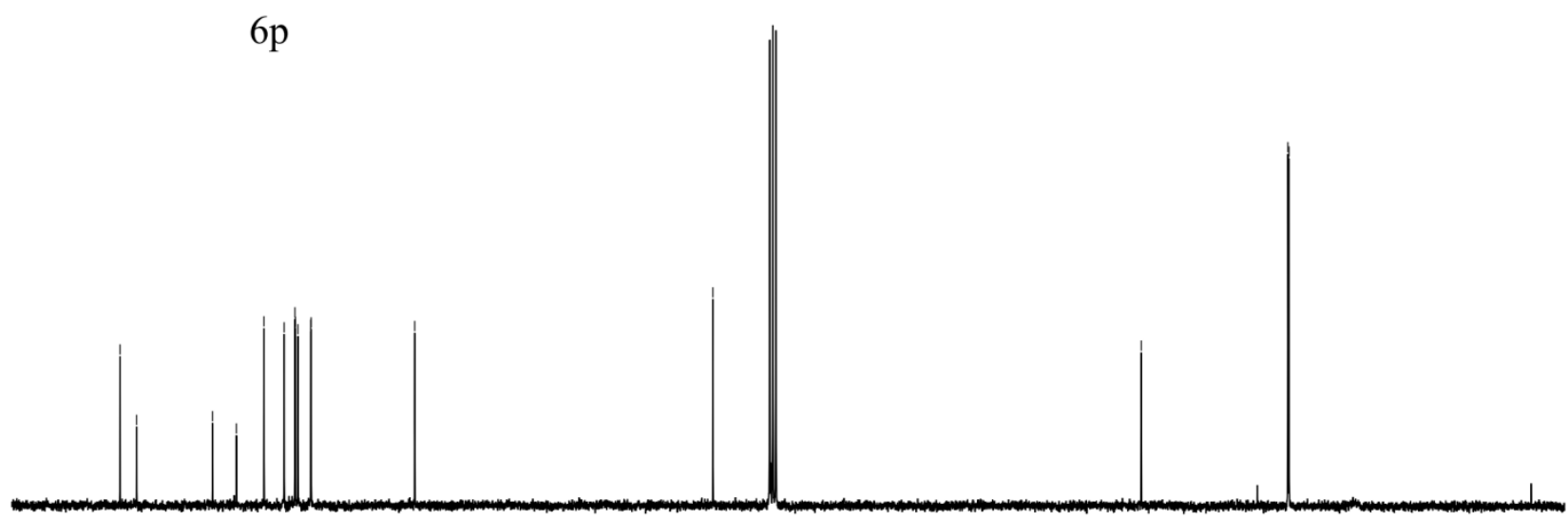

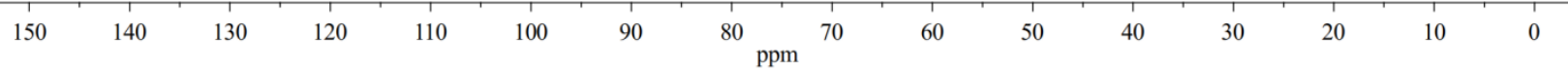



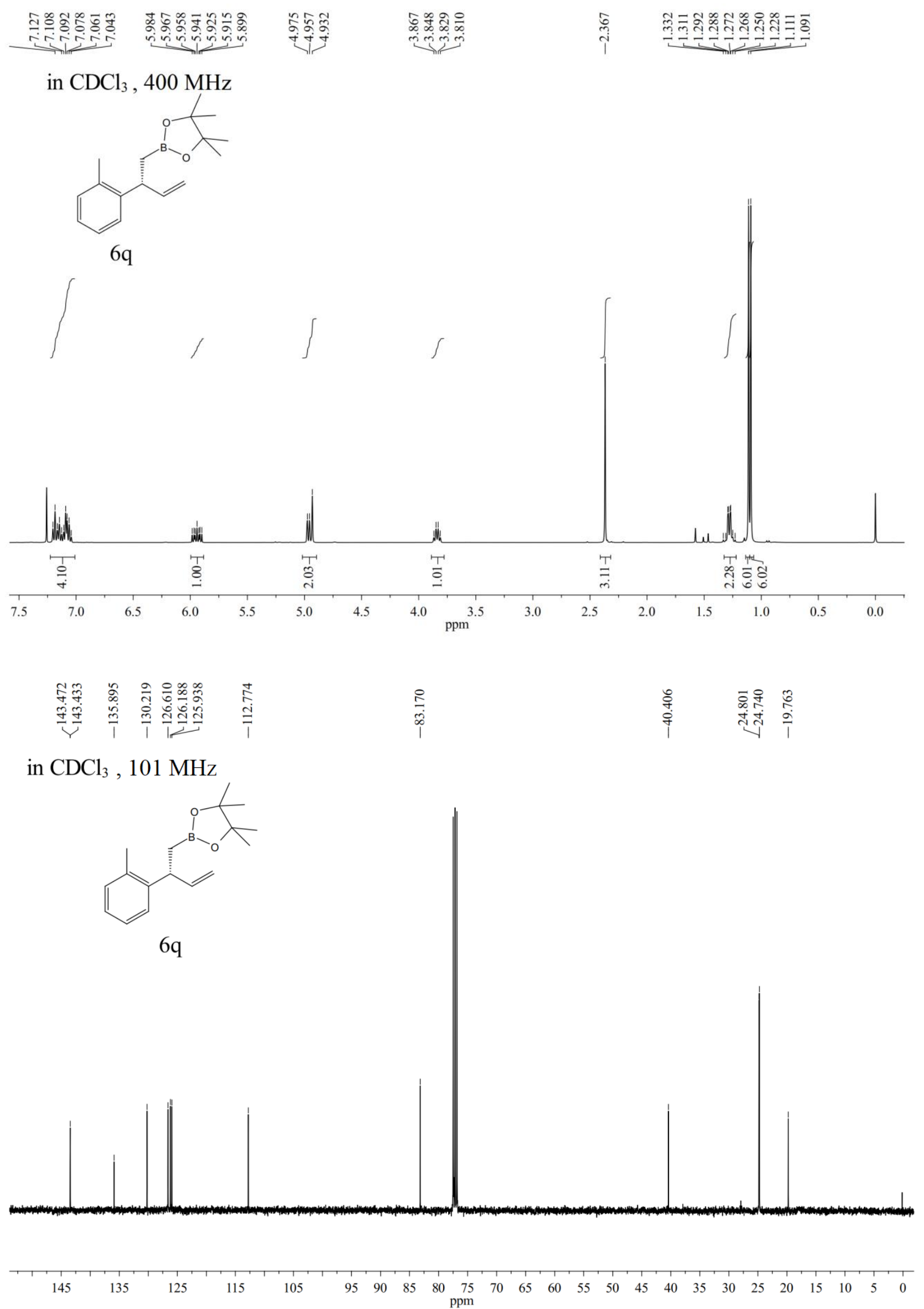


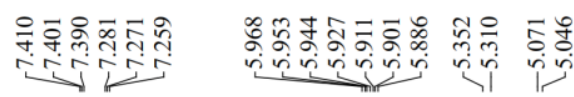

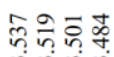

लिखंल

ลี่

in $\mathrm{CDCl}_{3}, 400 \mathrm{MHz}$

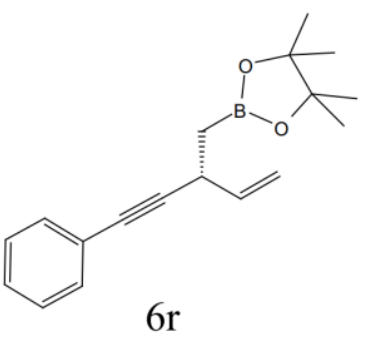

$\int$

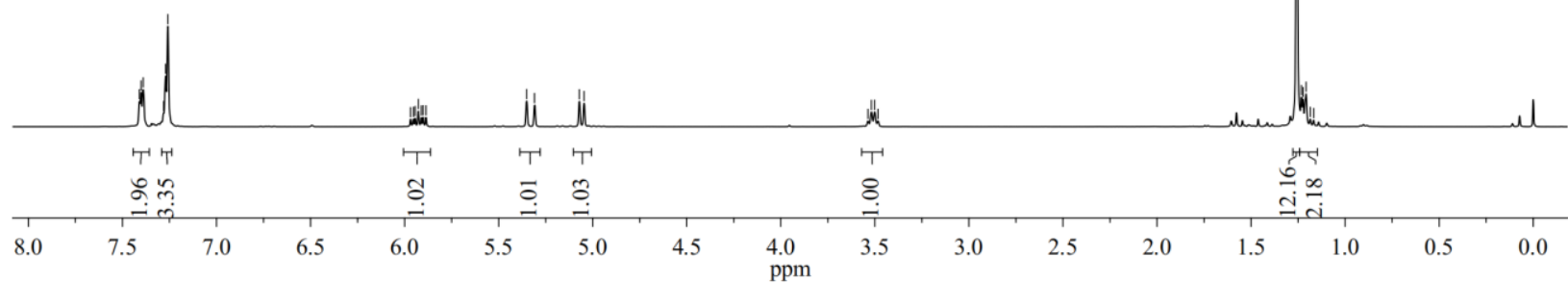

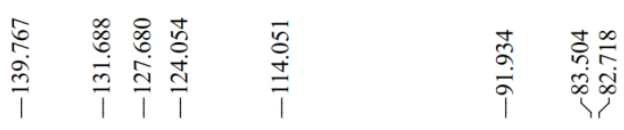

in $\mathrm{CDCl}_{3}, 101 \mathrm{MHz}$
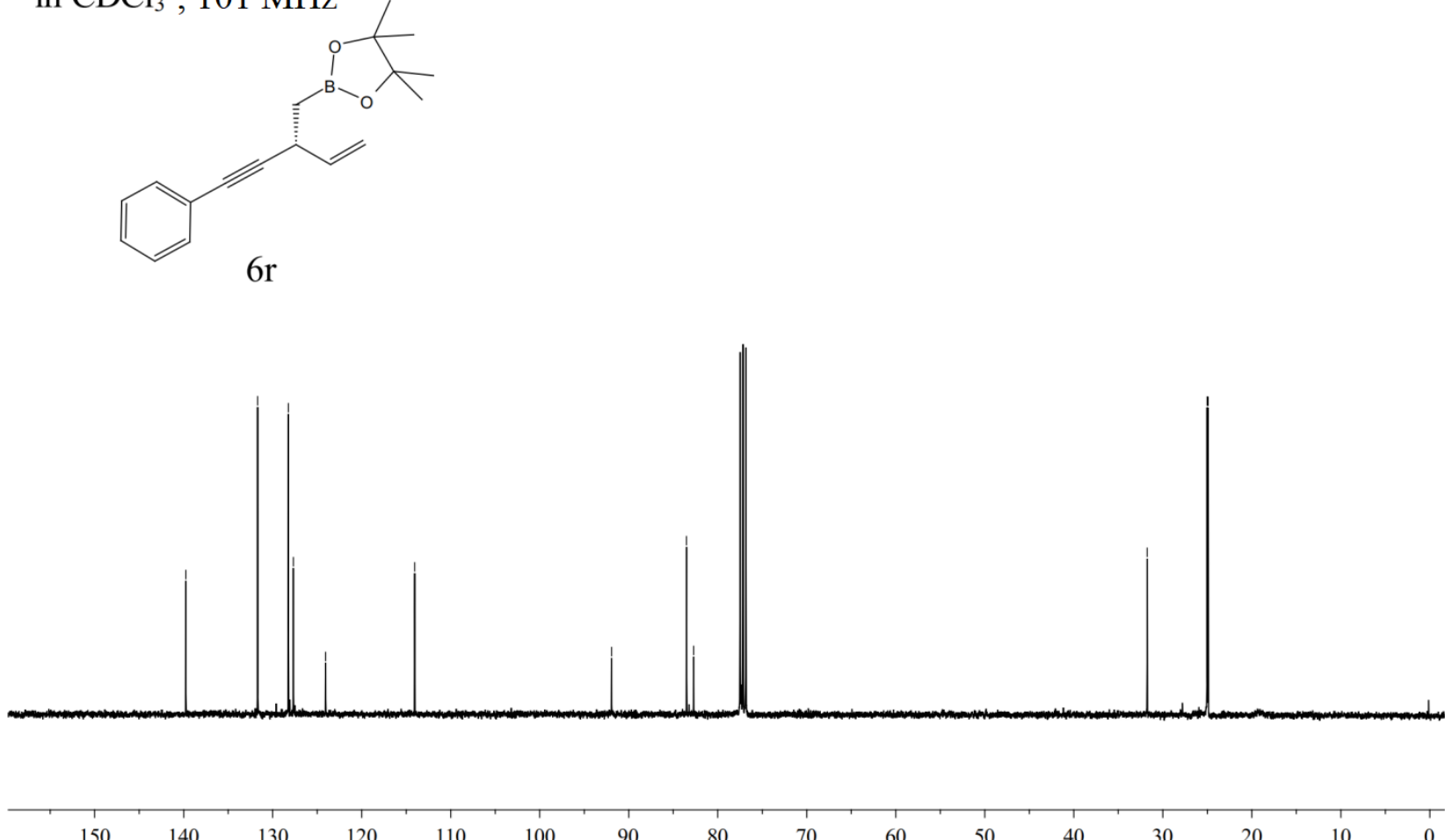


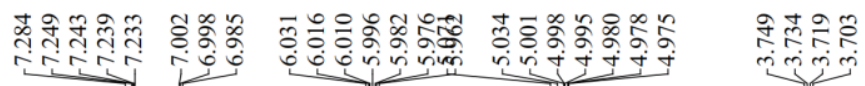

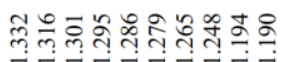

in $\mathrm{CDCl}_{3}, 400 \mathrm{MHz}$
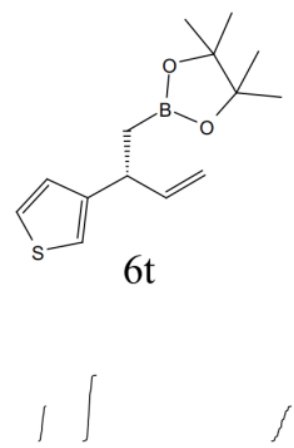

fs

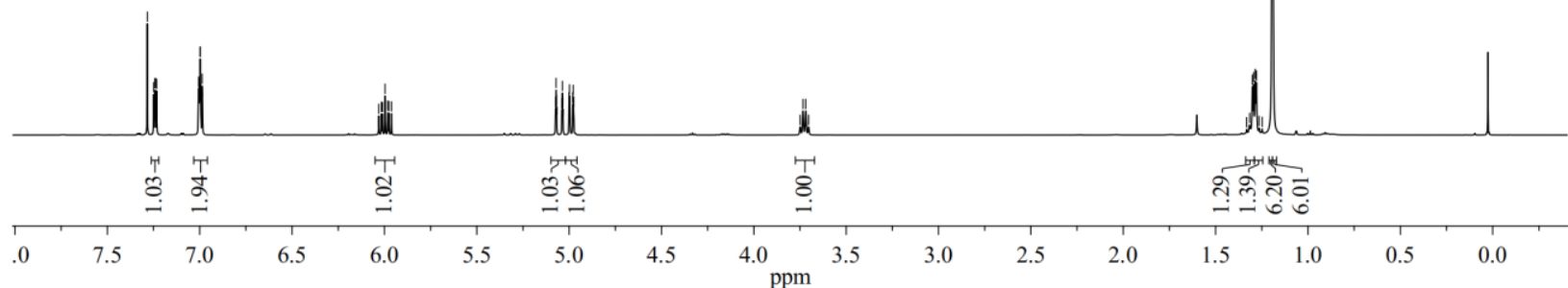

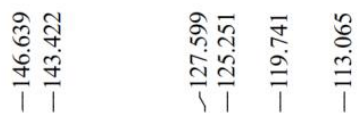

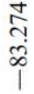

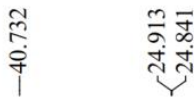

in $\mathrm{CDCl}_{3}, 101 \mathrm{MHz}$

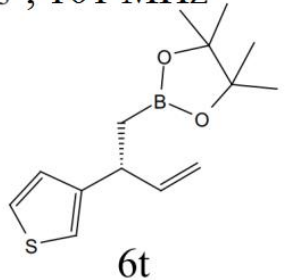

$6 \mathrm{t}$

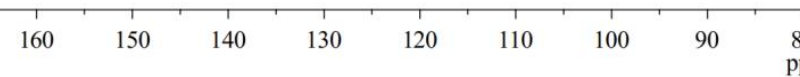



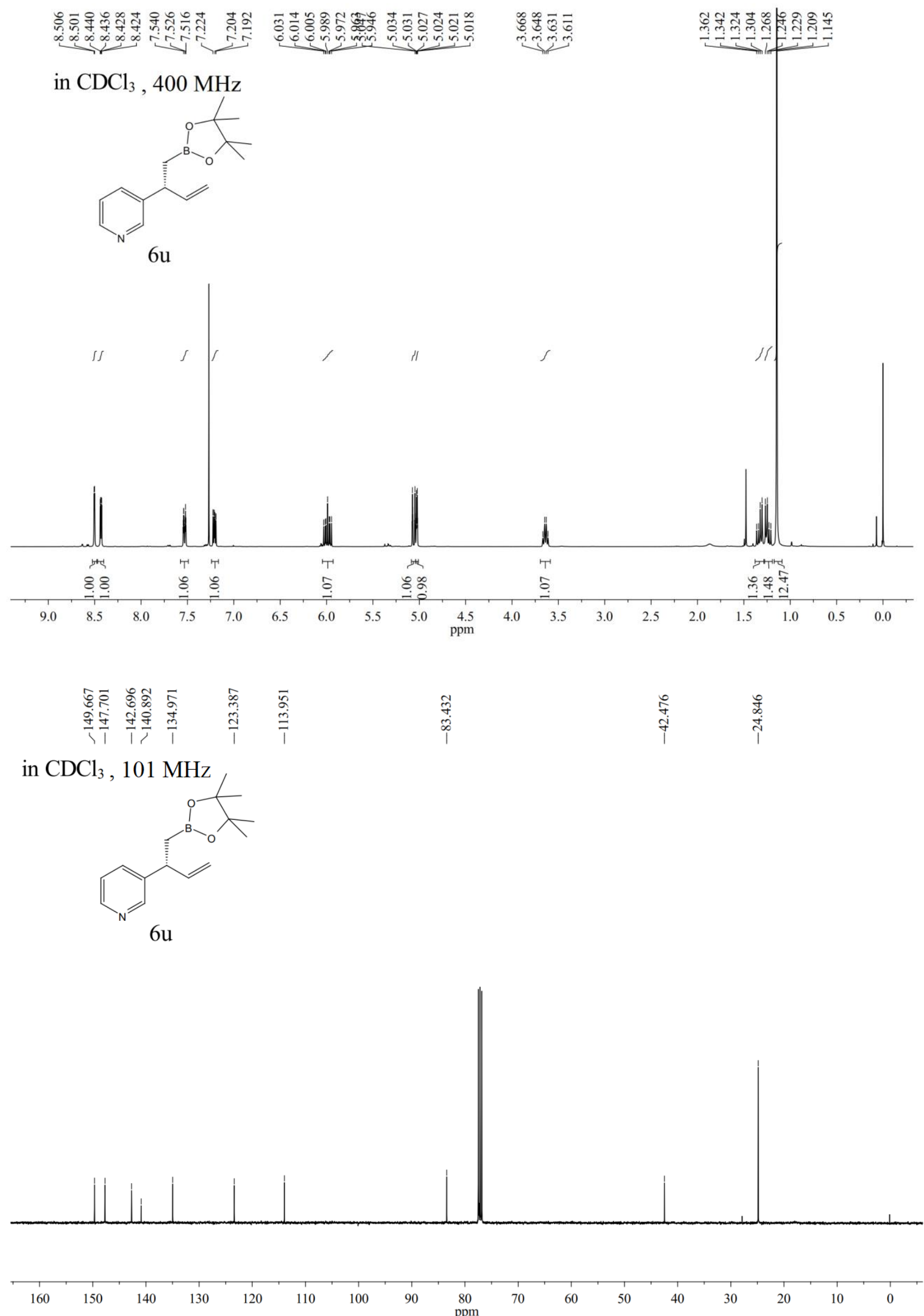


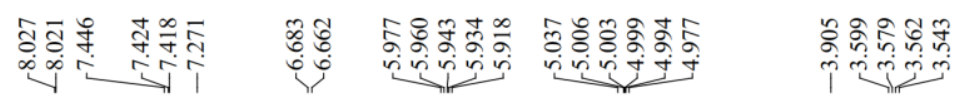

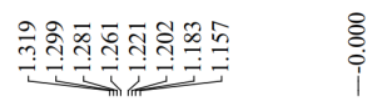

in $\mathrm{CDCl}_{3}, 400 \mathrm{MHz}$
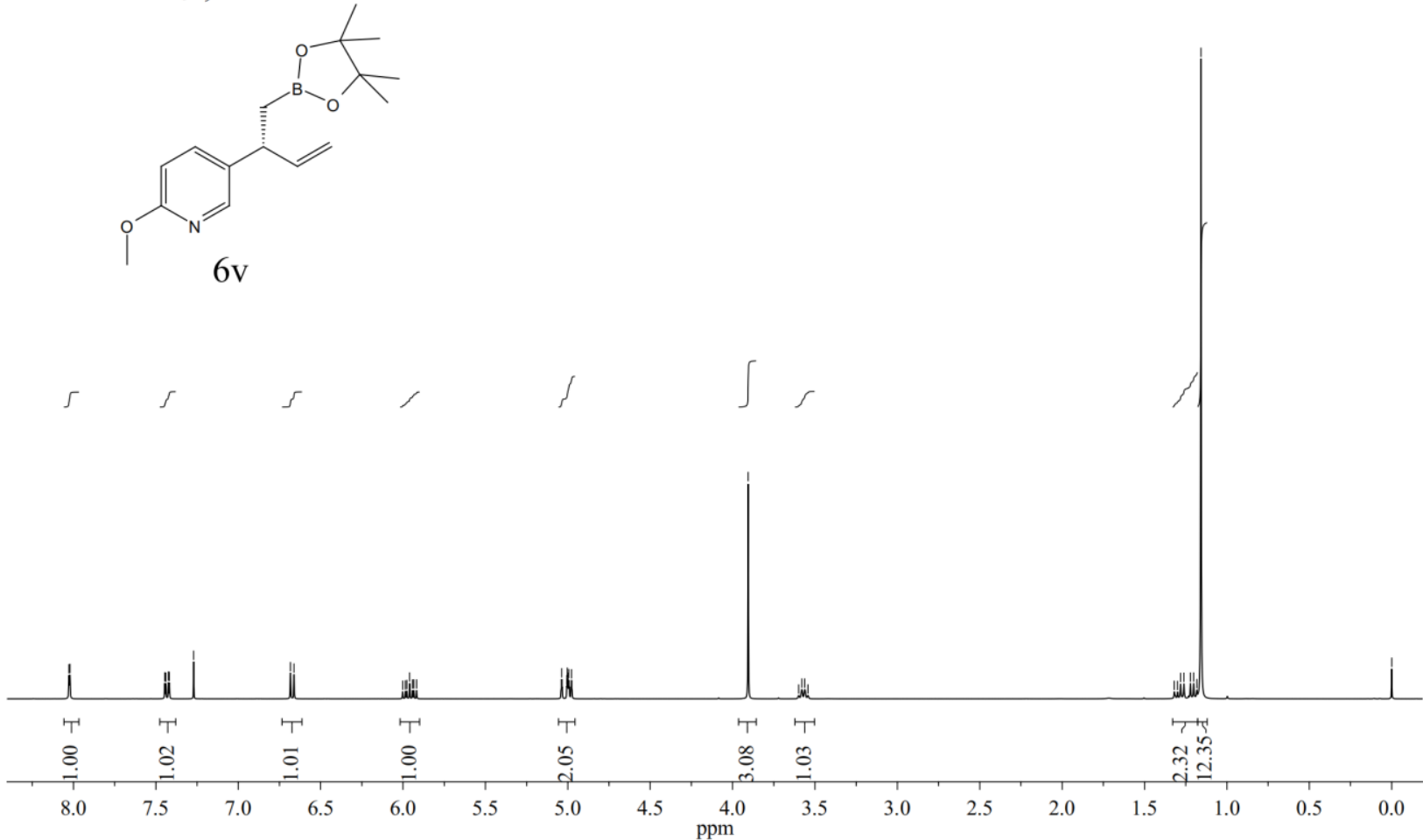

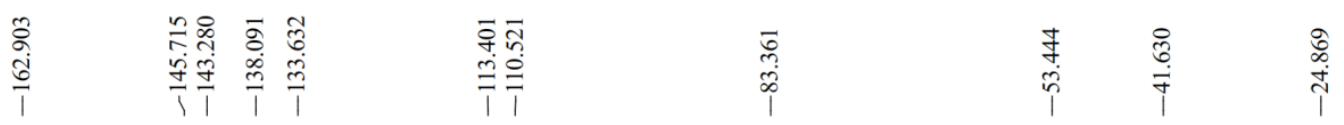

in $\mathrm{CDCl}_{3}, 101 \mathrm{MHz}$
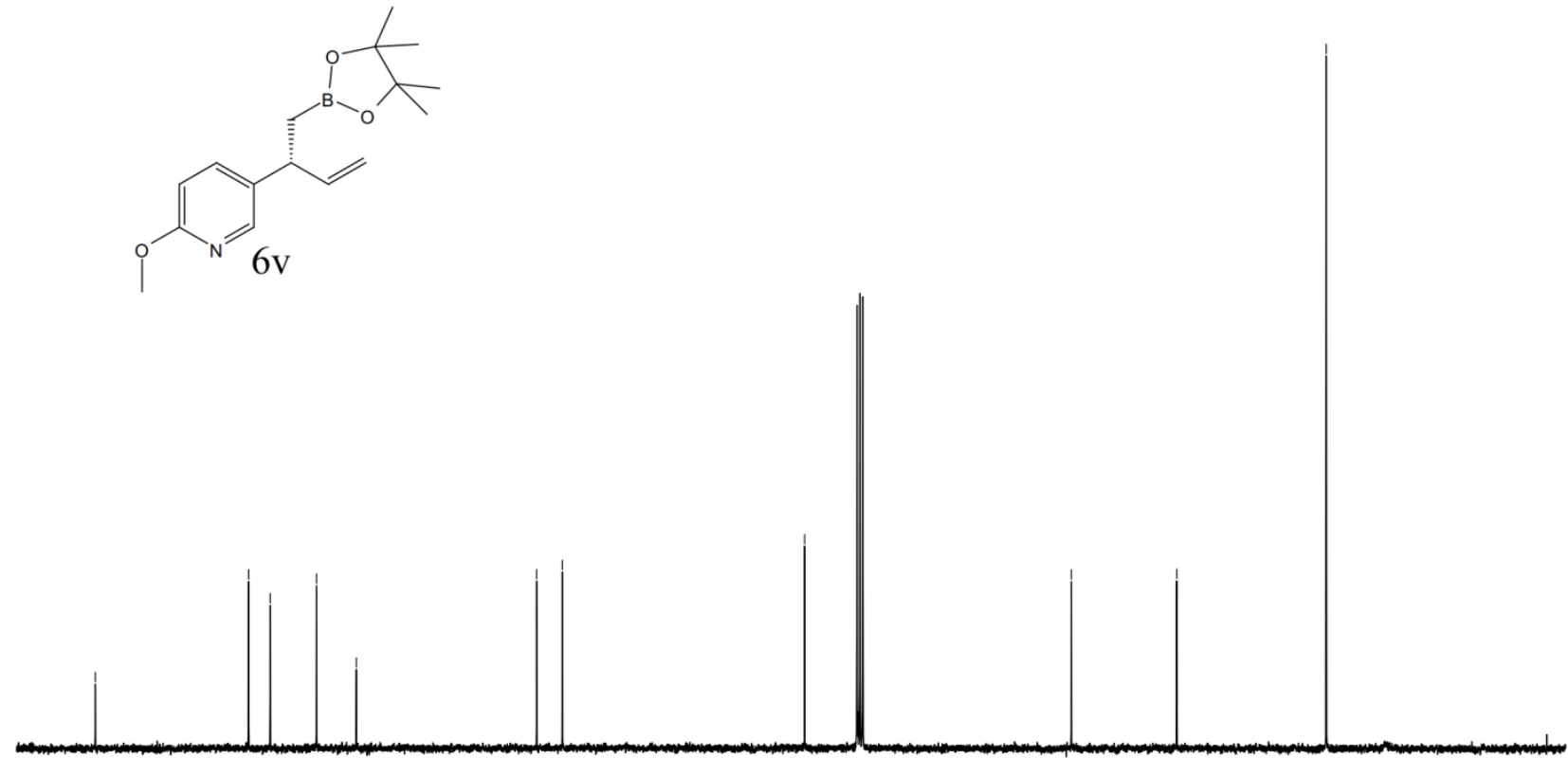

170

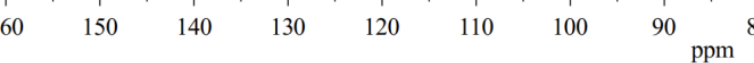



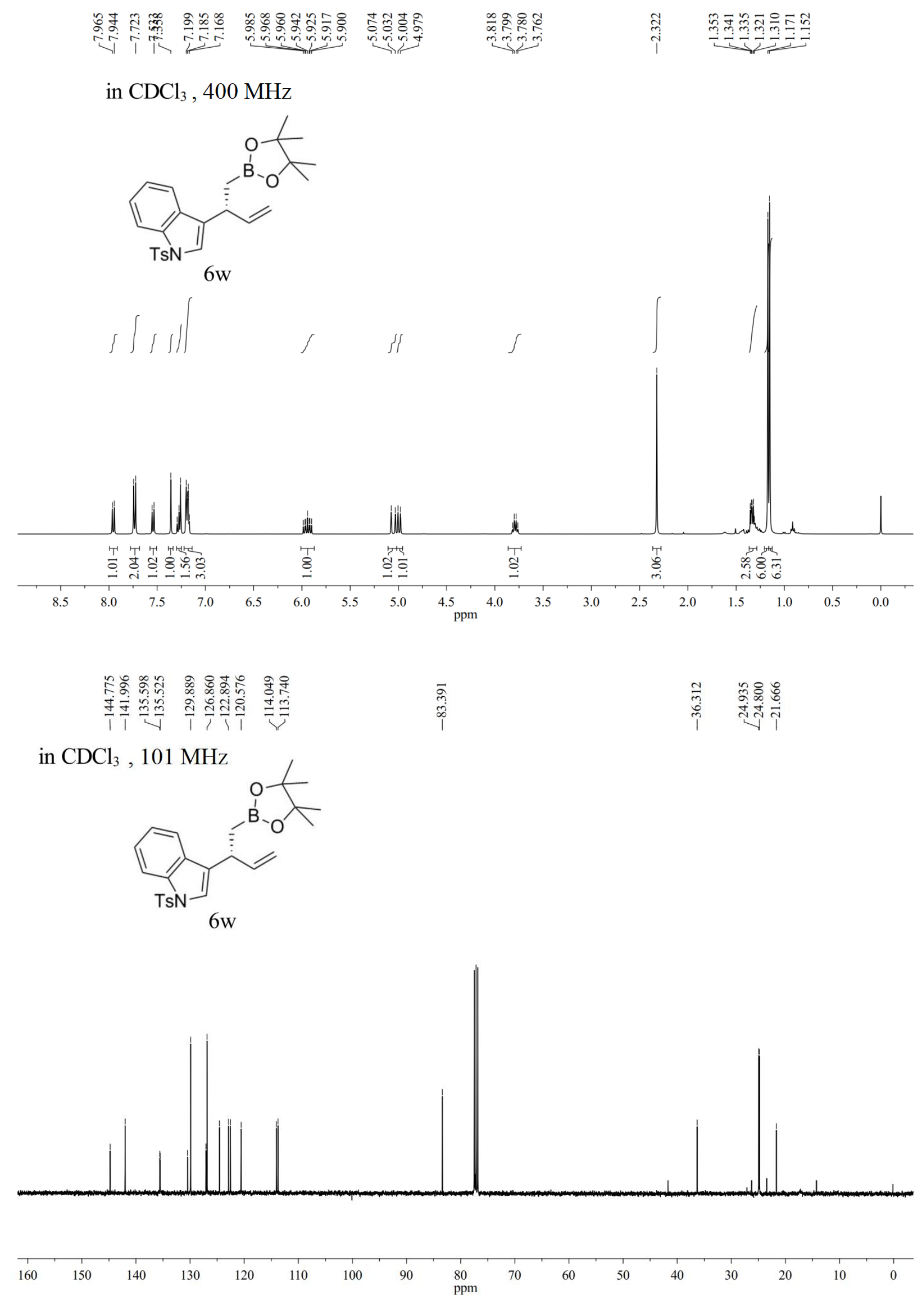

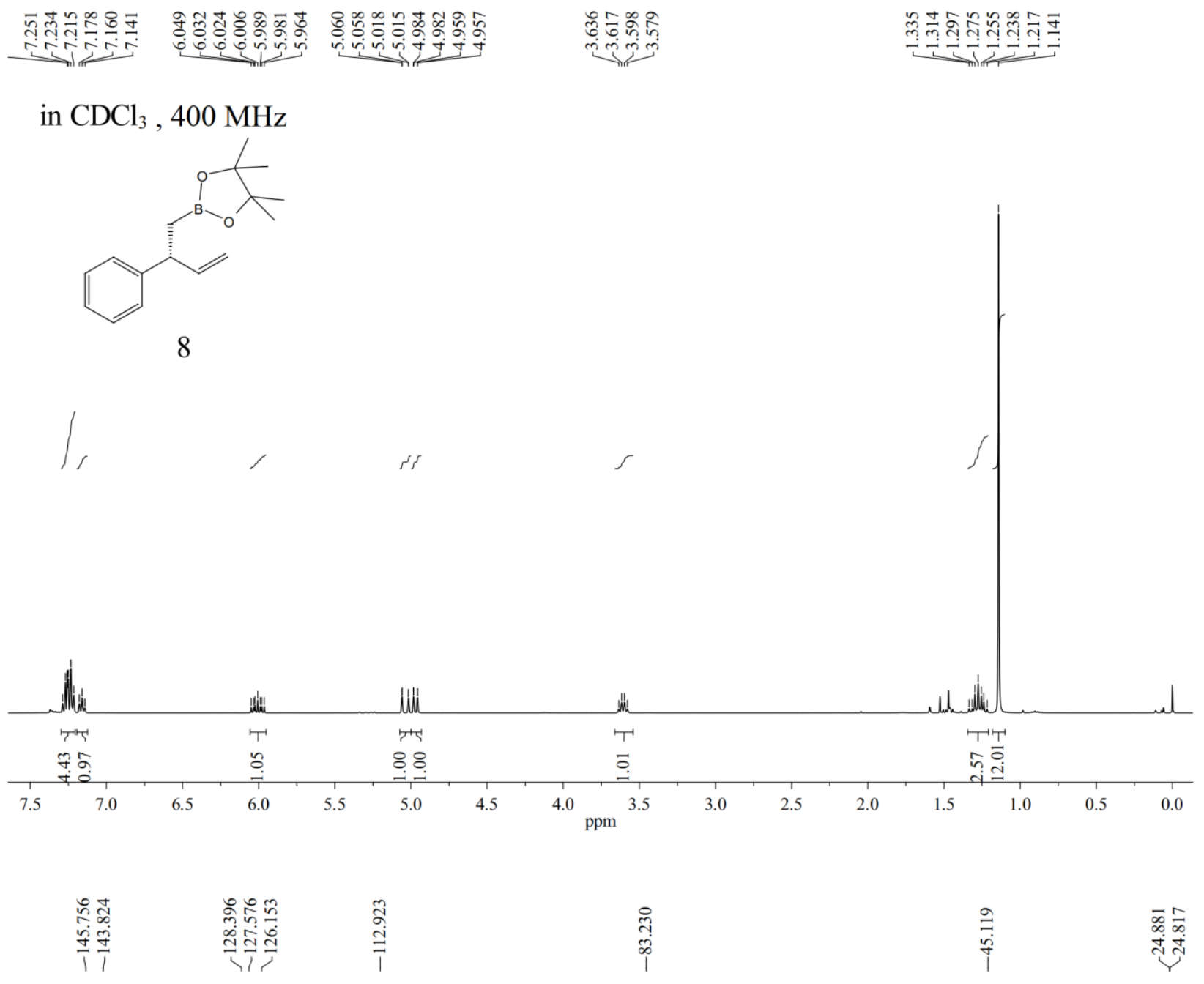

in $\mathrm{CDCl}_{3}, 101 \mathrm{MHz}$
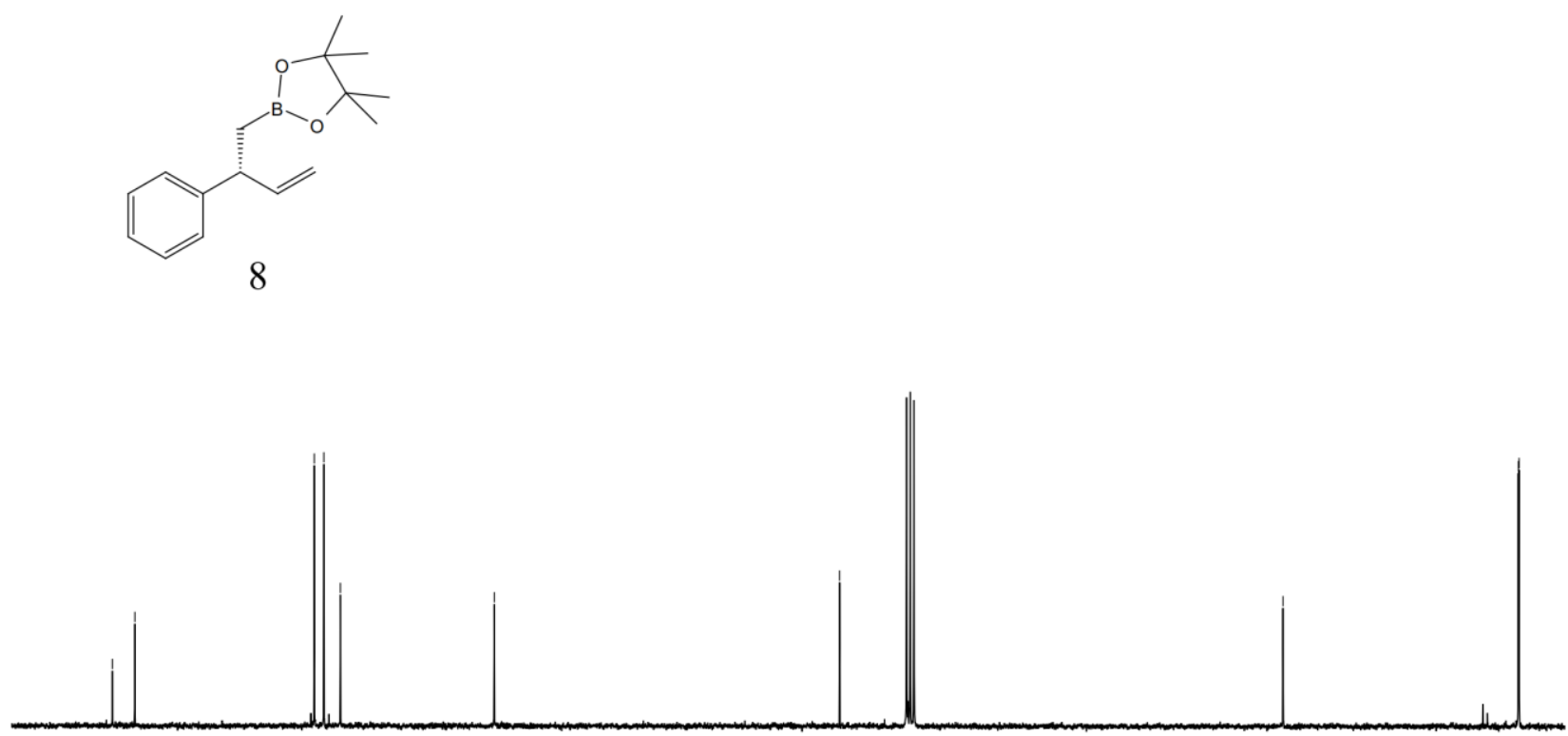

$\begin{array}{llllllllllllllllllllllllll}150 & 145 & 140 & 135 & 130 & 125 & 120 & 115 & 110 & 105 & 100 & 95 & \underset{\mathrm{ppm}}{90} & 80 & 75 & 70 & 65 & 60 & 55 & 50 & 45 & 40 & 35 & 30 & 25\end{array}$ 
in $\mathrm{CDCl}_{3}, 400 \mathrm{MHz}$<smiles>C=CC(CO)c1ccccc1</smiles>

9

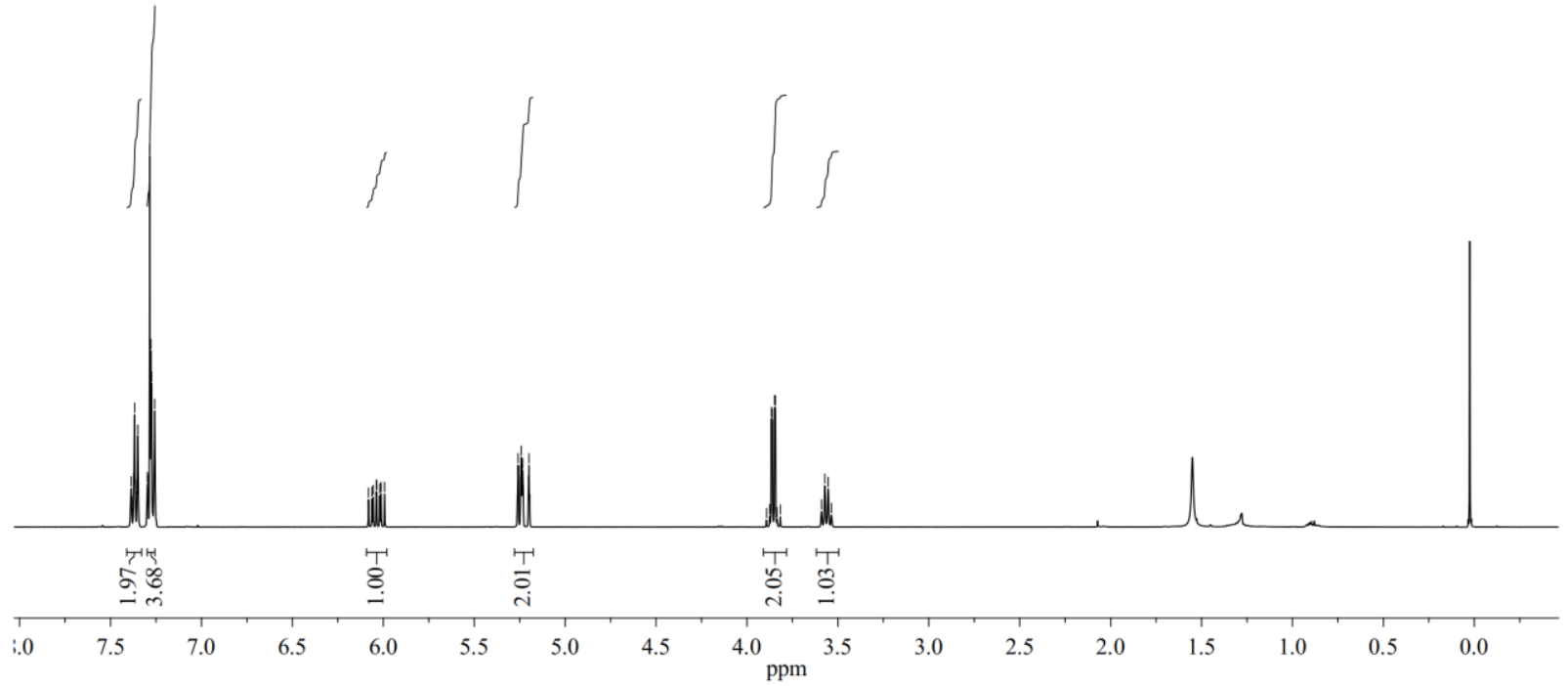

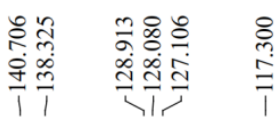

in $\mathrm{CDCl}_{3}, 101 \mathrm{MHz}$
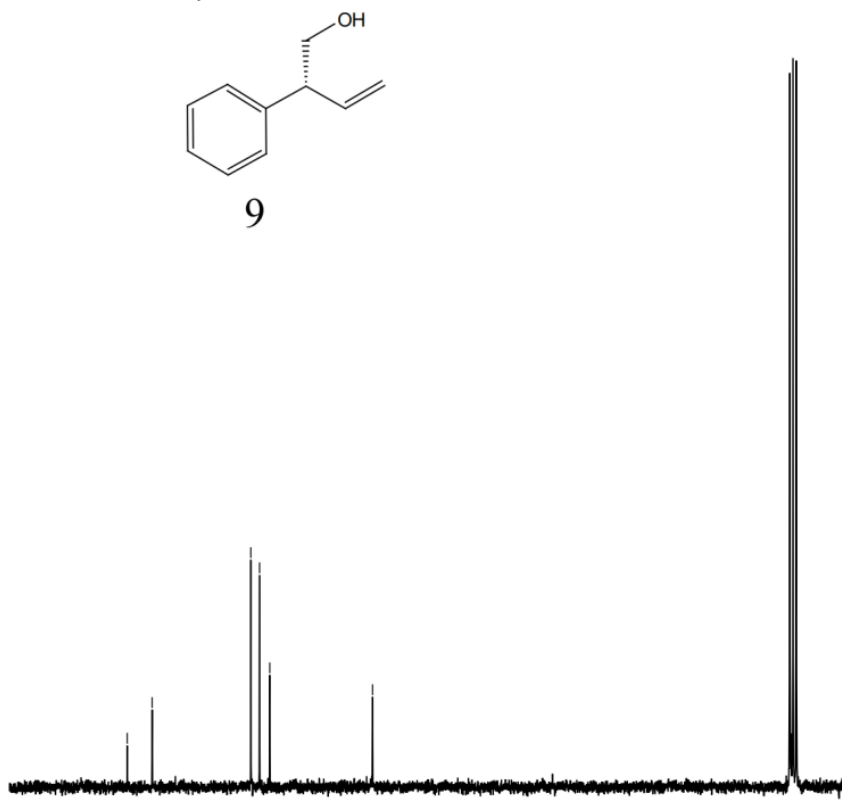

150
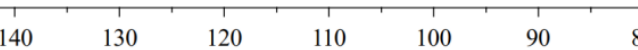

80 ppm 


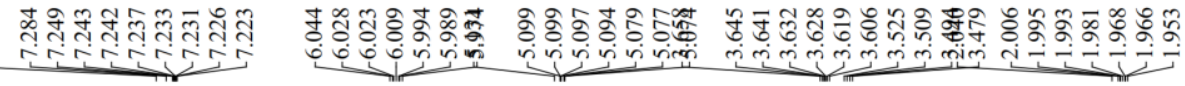

in $\mathrm{CDCl}_{3}, 400 \mathrm{MHz}$

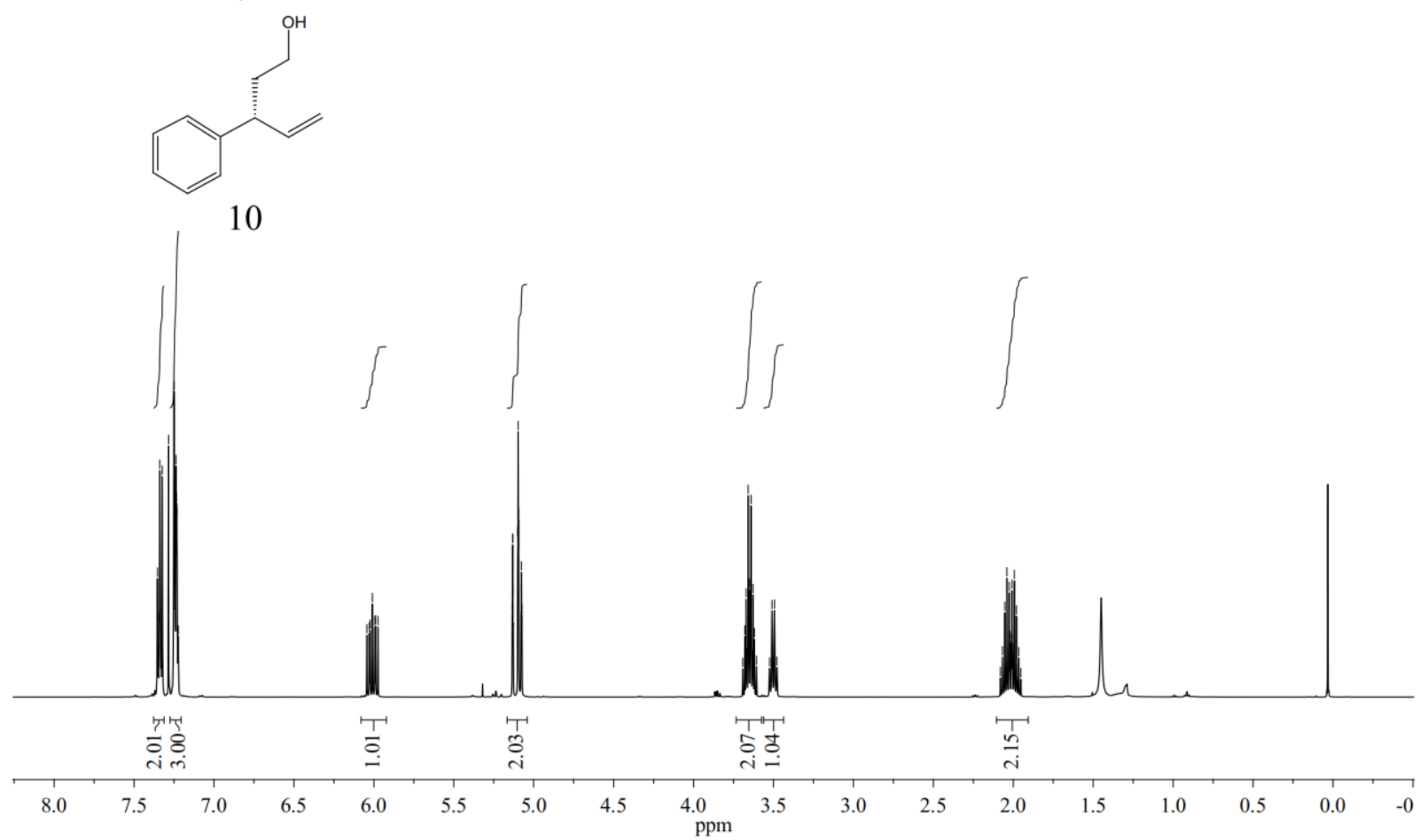

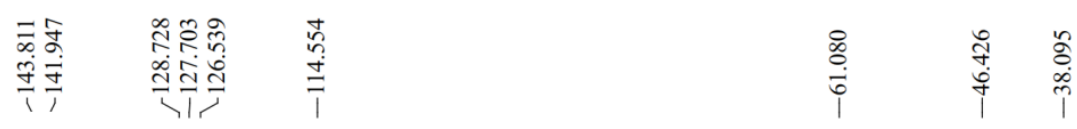

in $\mathrm{CDCl}_{3}, 101 \mathrm{MHz}$

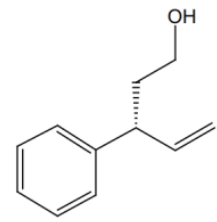

10

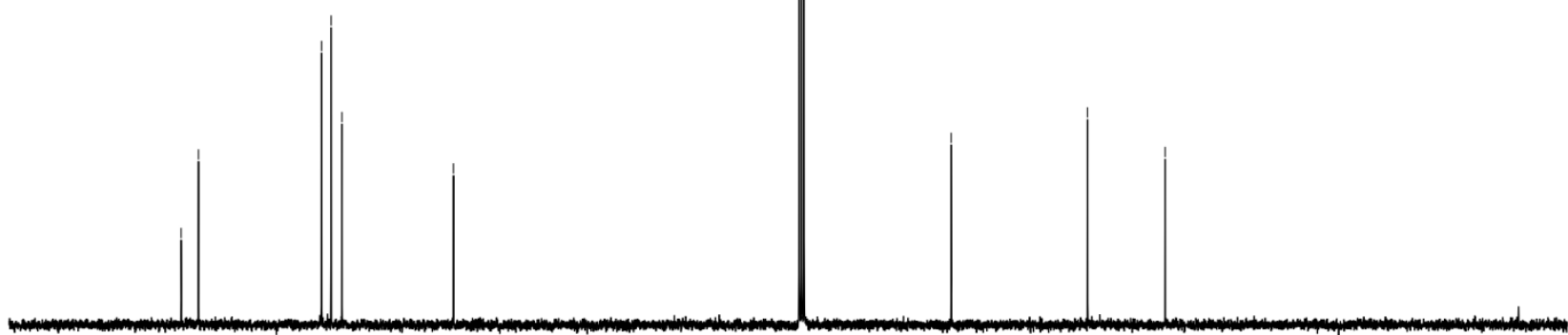

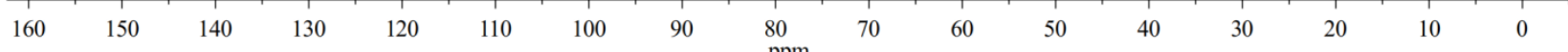


in $\mathrm{CDCl}_{3}, 400 \mathrm{MHz}$

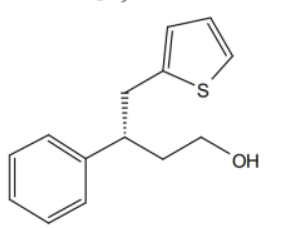

11
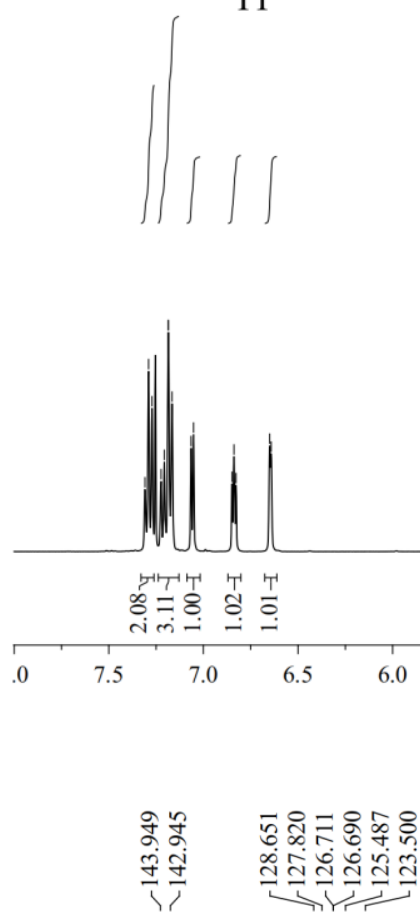

in $\mathrm{CDCl}_{3}, 101 \mathrm{MHz}$

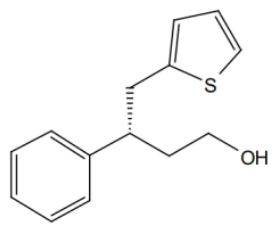

11

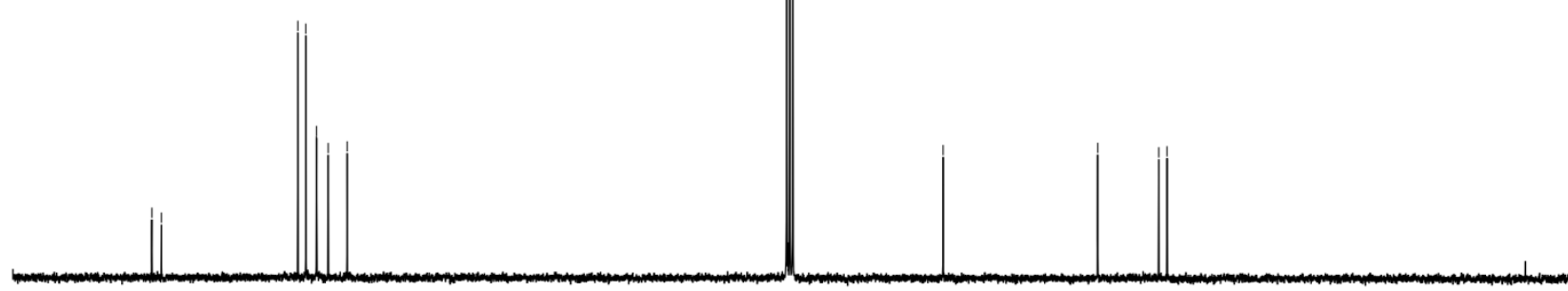

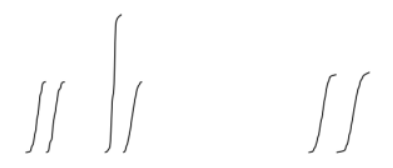

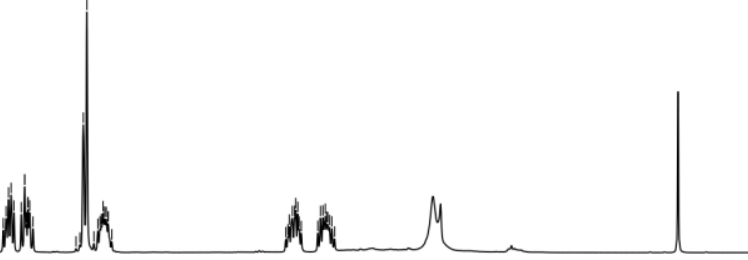

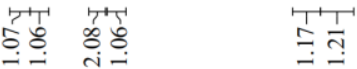

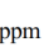

20

$\begin{array}{llll}1 & 1 & 1\end{array}$

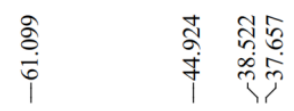

150
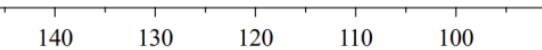

80 ppm 


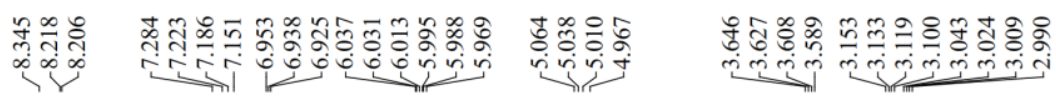

in $\mathrm{CDCl}_{3}, 400 \mathrm{MHz}$

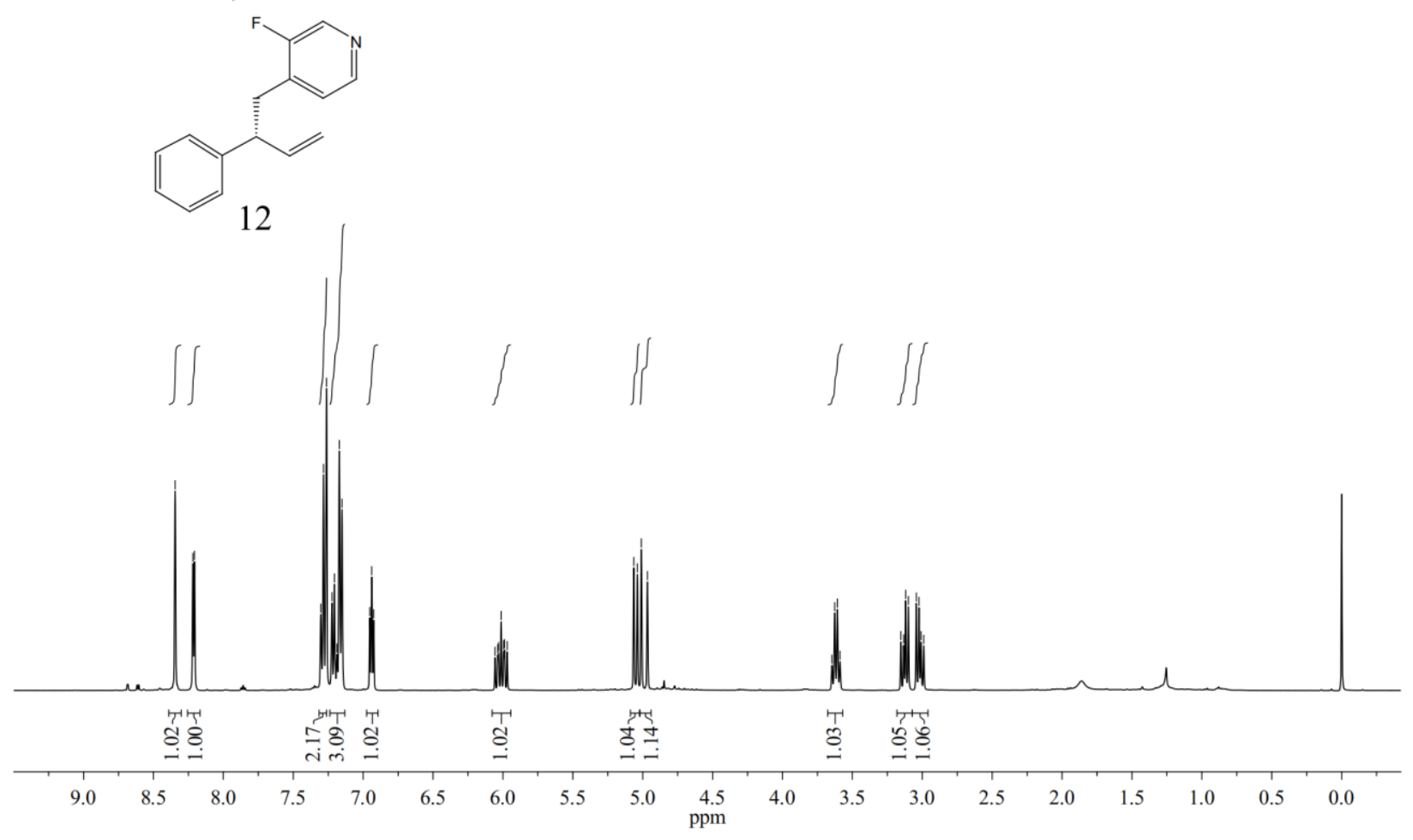

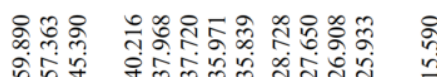

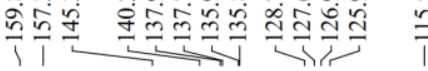

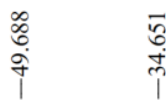

in $\mathrm{CDCl}_{3}, 101 \mathrm{MHz}$
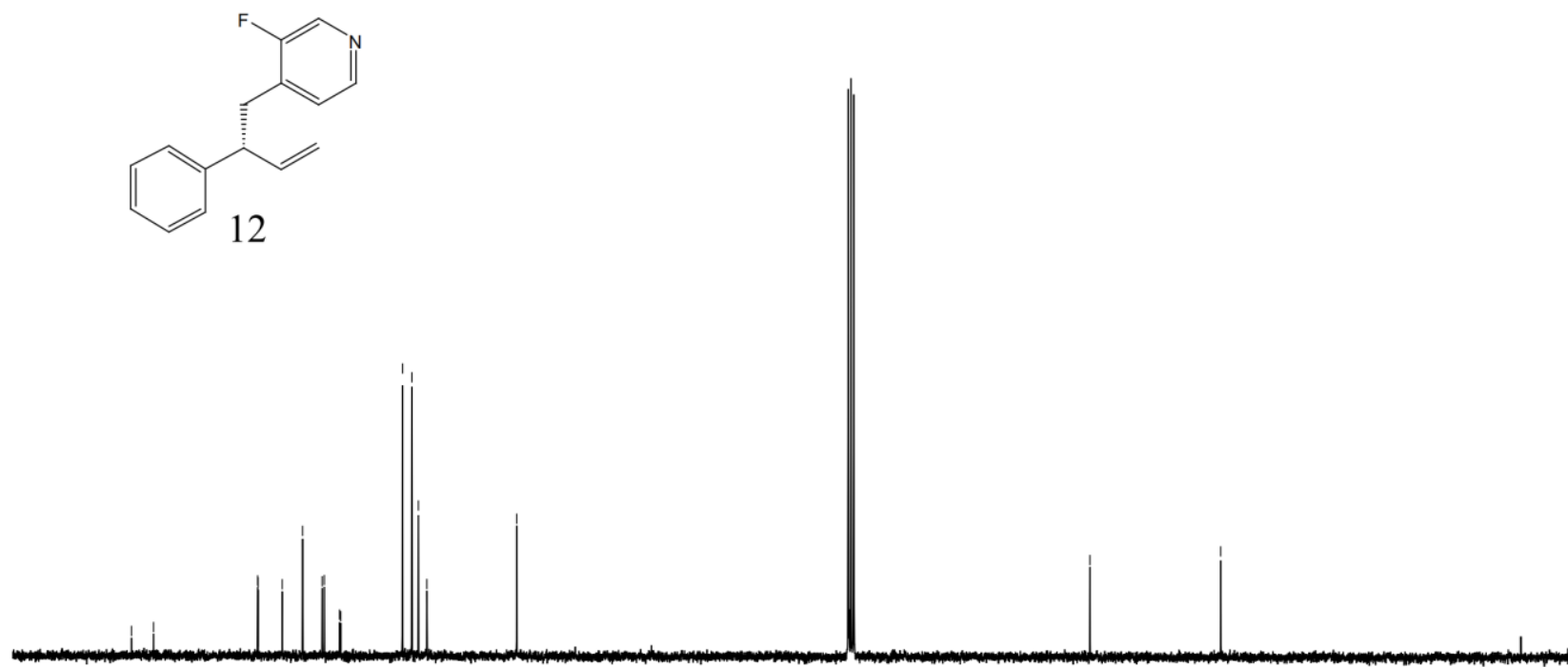

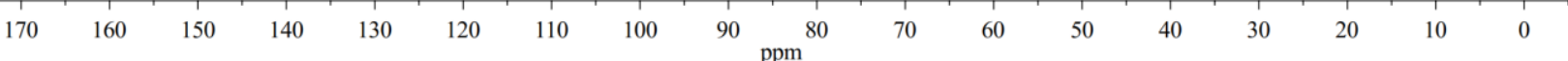

